Portland State University

PDXScholar

Winter 1-31-2017

\title{
Novel Compound, 84F2, Inhibits Calmodulin Deficient RyR2
}

Robert Carl Klipp

Portland State University

Follow this and additional works at: https://pdxscholar.library.pdx.edu/open_access_etds

Part of the Cardiology Commons, and the Physics Commons Let us know how access to this document benefits you.

Recommended Citation

Klipp, Robert Carl, "Novel Compound, 84F2, Inhibits Calmodulin Deficient RyR2" (2017). Dissertations and Theses. Paper 3484.

https://doi.org/10.15760/etd.5368

This Dissertation is brought to you for free and open access. It has been accepted for inclusion in Dissertations and Theses by an authorized administrator of PDXScholar. Please contact us if we can make this document more accessible: pdxscholar@pdx.edu. 
Novel Compound, 84F2, Inhibits Calmodulin Deficient RyR2

by

Robert Carl Klipp

A dissertation submitted in partial fulfillment of the requirements for the degree of

Doctor of Philosophy

in

Applied Physics

Dissertation Committee:

Jonathan Abramson, Chair

Steve Reichow

Robert Strongin

Drake Mitchell

Portland State University 2017 
(C) 2016 Robert Carl Klipp 


\section{Abstract}

The cardiac ryanodine receptor (RyR2) plays a key role in excitation-contraction coupling (ECC). Mutations in RyR2 are known to be linked to the arrhythmogenic disorder, catecholaminergic polymorphic ventricular tachycardia (CPVT), a deadly disease which is characterized by a leak of calcium from sarcoplasmic reticulum and a decrease in calmodulin (CaM) binding. A novel drug, 84F2, shown to inhibit arrhythmias in RyR2R176Q heterozygous CPVT mouse hearts $(2.5 \mu \mathrm{g} / \mathrm{kg})$, decrease spark frequency in cells derived from CPVT mice $\left(\mathrm{IC}_{50}=35 \mathrm{nM}\right)$, and inhibit RyR2 single channel activity at low nanomolar concentrations ( $\left.\mathrm{IC}_{50}=8 \mathrm{nM}\right)$. When CaM is added back to RyR2, 84F2's ability to inhibit channel activity is suppressed approximately 250 fold. A metabolite of $84 \mathrm{~F} 2,78 \mathrm{~F} 3$, is shown to also be active in the inhibition of RyR2. We propose that 84F2 decreases arrhythmias by binding to the CaM deficient RyR2, but does not affect normal ECC when CaM is present. This work characterizes for the first time a class of drugs whose inhibitory affects are dependent upon the removal of CaM from RyR2. 


\section{Dedication}

This work is dedicated to my father, Ross Klipp, and mother, Colleen Klipp. Their hard work, support and encouragement have allowed me to pursue my own dreams. 


\section{Acknowledgements}

I would first like to sincerely thank my advisor, Jonathan Abramson. He took me into his lab and has supported me through this work and my time as a graduate student. His willingness and desire to help, no matter when I asked, was a huge contribution in my success as a graduate student and the completion of this work.

I would like to thank the Department of Physics and ELEX Biotech for their financial assistance in my time as a graduate student.

I would like to thank my committee members for their time in reviewing this work.

Finally, I would like to thank members of the Abramson Lab, who have helped me through the years: Tom Dornan, Yanping Ye, Laura Owen, and Merve Tekmen-Clark. 


\section{Table of Contents}

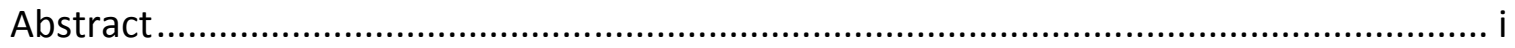

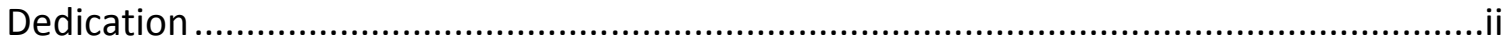

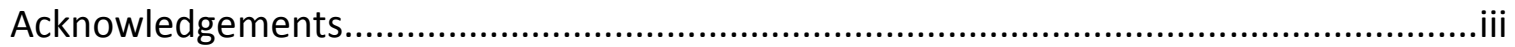

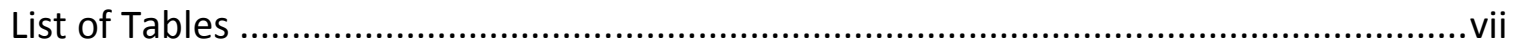

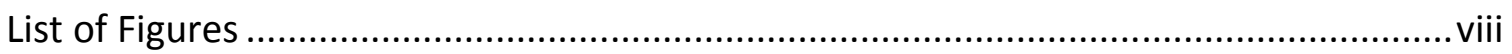

1 Introduction and Review of Related Literature .................................................. 1

$1.1 \quad$ Excitation - Contraction Coupling …......................................................... 1

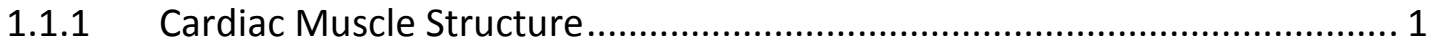

1.1.2 The Action Potential ..................................................................................... 4

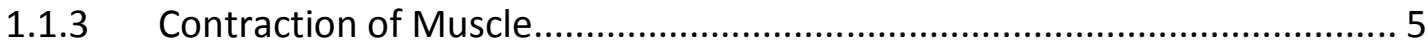

1.2 Calcium-Induced Calcium Release ............................................................... 5

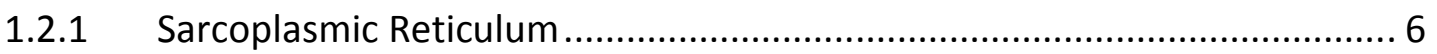

1.3 The SR Ca ${ }^{2+}$-Release Channel: The Ryanodine Receptor ................................. 7

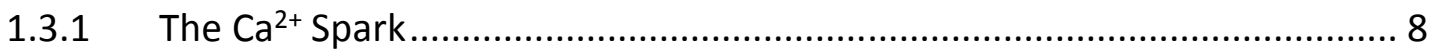

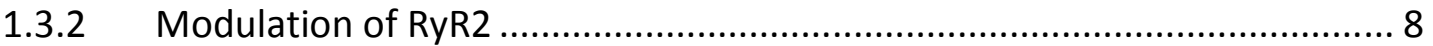

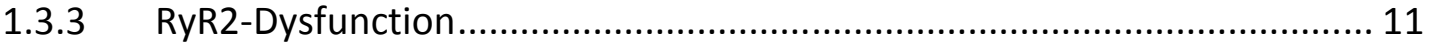

1.4 Catecholaminergic Polymorphic Ventricular Tachycardia (CPVT) ................... 12

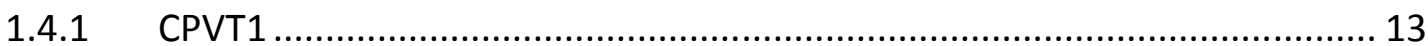

1.4.2 CPVT Pathway Leading to RyR2-Dysfunction......................................... 14

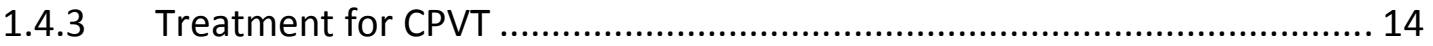

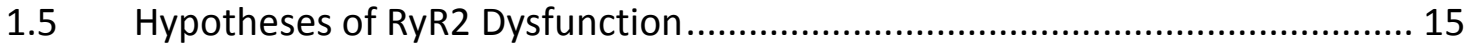

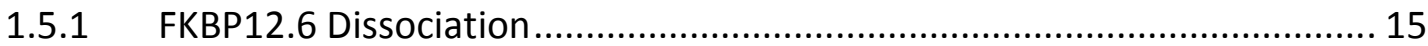

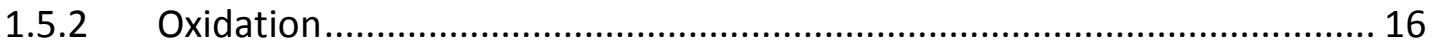

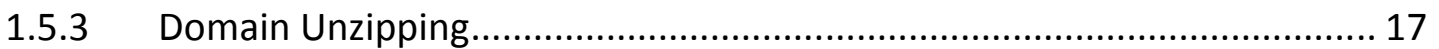

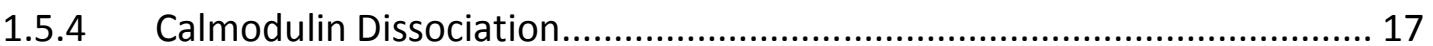

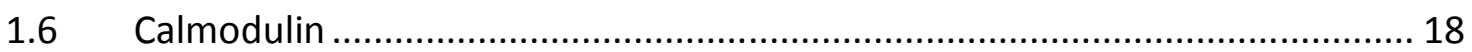

1.6.1 Calmodulin Interaction with the Ryanodine Receptor ............................ 20

1.6.1.1 Calmodulin Binding to the Ryanodine Receptor .............................. 20 
1.6.1.2 Calmodulin Modulation of the Ryanodine Receptor .......................... 20

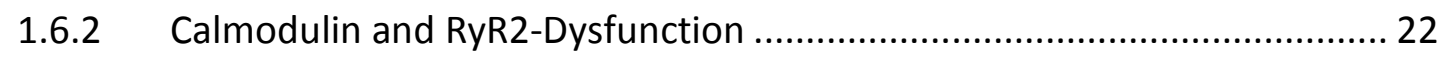

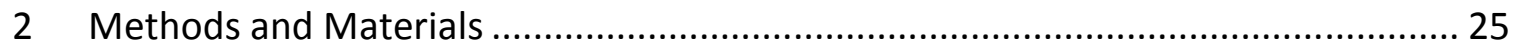

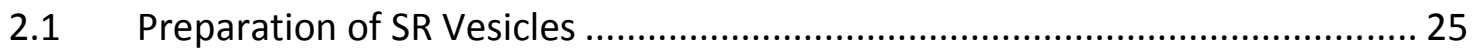

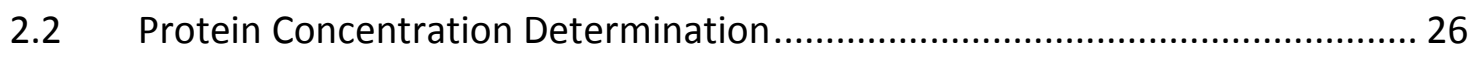

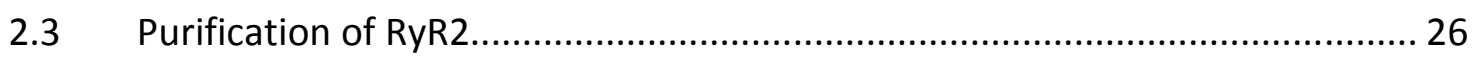

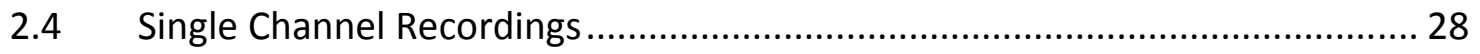

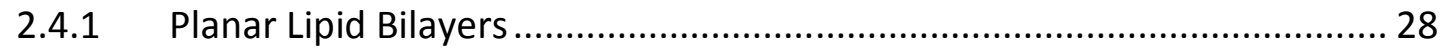

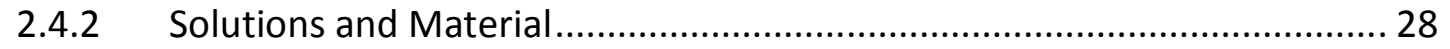

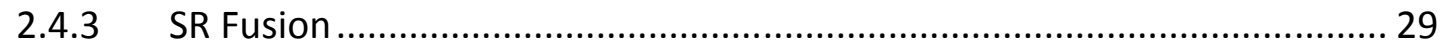

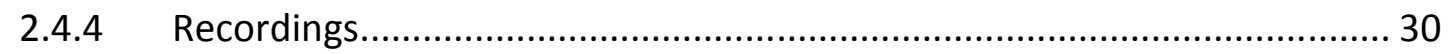

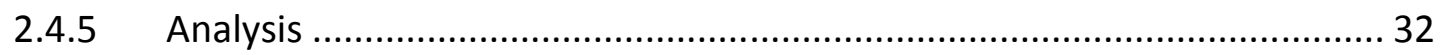

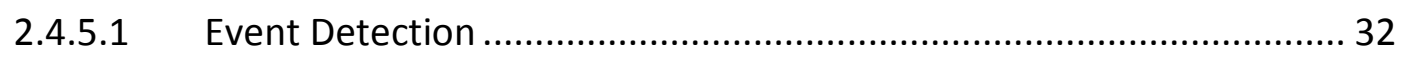

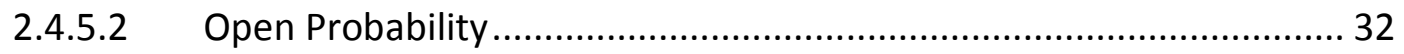

2.4.5.3 Open and Closed Dwell Times ......................................................... 33

2.4.5.4 Determination of Statistical Significance of Dwell Times................... 33

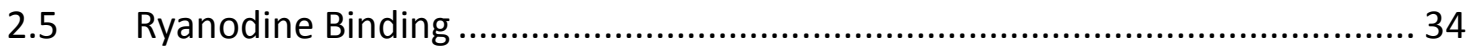

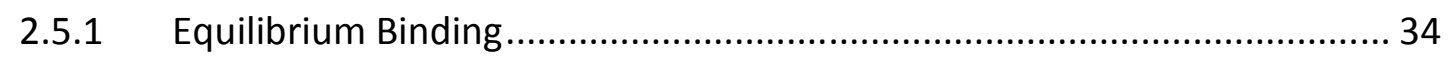

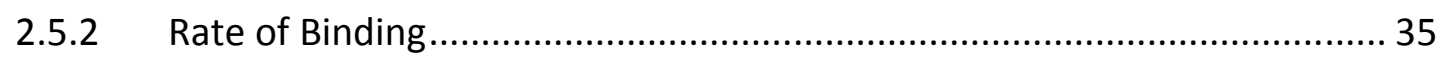

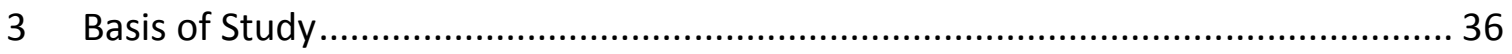

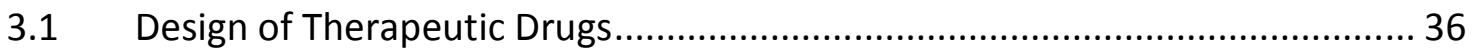

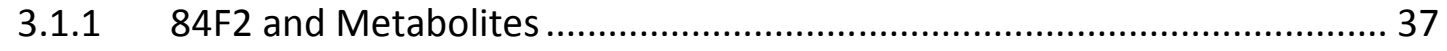

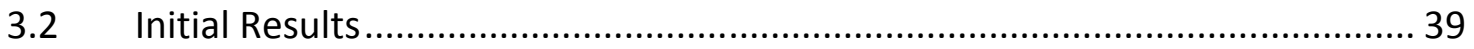

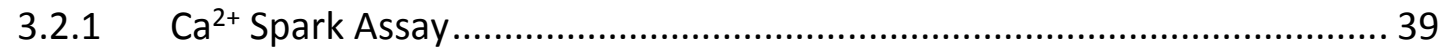

3.2.2 Monitoring Arrhythmia at Whole Animal Level.................................... 43

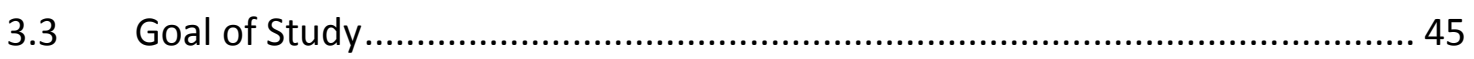

4 Results: Determination if 84F2 Directly Inhibits Purified RyR2 ............................. 46

4.1 84F2 Inhibition of Purified RyR2 at the Single Channel Level.......................... 47

4.2 Effect of 84F2 on Purified RyR2 Dwell Times................................................ 51

4.3 Ionic Strength Dependence of 84F2 Inhibition of Purified RyR2 .................... 54

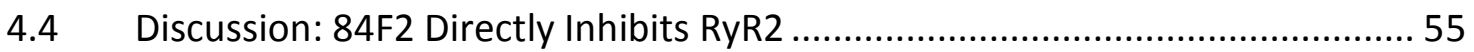


5 Results: Determination of the Active Metabolite of 84F2

5.12 -(Diethylamino)ethanol (DEAE) Inhibition of Purified RyR2 at the Single Channel Level 58

$5.278 F 3$ Inhibition of Purified RyR2 at the Single Channel Level...........................61

5.3 Discussion: Following Breakdown of 84F2, 78F3 Inhibits RyR2 ..................... 64

6 Results: Determination if 84F2 Inhibits RyR2 from Crude Cardiac SR ...................... 67

6.1 84F2 Single Channel Inhibition of RyR2 from Crude Cardiac SR...................... 68

6.2 84F2, 78F3, and DEAE Inhibition of Equilibrium ${ }^{3} \mathrm{H}$-Ryanodine Binding to RyR2

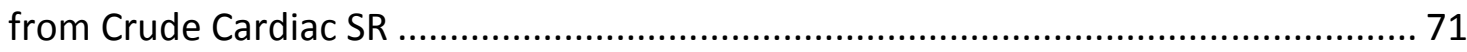

6.3 Rate of ${ }^{3} \mathrm{H}$-Ryanodine Binding in the Presence of 84F2 ............................... 73

6.4 Discussion: 84F2 has Reduced Inhibition of RyR2 from Crude Cardiac SR ...... 75

7 Results: Determination of Calmodulin's Role in 84F2's Inhibition of RyR2 ............. 79

7.1 Inhibition of Purified RyR2 Single Channel Activity by 84F2 in the Presence of

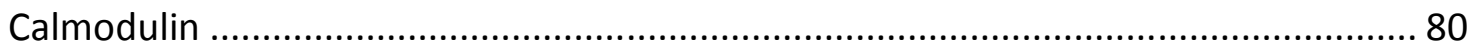

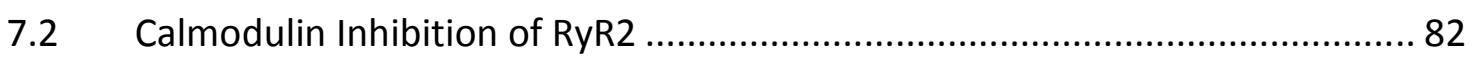

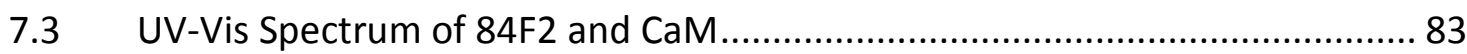

7.4 Discussion: CaM Reduces 84F2's Potency in Inhibiting Purified RyR2 …......... 85

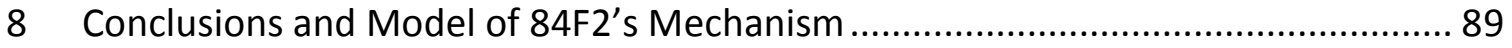

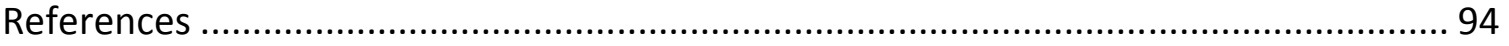




\section{List of Tables}

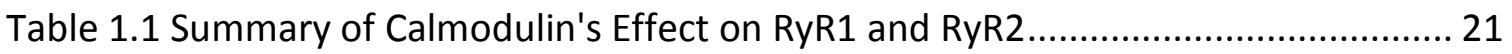

Table 4.1 Summary of 84F2 Inhibition of RyR2 …...................................................... 55

Table 5.1 Summary of 84F2, 78F3, and DEAE Inhibition of Purified RyR2 ....................... 64

Table 6.1 Summary of 84F2 Inhibition in the rate of ${ }^{3} \mathrm{H}$-Ryanodine Binding .................. 73

Table 6.2 Summary of 84F2 Inhibition in the Presence of Associated Proteins............... 75

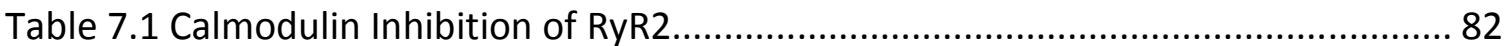

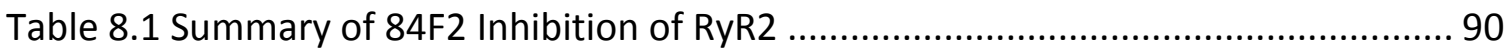




\section{List of Figures}

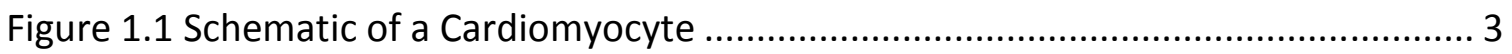

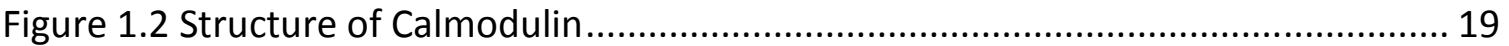

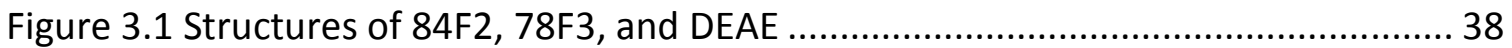

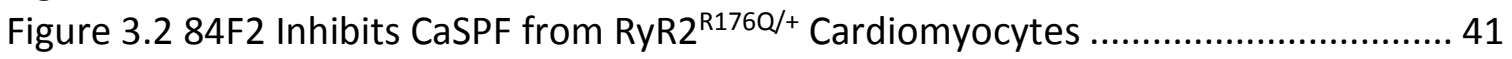

Figure 3.3 78F3 Inhibits CaSPF from RyR2R176Q/+ Cardiomyocytes............................ 42

Figure 3.4 84F2 Suppresses the Induction of Ventricular Tachycardia (VT) in RyR2 ${ }^{\mathrm{R} 1760 /+}$

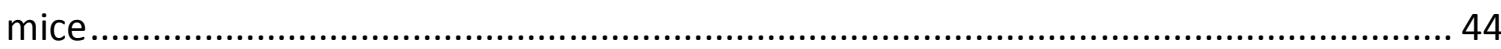

Figure 4.1 Purified RyR2 Single Channel Traces in the Presence of 84F2 ....................... 49

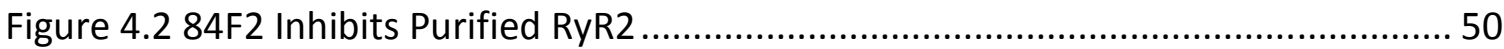

Figure 4.3 84F2 Increases the Closed Dwell Time of Purified RyR2 ............................... 52

Figure 4.4 84F2 Decreases the Open Dwell Time of Purified RyR2 at High Concentrations

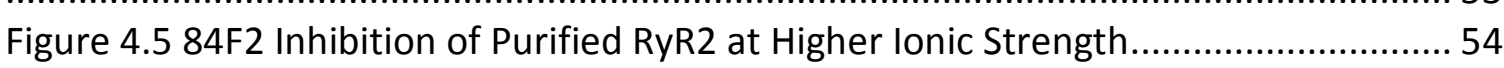

Figure 5.1 Purified RyR2 Single Channel Traces in the Presence of DEAE...................... 59

Figure 5.2 DEAE Does Not Inhibit Purified RyR2 ...................................................... 60

Figure 5.3 Purified RyR2 Single Channel Traces in the Presence of 78F3.........................62

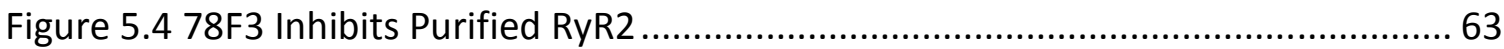

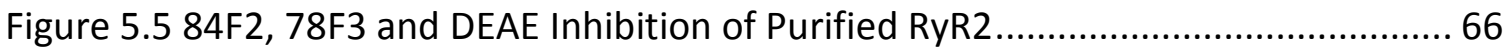

Figure 6.1 Non-Purified RyR2 Single Channel Traces in the Presence of 84F2 ............... 69

Figure 6.2 84F2 Shows Diminished Inhibition of RyR2 from Crude Cardiac SR .............. 70

Figure 6.3 Equilibrium ${ }^{3} \mathrm{H}$-Ryanodine Binding in the Presence of 84F2, 78F3, and DEAE 72

Figure 6.4 84F2 Decreases the Rate of ${ }^{3} \mathrm{H}$-Ryanodine Binding to RyR2 from Crude Cardiac

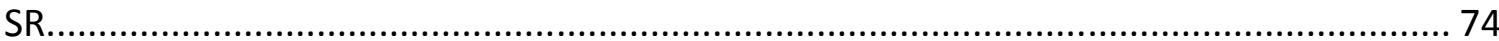

Figure 6.5 Comparison of Single Channel Inhibition of RyR2 from Crude Cardiac SR and

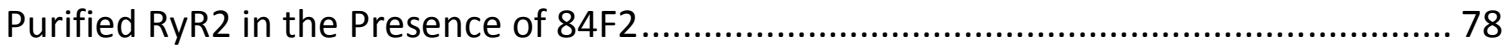

Figure 7.1 Inhibition of Purified RyR2 by 84F2 in the Presence of Calmodulin ............... 81

Figure 7.2 UV-Vis Spectrums of 84F2 and Calmodulin ............................................ 84

Figure 7.3 Calmodulin Decreases Inhibition of Purified RyR2 by 84F2 2........................ 88

Figure 8.1 Comparison of RyR2 Single Channel Activity by 84F2 ............................... 91

Figure 8.2 Model of 84F2 Inhibition of Arrhythmia resulting from RyR2 ${ }^{\mathrm{R} 176 \mathrm{Q} /+} \ldots \ldots \ldots \ldots . . . . . .93$ 


\section{Introduction and Review of Related Literature}

The contraction of muscle is responsible for nearly every facet of day-to-day life. The heart, like any other muscle, relies upon the basic principle of muscle contraction. When this fundamental process is disturbed, life can be drastically altered, or taken away.

Here we discuss the fundamentals of healthy cardiac muscle contraction, reviewing the structure of contractile elements and how excitation-contraction coupling regulates these structures. We highlight the importance of $\mathrm{Ca}^{2+}$ in the process of excitationcontraction coupling and how mishandling of $\mathrm{Ca}^{2+}$ leads to improper cardiac function, resulting in arrhythmia, heart failure, and death. One source of $\mathrm{Ca}^{2+}$ mishandling, dysfunction in the cardiac ryanodine receptor (RyR2), will be examined in its relation to a genetic arrhythmogenic disorder, CPVT.

\subsection{Excitation - Contraction Coupling}

The process by which electrical signals generated in the nervous system propagate and lead to contraction of muscle is referred to as excitation-contraction coupling (ECC). Both the skeletal and cardiac muscle system undergo ECC, but their mechanism of gating are different. Details of ECC in skeletal muscle will not be discussed in this work, except to highlight key differences.

\subsubsection{Cardiac Muscle Structure}

The heart is separated into 2 major regions. The region where blood enters the heart, the left and right atrium, and the region where blood is pumped to the rest of the body, 
the left and right ventricles. Both of these regions are composed of muscle fibers made up of cardiac muscle cells, or cardiomyocytes.

Contractile cardiomyocytes in the ventricle are composed of groupings myofibrils. These myofibrils are formed from long chain of sarcomeres. This sarcomere is the contractile element of the muscle fiber.

Thin filaments, composed primarily of actin, overlap with thick filaments, composed primarily of myosin, to make up each sarcomere. These areas of overlap, known as Abands, give muscle its characteristic striated pattern. The area containing the thin filament-actin, known as the I-band, is anchored at an area known as the Z-disk. Each sarcomere spans between two Z-disks.

Surrounding each cardiomyocyte is a cellular membrane known as the sarcolemma. At the Z-disks, the sarcolemma invaginates into the cardiomyocyte. These invaginations are known as transverse, or T-tubules.

In a similar manner, myofibrils are surrounded by a web-like membrane system, known as the sarcoplasmic reticulum (SR). Terminal cisternae (TC), which are enlarged regions of SR, meet up with the T-tubules to form a junction. A key difference between cardiac and skeletal muscle is that cardiac muscle forms a dyadic junction with one T-tubule and one TC, while skeletal muscle forms a triadic junction, with two TC per T-tubule. 


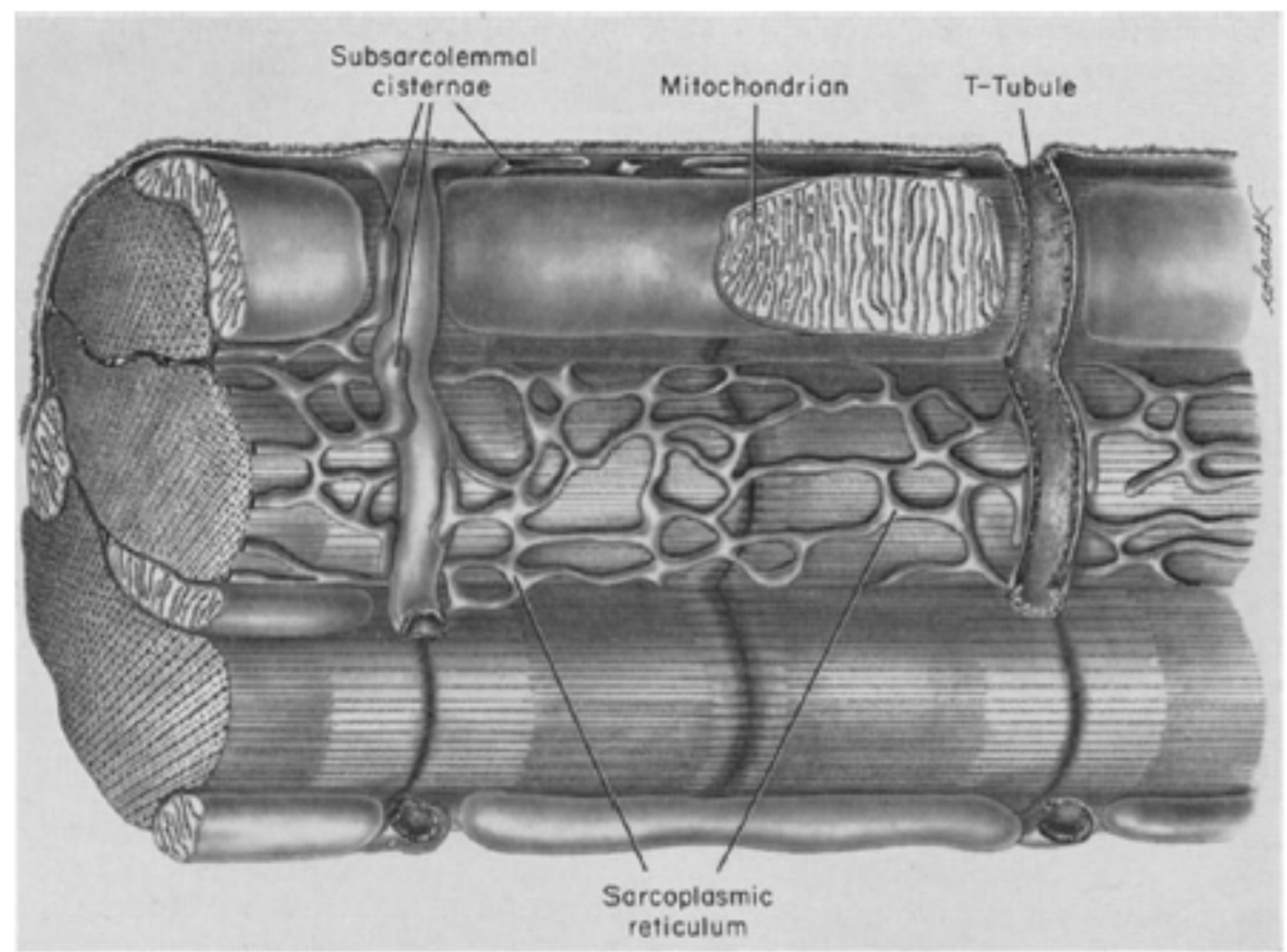

Figure 1.1 Schematic of a Cardiomyocyte

Schematic illustrating key structures involved with ECC within a cardiac muscle cell ${ }^{1}$. 


\subsubsection{The Action Potential}

The resting membrane potential of most cells ranges from $-60 \mathrm{mV}$ to $-95 \mathrm{mV}$, with reference to the extracellular space. For cardiomyocytes, the membrane potential is typically $-85 \mathrm{mV}$ to $-95 \mathrm{mV}$. This membrane potential primarily depends on a gradient of sodium $\left(\mathrm{Na}^{+}\right)$and potassium $\left(\mathrm{K}^{+}\right)$ions driven by a $\mathrm{Na}^{+} / \mathrm{K}^{+}$-ATPase in sarcolemma, as well as the membrane's permeability to $\mathrm{K}^{+}$in the cell's resting state.

ECC begins in the right atrium's sinoatrial (SA) node when pacemaker cells generate an electrical signal. This signal propagates through the chambers of the heart via cardiac muscle cardiac conduction system. This electrical signal triggers a change in membrane potential in contractile cardiomyocytes throughout the heart. This change in membrane potential is known as the action potential (AP).

Action potentials in cardiomyocytes are separated into five distinct phases:

- Phase Zero - Depolarization: The electrical signal generated in the SA node activates voltagegated $\mathrm{Na}^{+}$channels on the sarcolemma. This causes a rush of $\mathrm{Na}^{+}$into the cytoplasm, rapidly depolarizing the cell to membrane potentials of $20 \mathrm{mV}$ to $30 \mathrm{mV}$.

- Phase one - Partial Repolarization: The new membrane potential deactivates the voltagegated $\mathrm{Na}^{+}$channels and activate voltage-gated $\mathrm{K}^{+}$channels. The movement of $\mathrm{K}^{+}$out of the cell causes a small decrease in the membrane potential to values of $20 \mathrm{mV}$ to $-10 \mathrm{mV}$.

- Phase Two - Plateau Phase: $\mathrm{Ca}^{2+}$ enters the cell through voltage gated L-type $\mathrm{Ca}^{2+}$ channels. The $\mathrm{K}^{+}$channels from phase one remain open. The net result is a balance of charge into and 
out of the cell resulting in a long plateau phase around $0 \mathrm{mV}$. The plateau phase is where muscle contraction occurs.

- Phase Three - Repolarization: Closure of L-type $\mathrm{Ca}^{2+}$ channels and the activation of the $\mathrm{Na}^{+} / \mathrm{Ca}^{2+}$ exchanger (NCX), results in $\mathrm{Ca}^{2+}$ leaving the cell. $\mathrm{Ca}^{2+}$-dependent and voltagedependent $\mathrm{K}^{+}$channels open, resulting in a repolarization of the cell.

- Phase Four - Diastolic Phase: Also, known as the resting phase. In this phase, myocytes are at their resting membrane potential. Once the resting potential is reached the voltagedependent $\mathrm{K}^{+}$channels close.

\subsubsection{Contraction of Muscle}

$\mathrm{Ca}^{2+}$ in the cytoplasm, which becomes elevated during the AP, binds to and activates troponin $\mathrm{C}$, a protein linked to actin filaments. Troponin $\mathrm{C}$, upon binding $\mathrm{Ca}^{2+}$, undergoes a conformational change, allowing myosin heads to access the actin filaments. The myosin head hydrolyzes adenosine triphosphate (ATP), shifting its position further down the actin filament.

The shifting of the thin and thick filaments relative to each other has the net effect of shortening the sarcomere. This process, carried out in each sarcomere, results in overall shortening of muscle fibers and contraction of the muscle.

\subsection{Calcium-Induced Calcium Release}

Calcium plays a central role, in both the action potential, as well as in triggering the contraction of muscle. In addition, the amount of $\mathrm{Ca}^{2+}$ in the cell is related to the strength of contraction. Cytosolic $\mathrm{Ca}^{2+}$ is initially increased via L-type $\mathrm{Ca}^{2+}$ channels during the AP, however, this increase in $\mathrm{Ca}^{2+}$ alone is not sufficient to trigger proper 
muscle contraction. The SR within the cell serves as a secondary source of $\mathrm{Ca}^{2+}$, releasing stores of $\mathrm{Ca}^{2+}$ during the AP and triggering muscle contraction.

\subsubsection{Sarcoplasmic Reticulum}

The sarcoplasmic reticulum, a modified endoplasmic reticulum, serves as a $\mathrm{Ca}^{2+}$ storage system within myocytes. The SR forms two distinct regions, one which wraps along the length of the myofibril (longitudinal SR), and the terminal cisternae, which forms the dyadic junction with the T-tubules (junctional SR).

In cardiac muscle, $\mathrm{Ca}^{2+}$ entering the cell during the AP, via the L-type $\mathrm{Ca}^{2+}$ channels, binds to and activates a $\mathrm{Ca}^{2+}$-release channel (CRC) found on the SR. This process is referred to as $\mathrm{Ca}^{2+}$-induced $\mathrm{Ca}^{2+}$ release (CICR).

Junctional SR is near L-type $\mathrm{Ca}^{2+}$ channels found in the T-tubles. In addition, the CRC is more abundant in the junctional SR than in the longitudinal SR. This structural composition allows for near-simultaneous CICR, a critical component to proper ECC. In cardiac muscle, dyadic junctions are the location of all ECC.

Following muscle contraction, $\mathrm{Ca}^{2+}$ levels are returned to diastolic levels. Most $\mathrm{Ca}^{2+}$ $(\sim 70 \%)$ is actively pumped back into the SR through its other major protein, the sarco/endoplasmic reticulum $\mathrm{Ca}^{2+}$-ATPase (SERCA), found primarily (90\%) in the longitudinal SR. The remaining $\mathrm{Ca}^{2+}$ is extruded via the $\mathrm{Na}^{+} / \mathrm{Ca}^{2+}$ exchanger (NCX) and a small portion through plasma membrane $\mathrm{Ca}^{2+}$-ATPases and mitochondrial uniporters. 
CICR is another major distinction between cardiac and skeletal muscle. Skeletal muscle interactions between the L-type $\mathrm{Ca}^{2+}$ channel and the CRC are not as well defined, but are believed to have a physical coupling between them.

\subsection{The SR Ca ${ }^{2+}$-Release Channel: The Ryanodine Receptor}

At the center of $\mathrm{CICR}, \mathrm{ECC}$, and healthy cardiac function is the $\mathrm{SR} \mathrm{Ca}^{2+}$-release channel, the ryanodine receptor. Its name comes from its high affinity for the plant alkaloid, ryanodine, while in its open conformation ${ }^{2}$. Ryanodine receptors are found highly conserved throughout animal species and within the different muscle types.

Different isoforms of the ryanodine receptor are found in the three muscle types: skeletal muscle (RyR1), cardiac muscle (RyR2), and smooth muscle (RyR3). These isoforms of ryanodine receptor share an approximate $70 \%$ sequence homology between them.

The three-dimensional structure was first determined for RyR1 using methods of cryoelectron microscopy in $1995^{3}$. The cytoplasmic assembly of the receptor consists of four identical monomers, together forming a homotetramer ( $290 \AA$ × $290 \AA$ x $130 \AA$ ). There is also a smaller transmembrane assembly $(120 \AA ̊$ X $120 \AA$ x $70 \AA$ ) linking the SR lumen to the myocyte cytoplasm. Altogether, the ryanodine receptor forms the largest known ion channel with a total molecular weight of $2.3 \mathrm{MDa}$. 


\subsubsection{The $\mathrm{Ca}^{2+}$ Spark}

The process of ECC starts with an electrical signal and results in the release of $\mathrm{Ca}^{2+}$ from RyR2 by means of CICR. Many RyR2s are grouped in formations known as couplons at the dyadic junction region. The opening of RyR2 results in a brief flux of $\mathrm{Ca}^{2+}$ out of the $\mathrm{SR}$ into the cardiomyocyte. This $\mathrm{Ca}^{2+}$ release triggers other RyR2s in the couplon to open. This release of $\mathrm{Ca}^{2+}$ from all RyR2's within a couplon is termed as a $\mathrm{Ca}^{2+}$ spark $^{4}$. $\mathrm{Ca}^{2+}$ sparks can occur spontaneously, where a random RyR2 opening triggers a local $\mathrm{Ca}^{2+}$ spark event. Or, in the case of ECC the entry of $\mathrm{Ca}^{2+}$ into the dyadic junction triggers many couplons resulting in simultaneous $\mathrm{Ca}^{2+}$ sparks in the myocyte. The total $\mathrm{Ca}^{2+}$ flux, from all $\mathrm{Ca}^{2+}$ sparks, referred to as the $\mathrm{Ca}^{2+}$ transient, is responsible for muscle contraction $^{5}$.

\subsubsection{Modulation of RyR2}

Release of $\mathrm{Ca}^{2+}$ from the SR during ECC is a highly complex process subject to modulation from cellular processes, associated proteins, cellular conditions, and nonphysiological elements.

In its native state, RyR2 exists as a complex of many associated proteins which together regulate its function:

- Calmodulin: Ubiquitous $\mathrm{Ca}^{2+}$-binding protein that binds to and modifies the state of RyR. Discussed further in section 1.6. 
- FKBP12: 12 kDa FK506 binding protein FKBP12 is mainly associated with RyR1, stabilizing the closed conformation of the channel ${ }^{6}$.

- FKBP12.6: 12.6 kDa FK506 binding protein. FKBP12.6 mainly functions as a regulator of RyR2, stabilizing the closed conformation ${ }^{7}$. FKBP12 is found at much higher concentrations in RyR2 than FKBP12.6, but RyR2's affinity for FKBBP12.6 is approximately 500 times that of FKBP12's, making FKBP12.6 the main regulator of the channel ${ }^{8}$.

- Calsequestrin: $45 \mathrm{kDa}$ low-affinity $\mathrm{Ca}^{2+}$-binding protein found in the SR lumen. Calsequestrin's main function is to increase the SR's $\mathrm{Ca}^{2+}$ storage capacity. Each calsequestrin molecule binds up to fifty $\mathrm{Ca}^{2+}$ ions ${ }^{9}$.

- Triadin and Junctin: $35 \mathrm{kDa}$ protein, triadin and $26 \mathrm{kDa}$ protein, junctin are found in the junctional SR. Both serve as anchors, coupling to calsequestrin and RyR2. Binding affinity for calsequestrin to RyR2 is increased in the presence of low $\mathrm{Ca}^{2+} .10$

- Dihydropyridine Receptor (DHPR): The DHPR (more commonly known by voltage-gated Ltype $\mathrm{Ca}^{2+}$ channel) is a $100 \mathrm{kDa}$ protein involved in the AP as well as in triggering CICR. The association between DHPR and RyR2 is not direct, as is believed to be the case in RyR1.

Cellular processes lead to phosphorylation of RyR2 by certain proteins. This phosphorylation is known to modulate channel activity. Proteins known to play a role in phosphorylation of RyR2 include:

- Protein Kinase A (PKA): a 96 kDa protein which anchors to RyR2 through the muscle A kinase anchoring protein (mAKAP). Elevated cAMP levels in the cell activate PKA. Once activated, PKA phosphorylates RyR2 leading to increased activity ${ }^{11}$. Hyperphosphorylation of RyR2 by PKA is strongly correlated with RyR2-dysfunction. The exact mechanism by which phosphorylation leads to dysfunction is still debated in the literature (discussed in further detail in section 1.5).

- $\mathrm{Ca}^{2+} /$ Calmodulin-Dependent Protein Kinase 2 (CaMKII): CaMKII is a protein kinase which becomes activated upon binding calmodulin in its $\mathrm{Ca}^{2+}$-bound form. Upon activation, CaMKII 
can phosphorylate RyR2. CaMKII phosphorylation of RyR2 has been shown to both increase ${ }^{12}$ and decrease ${ }^{13}$ channel activity.

- Protein Phosphatase 1 and 2a (PP1 and PP2a): PP1 and PP2a regulate channel activity by dephosphorylating the channel, maintaining proper cellular homeostasis ${ }^{14}$.

In addition to proteins that associate with RyR2, there are many other physiological and non-physiological modulators of RyR2. Some of the more important modulators include:

- Ryanodine: High affinity plant alkaloid that is believed to bind in the pore of ryanodine receptors ${ }^{15}$. Open receptors bind ryanodine with a higher affinity and can lock the channel in an open state ${ }^{16}$.

- Caffeine: Activates ryanodine receptor by making the channel mores sensitive to activation by $\mathrm{Ca}^{2+} \cdot{ }^{17}$

- Ruthenium Red: Inhibitor of the channel

- Calcium: $\mathrm{Ca}^{2+}$ is the key regulating ion of RyR2, governing its role in ECC. RyR2 has $\mathrm{Ca}^{2+}$ binding sites on both the cytoplasmic and luminal faces. When $\mathrm{Ca}^{2+}$ levels in the cytosol are low (approximately $1 \mu \mathrm{M}$ ), a high affinity $\mathrm{Ca}^{2+}$ binding site on RyR2 activates the channel. At higher $\mathrm{Ca}^{2+}$ concentrations (> $\left.100 \mu \mathrm{M}\right) \mathrm{Ca}^{2+}$ binds to a lower affinity site which will inhibit the channel ${ }^{18}$.

- Magnesium: $\mathrm{Mg}^{2+}$ inhibits RyR2 by competing with $\mathrm{Ca}^{2+}$ for high affinity binding sites ${ }^{19}$. It is believed to work by direct inhibition when binding to the low-affinity $\mathrm{Ca}^{2+}$ site. At the highaffinity site it is thought to compete with $\mathrm{Ca}^{2+}$, which prevents activation by $\mathrm{Ca}^{2+}$, resulting in a more closed state 20,21 .

- Tetracaine: A local anesthetic which can directly inhibit RyR2 activity22. 


\subsubsection{RyR2-Dysfunction}

Proper ECC is dependent on intricate cardiac structures, various protein interactions, and critical timing of cellular processes. Disrupting any of these things can cause issues in ECC, leading to serious complications including, arrhythmia, heart failure, and death.

There are a vast number of disorders that lead to electrical instability of the heart. The exact mechanisms that result from these disorders in many cases is poorly understood. One underlying factor common to these disorders is the mishandling of $\mathrm{Ca}^{2+}$ within the cell.

Dysfunction of RyR2 can lead to a leak of $\mathrm{Ca}^{2+}$ during diastole. This $\mathrm{Ca}^{2+}$ leak can trigger the $\mathrm{Na}^{+} / \mathrm{Ca}^{2+}$ exchanger, resulting in delayed afterdepolarizations (DADs) which may lead to arrhythmia ${ }^{23}$. In addition, RyR2-Ca ${ }^{2+}$ leak can lead to increased expression of the $\mathrm{Na}^{+} / \mathrm{Ca}^{2+}$ exchanger seen in failing hearts ${ }^{24}$, reduced SERCA activity ${ }^{25}$ and decreased SR$\mathrm{Ca}^{2+}$ stores. Decreases in SR Ca ${ }^{2+}$ reduces the $\mathrm{Ca}^{2+}$ transient, resulting in weaker contractile force associated with heart failure. These changes create a positive feedback system which if left untreated can degenerate into arrhythmia and heart failure.

There are several diseases characterized by improper $\mathrm{Ca}^{2+}$-cycling due to $\mathrm{RyR} 2 \mathrm{Ca}^{2+}$-leak. This work focuses on RyR2-dysfunction associated with catecholaminergic polymorphic ventricular tachycardia (CPVT). 


\subsection{Catecholaminergic Polymorphic Ventricular Tachycardia (CPVT)}

CPVT made its first appearance in the literature in 1978. It is a genetic, arrhythmogenic disorder affecting one in every ten thousand people. Most individuals with the disease will experience first onset of symptoms within the first two decades of life. The first symptoms often include dizziness and syncope, but can also lead to ventricular fibrillation and sudden cardiac death. Without treatment, CPVT has a 30\%-50\% mortality rate by the third decade of life $\mathrm{e}^{26}$.

Electrocardiograms do not typically show signs of CPVT. Stress testing is necessary to see the characteristic bidirectional ventricular tachycardia associated with the disease. The best method of diagnosing CPVT is genetic testing for those individuals whose family have a history of the disease. With no prior knowledge of the disease and the dangers associated with first onset, CPVT is one the leading causes of sudden cardiac death, especially among children.

The different forms of the disease deal with mutations in four different proteins, all of which are associated with RyR2:

- CASQ2 is an autosomal recessive form of the disease involving mutations in calsequestrin CASQ2 accounts for $2 \%$ to $5 \%$ of incidences of $\mathrm{CPVT}^{27}$.

- TRDN is an autosomal recessive for of the disease involving mutations in triadin.

- CALM1 is an autosomal dominant form of the disease involving mutations in calmodulin CALM1 accounts for less than $1 \%$ of all CPVT ${ }^{28}$.

- CPVT1 is an autosomal dominant form of the disease involving mutations in RyR2. CPVT1 is the main form of the disease and conservative estimates show that it accounts for $50 \%$ to 
$55 \%$ of all CPVT ${ }^{29}$. CPVT1 is the main area of interest for this work, all further instances of CPVT refer specifically to CPVT1.

\subsubsection{CPVT1}

There are many known mutations in RyR2 that lead to CPVT, resulting in improper function. However, most cases of CPVT occur as a single point mutation occurring in one of four main regions ${ }^{30}$ :

- Domain I (RyR2 amino acid sequence 77-466). Mutations in Domain I account for approximately $18 \%$ of occurrences of CPVT.

- Domain II (RyR2 amino acid sequence 2246-2534). Mutations in Domain II account for approximately $19 \%$ of occurrences of CPVT.

- Domain III (RyR2 amino acid sequence 3778-4959). Mutations in Domain III account for approximately $22 \%$ of occurrences of CPVT.

- Domain IV (RyR2 amino acid sequence 4497-49590). Domain IV accounts for approximately $22 \%$ of occurrences of CPVT.

These four domains are highly conserved across species as well as the differing isoforms of the ryanodine receptor. It is known that mutations in these four domains in RyR1 correspond to central core disease (CCD) and malignant hyperthermia $(\mathrm{MH})$. It has been shown that specific point mutations known to cause CPVT in RyR2 will cause MH when present in RyR1 ${ }^{31}$. This seems to indicate some mechanistic similarities RyR2/RyR1dysfunction and resulting diseases. 


\subsubsection{CPVT Pathway Leading to RyR2-Dysfunction}

When individuals with CPVT experience ventricular tachycardia (VT) it is usually under times of stress and/or heavy exercise. During these periods the body elevates catecholamine levels which trigger a cascade of events which lead to VT.

Epinephrine is raised in the body as a "fight-or-flight" response when under stress. When this occurs, it binds to and activates the beta1-adrenergic receptor on the sarcolemma. Upon activation, G-proteins, coupled to the receptor on the inner membrane, activate adenylyl cyclase. Adenylyl cyclase, once activated, hydrolyzes ATP into 3',5'-cyclic adenosine monophosphate (cAMP). Elevated cAMP levels in the cell activate protein kinase $A(P K A)$, which then phosphorylates RyR2.

It is believed that this phosphorylation of RyR2 leads to a more open state of the channel and in individuals with CPVT this leads to RyR2 dysfunction ${ }^{32}$.

\subsubsection{Treatment for CPVT}

CPVT has no known cure, treatment is typically targeted to preventing the onset of occurrences with medication. Beta blockers are the most common class of drugs used to treat CPVT. Beta blockers work by binding the beta1-adrenergic receptor and preventing epinephrine from activating the receptor. Beta blockers have been shown to decrease occurrence of CPVT symptoms in the short term, but long term use does not affect the mortality rate from the disease ${ }^{33}$. 
Implantation of cardioverter defibrillators (ICD) are used for individuals whose medication is not effective and/or have recurrent syncope. ICD are invasive and do not prevent arrhythmia and run the risk of firing in the absence of arrhythmia.

Another medication, flecainide, can be prescribed for those whom beta blockers are not effective. Flecainide works as a sodium channel and a RyR2 blocker and has been shown to inhibit arrhythmias from CPVT in both mouse ${ }^{34}$ and clinical trials $\mathrm{s}^{35}$, but can also lead to worsened heart rhythm.

The lack of understanding regarding RyR2-dysfunction resulting from CPVT, the high mortality rate associated with CPVT, and the drawbacks of current treatment options, make the understanding and development of new treatments an important pursuit. Furthermore, understanding of mechanisms involved in CPVT may enhance our understanding of other RyR2-dysfunction related diseases.

\subsection{Hypotheses of RyR2 Dysfunction}

The mechanism responsible for RyR2 $\mathrm{Ca}^{2+}$ leak is still not fully understood and is debated in the literature. Here we briefly discuss prevalent theories regarding RyR2dysfunction and their potential role in CPVT.

\subsubsection{FKBP12.6 Dissociation}

One theory of RyR2-dysfunction is the destabilization of the channel by the removal of FKBP12.6. FKBP12.6 has been shown to stabilize $\mathrm{RyR}^{36}{ }^{36}$, and its removal leads to a more open channel. 
Heart failure is often associated with hyperphosphorylated RyR2. Dissociation of FKBP12.6 has been linked to PKA phosphorylation of RyR2 and its removal has been shown to Increase RyR2 $\mathrm{Ca}^{2+}$-sensitivity ${ }^{31}$. This increase in $\mathrm{Ca}^{2+}$-sensitivity may be the underlying cause of RyR2 $\mathrm{Ca}^{2+}$ leak. Furthermore, reduced FKBP12.6 affinity has been seen in RyR2 with known CPVT mutation, R2475 ${ }^{37,38}$.

Although initial evidence seemed to support the theory of FKBP12.6 dissociation leading to RyR2 malfunction in diseased states such as CPVT, it has become more controversial in recent years. Studies have shown differing results involving FKBP12.6 removal via PKA phosphorylation. One study showed that phosphorylation of RyR2 did not cause FKBP12.6 to dissociate ${ }^{39}$. The R247S mutation has been shown to increase FKBP12.6 affinity ${ }^{40}$ and other studies involving CPVT mutations have shown no reduced affinity for FKBP12.6 $6^{41,42}$.

\subsubsection{Oxidation}

Glutathione/glutathione disulfide (GSH/GSSG) ratios within the cell maintain the redox potential. Redox potential is a measure of how easily something can become oxidized or reduced. $\mathrm{Ca}^{2+}$ handling within cardiomyocytes is known to be influenced by the redox potential of the cell. There are known to be between $300-400$ cysteine residues with thiol side chains on RyR2 which are sensitive to oxidation ${ }^{43}$.

It has been hypothesized that these thiol residues play a critical role in the state of RyR2. A strong correlation has been shown between inhibition of the receptor in the presence 
of strong electron donors and activation of the channel when treated with electron acceptors $^{44}$.

Reactive oxygen and nitrogen species such as $\mathrm{H}_{2} \mathrm{O}_{2}$ and NO influence the GSH/GSSG ratio. These species become elevated due to factors such as exercise, diet, and environmental factors. If the ROS and RNS become too high cells undergo oxidative stress, which can lead to a destabilized channel.

\subsubsection{Domain Unzipping}

Domain unzipping, first proposed by Ikemoto and Yamamoto ${ }^{45}$, refers to an interaction between the N-terminal and central domain of RyR2. These domains remain "zipped" when RyR2 is in a stabilized state. In a malfunctioning receptor, it is believed that these domains become "unzipped" and the channel becomes more sensitive to activators such as $\mathrm{Ca}^{2+}$. It has been shown that a single point mutation in either of these domains can lead to a heightened sensitivity to channel activators. Failing hearts with reduced SR $\mathrm{Ca}^{2+}$ content, and diastolic $\mathrm{Ca}^{2+}$ leak have been accompanied with a weakened domain interaction ${ }^{46}$.

\subsubsection{Calmodulin Dissociation}

Recently, the literature has suggested that dissociation of CaM from RyR2 may be a critical component in increased RyR2 $\mathrm{Ca}^{2+} \mathrm{leak}^{47}$. As the primary focus of this work, CaM and its relation to RyR2-dysfunction in CPVT will be discussed in detail. 


\subsection{Calmodulin}

Calmodulin (CaM) is a highly conserved and ubiquitous $\mathrm{Ca}^{2+}$-binding protein. $\mathrm{CaM}$ is involved in regulating numerous cellular processes. Its structure was first determined by X-ray crystallography in $1985^{48}$ as well as NMR in $1995^{49}$. CaM has 148 residues and a total molecular weight of $16.7 \mathrm{kDa}$.

Its structure consists of two globular EF hands which bind $\mathrm{Ca}^{2+}$ ions, two at each globular head. The two globular heads are connected by a flexible helix tether. When $\mathrm{Ca}^{2+}$ binds to CaM, it undergoes a conformational change, exposing hydrophobic residues. In its $\mathrm{Ca}^{2+}$-bound state, $\mathrm{CaM}$ interacts with over thirty target proteins including: Adenylyl Cyclase, Myosin Light Chain Kinase, Phosphodiesterase, L-Type Ca ${ }^{2+}$ Channels, and the Ryanodine Receptor.

In cardiomyocytes, CaM concentrations have been reported to be between 2-5 $\mu \mathrm{M}^{50}$. Approximately $95 \%$ of CaM is bound to its target proteins in the cell, $20 \%$ is believed to be bound to RyR2 ${ }^{51}$. Free CaM in cardiomyocytes ranges between $50-75 \mathrm{nM}^{52}$. 


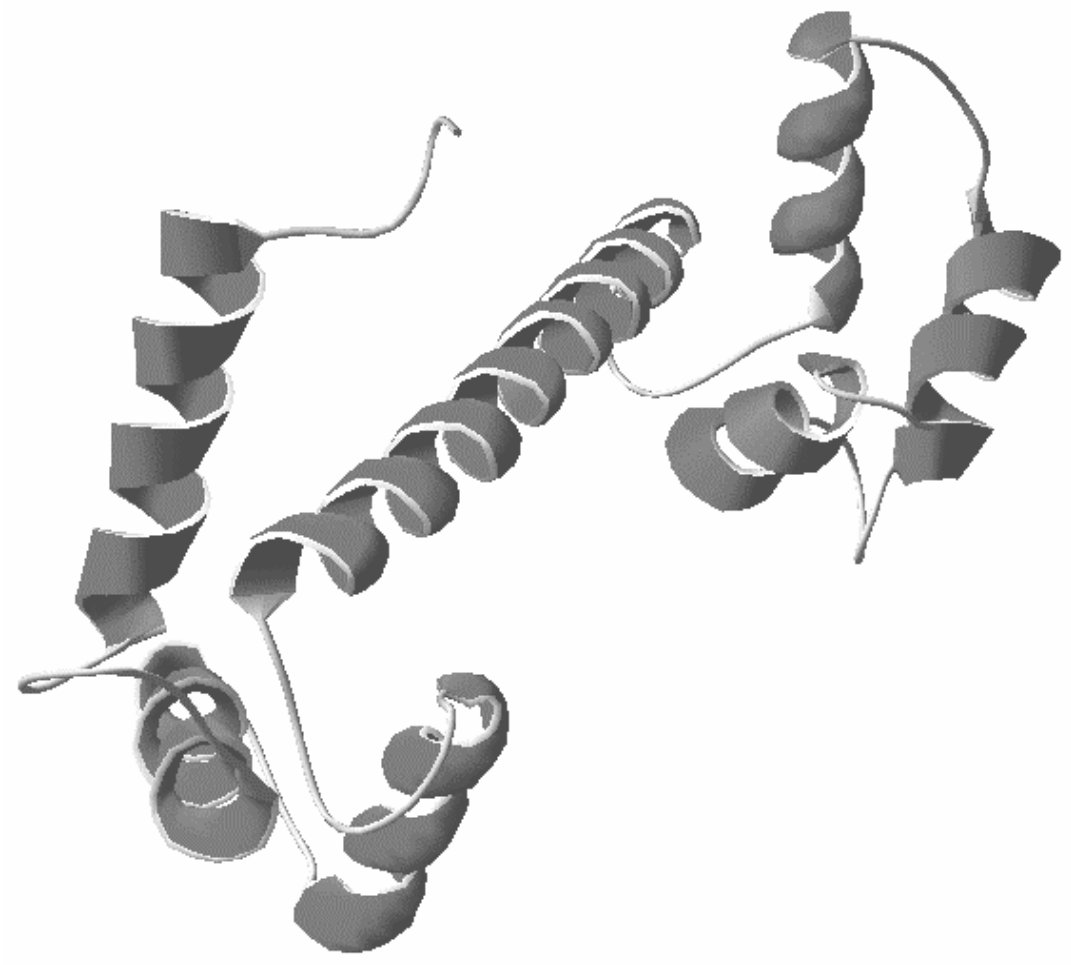

Figure 1.2 Structure of Calmodulin

3-Dimensional structure of CaM in its $\mathrm{Ca}^{2+}$-free form. Structure from PDB source ${ }^{53}$. 


\subsubsection{Calmodulin Interaction with the Ryanodine Receptor}

CaM is known to be a modulator of both skeletal ${ }^{54}$ and cardiac ryanodine receptors ${ }^{55}$. It is also believed to interact with RyR3 ${ }^{56}$ but has been poorly characterized. CaM's effect on both RyR1 and RyR2 will be discussed here to contrast potential correlations between diseases, as well as to highlight discrepancies in studies involving CaM and RyR2.

\subsubsection{Calmodulin Binding to the Ryanodine Receptor}

RyR1 binds CaM in both its $\mathrm{Ca}^{2+}$-free (ApoCaM) and $\mathrm{Ca}^{2+}$-bound (CaCaM) forms ${ }^{57}$. Furthermore, RyR1 has been shown to bind both forms at a single, high affinity, binding site corresponding to RyR1 residues $3614-3643^{58}$. CaM, in either form, binds to RyR1 with a stoichiometric ratio of four CaMs per RyR1 tetramer, or one CaM per monomer ${ }^{59}$. Like RyR1, CaM has been shown to have one, high affinity, binding site on RyR2, corresponding to residues $3583-3603$ in both its ApoCaM and CaCaM form ${ }^{60}$. Most reports have shown that four CaM bind per RyR2 tetramer in purified RyR2. One study showed that in its native form, RyR2 bound 8 CaMs per tetramer ${ }^{61}$.

\subsubsection{Calmodulin Modulation of the Ryanodine Receptor}

ApoCaM is a known activator of RyR $1^{62}$ and CaCaM is an inhibitor of RyR $1^{63}$. As in the case of RyR1, CaCaM is an inhibitor of RyR2 ${ }^{64}$. However, ApoCaM's effect on RyR2 is still poorly understood, with many conflicting results reported in the literature. 
$\mathrm{Ca}^{2+}$ release from cardiac SR vesicles showed no change in the presence $1 \mu \mathrm{M}$ ApoCam, identical experiments carried out on skeletal SR showed that ApoCaM increased rate of $\mathrm{Ca}^{2+}$ release, indicating activation ${ }^{65}$. This study went on to show that $\mathrm{Ca}^{2+}$-dependent activation of RyR1 was shifted to lower $\mathrm{Ca}^{2+}$ levels ( $\mathrm{kd}=360 \mathrm{nM}$ to $\mathrm{kd}=60 \mathrm{nM}$ ), again, no change in $\mathrm{Ca}^{2+}$ activation was seen in RyR2 under identical conditions.

In a different study looking at RyR2 single channel activity, it was seen that in the presence of $2 \mu \mathrm{M}$ cytosolic $\mathrm{Ca}^{2+}, \mathrm{CaM}$ reduced the open probability of the channel by 25-fold, indicating that CaCaM acts as strong inhibitor of RyR2. When the same experiment was performed with a mutant $\mathrm{CaM}, \mathrm{CaM}_{\mathrm{D} 1234}$, incapable of binding $\mathrm{Ca}^{2+}$, no change in RyR2 open probability was seen ${ }^{66}$.

Balshaw, et al. showed that unlike previous reports, CaM inhibited ryanodine binding at low $(<1 \mu \mathrm{M}) \mathrm{Ca}^{2+}$ levels, making the channel less sensitive to $\mathrm{Ca}^{2+}$ activation $^{67}$. Table 1.1 summarizes CaM's effect and interaction with RyR1 and RyR2.

Table 1.1 Summary of Calmodulin's Effect on RyR1 and RyR2

\begin{tabular}{|l|l|l|}
\hline & RyR1 & RyR2 \\
\hline ApoCaM Modulation & Activator & Differing Results \\
\hline CaCaM Modulation & Inhibitor & Inhibitor \\
\hline ApoCaM Stoichiometry & 4 per Tetramer & 4 per Tetramer \\
\hline CaCaM Stoichiometry & 4 per Tetramer & 4 or 8 per Tetramer \\
\hline
\end{tabular}




\subsubsection{Calmodulin and RyR2-Dysfunction}

Calmodulin is known to be involved in the regulation of RyR2 conformation. Growing evidence in the literature suggests that cardiac diseases caused by RyR2-dysfunction, like CPVT, may be due to CaM dissociation from RyR2, resulting in improper $\mathrm{Ca}^{2+} \mathrm{Cycling}$ during ECC.

Early hypotheses in the literature suggested that CaM may play a role in maintaining normal ECC, terminating the release of $\mathrm{Ca}^{2+}$ from SR by inhibiting RyR2 while in its $\mathrm{CaCaM}$ form ${ }^{68}$. Evidence in the literature has since supported this hypothesis.

Studies have shown that RyR2's affinity for CaM is reduced when mutations are made in RyR2's CaM-binding region. These mutations result in sustained $\mathrm{Ca}^{2+}$ transients, reduced CaCaM inhibition at the single channel level, and reduced $\mathrm{CaCaM}$ inhibition in ${ }^{3} \mathrm{H}-$ ryanodine binding ${ }^{69}$. Cardiomyocytes from failing hearts with an increased occurrence of $\mathrm{Ca}^{2+}$ sparks, indicative of RyR2-dysfunction, show that addition of CaM restores the occurrence of $\mathrm{Ca}^{2+}$ sparks to levels seen in healthy cardiomyocytes ${ }^{70}$.

These studies show that improper cardiac function is often accompanied with CaM dissociation from RyR2, and restoring CaM association to RyR2 can restore proper function. This evidence supports the hypothesis that CaM dissociation may be a critical component in RyR2-dysfunction leading to improper $\mathrm{Ca}^{2+}$ cycling in cardiac diseases.

One study used a knock-in (KI) CPVT mouse model (R247S in RyR2) to show that, following PKA phosphorylation, RyR2 had reduced CaM binding affinity. Following 
phosphorylation, the occurrence of $\mathrm{Ca}^{2+}$ sparks increased in the KI mice. In WT mice, phosphorylation of RyR2 had no effect on CaM binding affinity or the occurrence of $\mathrm{Ca}^{2+}$ sparks $^{71}$. Phosphorylation of RyR2 was shown to be equal in both the WT and KI. Interestingly, in the absence of PKA phosphorylation, RyR2 affinity for CaM was equal in both the WT and KI mice. Further studies have shown that binding affinity of CaM to RyR2 is decreased, even in the absence of PKA phosphorylation ${ }^{72,73 .}$

These studies show that RyR2's CaM binding affinity is reduced in CPVT. Following PKA phosphorylation this reduction in affinity is accompanied by arrhythmogenic properties of cells. Normal cells that show no reduction in CaM binding affinity to RyR2 do not result in arrhythmogenic states following PKA phosphorylation.

Faulty interaction between RyR2 and CaM is believed to play an important role domain unzipping, a hypothesis for RyR2-dysfunction and CPVT. The RyR2 sequences believed to be involved domain unzipping overlap strongly with CPVT mutations. The main domain peptide used in domain unzipping studies, DPC10 (RyR2 residues 2460-2495), is known to activate RyR2 leading to an arrythmogenic state. In the presence of DPC10, WT cardiomyocytes have been shown to have reduced CaM binding and increase CaSPF ${ }^{74}$. When the cardiomyocytes were first pre-incubated with CaM and then treated with DPC10, binding of DPC10 was reduced and CASPF did not increase. FRET showed that CaM and DPC10 indicating that the inhibitory effect one has on the other is allosteric. Although, domain unzipping is not the focus of this work, these studies further illustrate 
that CaM plays a role in proper function of RyR2 and that CPVT like states of the channel show faulty CaM interaction. 


\section{Methods and Materials}

\subsection{Preparation of SR Vesicles}

Isolation of sheep cardiac SR vesicles follows a modified protocol from Williams ${ }^{75}$. Sheep hearts collected immediately following anesthetization of sheep are rinsed of excess blood and transported on ice from Oregon Health and Sciences University to Portland State University in a homogenization buffer: $300 \mathrm{mM}$ sucrose, $10 \mathrm{mM}$ PIPES, $0.5 \mathrm{mM}$ PMSF, $1 \mu \mathrm{g} / \mathrm{mL}$ Leupeptin, pH 7.4 with $\mathrm{KOH}$.

Upon arrival to Portland State the atria along with fatty tissue are removed and discarded. The ventricles are cut into $1 \mathrm{~cm}^{3}$ pieces and divided equally into two $500 \mathrm{~mL}$ beakers of homogenization buffer. Each batch is homogenized in a warring blender for 20 seconds at top speed, followed by a second 30 second wait period and blended for a final 20 seconds at top speed.

Following homogenization, both batches are divided equally into 6 Type-19 rotor tubes and spun at $9000 \times$ G in a Beckman type-19 rotor for 20 minutes to remove cell debris. Supernatant is filtered through four layers of cheese cloth and split into 6 type-19 rotor tubes followed by centrifugation at $44,000 \times \mathrm{G}$ for 75 minutes.

The pellet is retrieved and resuspended in a buffer containing: $300 \mathrm{mM}$ Sucrose, 400 $\mathrm{mM} \mathrm{KCl}, 0.5 \mathrm{mM}$ EGTA, $0.5 \mathrm{mM} \mathrm{CaCl}$, $0.5 \mathrm{mM} \mathrm{MgCl}$, $0.5 \mathrm{mM} \mathrm{PMSF}, 1 \mu \mathrm{g} / \mathrm{mL}$ Leupeptin, 25 mM PIPES, pH 7.0 with $\mathrm{KOH}$. Pellet along with buffer are put into a single type-60 centrifuge tube and spun at 50,000 RPM in a type-60 Beckman Rotor for 30 minutes. 
The pellet is resuspended in buffer and homogenized with a glass homgenizer and stored as $300 \mu \mathrm{L}$ aliquots in liquid Nitrogen.

\subsection{Protein Concentration Determination}

Cardiac SR protein concentration is measured using the method of Kalckar ${ }^{76}$. Using an Agilent 8435 UV-Visible spectrophotometer absorbance of set volumes of SR is measured at $230 \mathrm{~nm}, 260 \mathrm{nM}$, and $280 \mathrm{nM}$. These absorbance peaks correspond to the protein backbone, nucleic acid correction, and tyrosine/tryptophan maximum absorbance. Protein concentration is then determined using the following equations:

\section{Equation 2.1}

$$
\begin{aligned}
& S R\left[\frac{m g}{m L}\right]=1.45\left(A b s_{280}\right)-0.74\left(A b s_{260}\right) \\
& S R\left[\frac{m g}{m L}\right]=0.185\left(A b s_{230}\right)-0.075\left(A b s_{260}\right)
\end{aligned}
$$

Equation 2.2

\subsection{Purification of RyR2}

RyR2 was purified and reconstituted into proteoliposomes following a modified protocol of Meissner ${ }^{77}$, for the purification of RyR1. Crude cardiac SR is first separated into junctional (heavy) and longitudinal (light) SR by centrifugation in a SW-28 rotor at $103,000 \times \mathrm{G}$ for 2 hours on a discontinuous sucrose gradient (0.3 M, 0.8 M, 1.0 M, 1.2 M, $1.5 \mathrm{M}, 230 \mu \mathrm{M}$ PMSF, $1 \mu \mathrm{M}$ Leupeptin, 10 mM Imidazole, pH 7.4 with $\mathrm{KOH})$. Heavy SR is collected at the 1.2 M/1.5 $\mathrm{M}$ interface and resuspended 1:1 in resuspension buffer (0.3 M sucrose, $10 \mathrm{mM}$ imidazole, $\mathrm{pH}$ 7.4). Buffer and heavy SR is centrifuged for 
40 minutes at $95,000 \times$ G in a Ti-60 rotor. Supernatant is discarded and pellet is resuspended into the resuspension buffer (less than $5 \mathrm{~mL}$ total). Protein concentration is determined as described in section 2.2. Heavy SR is separated into $200 \mu \mathrm{L}$ aliquots and stored in liquid nitrogen until use.

$12 \mathrm{mg}$ of heavy SR is solubilized to a concentration of $1 \mathrm{mg} / \mathrm{mL}$ in solubilization buffer (CHAPS, PC, NaCl, PIPES, EGTA, Pefabloc, DTT) for 1 hour on ice by stirring. Following solubilzation, $2 \mathrm{mg}$ is placed onto a discontinuous sucrose gradient (7\%-15\% sucrose, $\mathrm{NaCl}$, PIPES, CHAPS, PC, Pefabloc, Leupeptin, DTT, $\mathrm{CaCl}_{2}$, EGTA, CAMP, pH 7.4, prepared in cold room). 6 tubes in total are placed into an SW-28 rotor and centrifuged at $89,454 X$ for 17 hours.

1 of the 6 tubes contains heavy SR incubated with ${ }^{3} \mathrm{H}$-ryanodine. Following centrifugation, $2 \mathrm{~mL}$ fractions are taken from the gradient containing ${ }^{3} \mathrm{H}$-ryanodine, filtered and activity is measured. The fraction containing the highest counts is kept from the other 5 gradients and pooled. The samples are dialyzed at $100 \mathrm{X}$ volume over 44 hours with 3 buffer changes ( $\mathrm{NaCl}$, PIPES, EGTA, $\mathrm{CaCl}_{2}, \mathrm{PMSF}, \mathrm{DTT}$ ) in the cold room. Dialyzed samples are collected and centrifuged in buffer (PIPES, EGTA, $\mathrm{CaCl}_{2}, \mathrm{PMSF}$ ) in a Ti-60 rotor at 163,520XG for 2 hours. Pellet containing purified RyR2 is collected and homogenized in buffer ( $\mathrm{NaCl}$, PIPES, Sucrose), aliquoted into $50 \mu \mathrm{L}$ samples and stored in liquid nitrogen until use in experiments. 


\subsection{Single Channel Recordings}

\subsubsection{Planar Lipid Bilayers}

Planar lipid bilayers were formed using the method originally published by Mueller et. $\mathrm{al}^{78}$. Two chambers representing the myocyte cytoplasm (CIS) and SR lumen (TRANS) are separated by a small aperture. A pipette tip dipped in a lipid mixture is used to create a monolayer over this aperture. After the chambers are filled with solution, a second pipette tip is used to apply a second monolayer over the aperture.

The two monolayers hydrophobic tails will face the hydrophobic solvent separating them, the hydrophilic head groups face the aqueous solution in the chambers. The Van der Walls force between these two monolayers force these two monolayers together pushing out the solvent and forming a bilayer. This spontaneous process of thinning results in bilayers of varying resistances. Capacitance and current leak of the bilayer is monitored. Bilayers may be thinned further by manually dragging a small bubble over the aperture where the bilayer is formed until the desired capacitance is reached. A desired capacitance for a $150 \mu \mathrm{M}$ aperture is between $40 \mathrm{pF}$ to $100 \mathrm{pF}$ and shows a good square wave pattern.

\subsubsection{Solutions and Material}

Bilayer chambers were made from acetyl or delrin in the Portland State University student machine shop. The construction consists of a base which has two openings, one which holds the CIS solutions and one which holds a cylindrical cup which holds the 
TRANS solution. The cup interface between the two chambers is thinned to approximately $150 \mu \mathrm{M}$ and a small hole $(100 \mu \mathrm{M}-200 \mu \mathrm{M})$ is drilled through this interface. It is believed that an aperture roughly the same diameter as the thickness promotes fusion into a bilayer.

The lipid mixture used to create the bilayer is composed of a 5:3:2 mixture of DOPE:DOPS:DOPC (DOPE: 1,2-Dioleoyl-sn-Glycero-3-Phosphoethanolamine; DOPS: 1,2Dioleoyl-sn-Glycero-3[phosphor-L-serine]; DOPC: 1,2-Dioleoyl-sn-Glycero-3phosphocholine) suspended in a hydrophobic solvent (n-decane) at a concentration of 33 mg/ml. Lipids are purchased from Avanti Polar Lipids (Alabaster, USA).

All bilayer work is done with the CIS chamber is filled with $250 \mathrm{mM} \mathrm{KCl}, 15 \mathrm{mM}$ HEPES, and $100 \mu \mathrm{M} \mathrm{CaCl}_{2}$ at $\mathrm{pH}$ 7.4. The TRANS chamber is filled with $25 \mathrm{mM} \mathrm{KCl}$, and $15 \mathrm{mM}$ HEPES, at $\mathrm{pH}$ 7.4. Following vesicle fusion the TRANS chamber is brought to $250 \mathrm{mM} \mathrm{KCl}$ with a $2.5 \mathrm{M} \mathrm{KCl}, 15 \mathrm{mM}$ HEPES, pH 7.4 solution. The CIS chamber's free $\mathrm{Ca}^{2+}$ is chelated to $25 \mu \mathrm{M}$ with EGTA, as determined by the program Bound and Determined. In certain experiments a salt concentration of $400 \mathrm{mM} \mathrm{KCl}$ was used following the same methods as above.

\subsubsection{SR Fusion}

Once a desired bilayer is formed, crude cardiac SR vesicles or purified RyR2 reconstituted into proteoliposomes from sheep heart are added to the CIS chamber, over time these will incorporate into the bilayer resulting in a "fusion event." The initial 
salt gradient is set up to swell the bilayer; this creates a larger surface area promoting protein fusion. It is also believed that the vesicles aggregate at the bilayer surface, the ionic difference on either side of the bilayer will cause $\mathrm{H}_{2} \mathrm{O}$ to pass into the vesicles from the TRANS chamber causing the vesicles to swell and rupture. When the vesicles rupture, they integrate into the bilayer resulting in protein fusion.

After fusion, the chambers are brought to equal ionic concentrations, this prevents further fusions from occurring. It is desirable to have only one channel present at a time due to the increasing instability of the bilayer with more fusions, as well as for analysis purposes. Some data was recorded in the presence of two and in rare cases three active channels.

\subsubsection{Recordings}

After fusion occurs and chambers are equalized, data is ready to be collected. Current is measured via two $\mathrm{Ag} / \mathrm{AgCl}$ electrodes submerged in electrically conducting salt bridges (Agarose, 4M CsCl). The electrodes connect to a pre-amplifier which then connects to a Warner BC535 amplifier. The signal is then digitized through a Digidata 1322A digitizer and the measurements are recorded in ClampEx patch clamp chart recorder (pClamp 9.0). The preamplifier and chambers are housed within a faraday cage which is closed off during data collection. The signal is digitally filtered at $1 \mathrm{kHz}$ low pass filter and sampled at (sampling rate) Recordings are made for no less than 2-3 minutes for each condition, prior to each there is a 30 second period of stirring the solutions in both the CIS and TRANS chambers. 
Recordings are made in the presence of a holding potential of $+36 \mathrm{mV}$ with the TRANS chamber held at virtual ground. RyR2 is not a voltage gated channel but a voltage is applied as a driving force due to the equal ionic concentrations between the two chambers. Certain experiments were performed at different holding potentials to see if there was a voltage dependent effect in the data.

RyR2 is a $\mathrm{Ca}^{2+}$ ion channel, however, $\mathrm{K}^{+}$is used as the charge carrier in bilayer experiments for two reasons. First, the conductance of RyR2 is larger for $\mathrm{K}^{+}$than it is for $\mathrm{Ca}^{2+} \cdot{ }^{79}$ Secondly, $\mathrm{Ca}^{2+}$ modulates the activity of the channel.

Unlike SR vesicles, it is possible for the purified RyR2 reconstituted into proteoliposomes to fuse with the cytoplasmic assembly facing the TRANS chamber. To ensure that all data used is with the cytoplasmic assembly facing the CIS chamber, purified RyR2 is tested for its orientation in the bilayer by monitoring its gating as a function of $\mathrm{Ca}^{2+}$. The cytoplasmic assembly is more sensitive to $\mathrm{Ca}^{2+}$ and activity will decrease as a function of decreasing $\mathrm{Ca}^{2+}$. Channels that were not sensitive to cytoplasmic $\mathrm{Ca}^{2+}$ were not used in the final analysis of the given compound's dose-response.

Artificial lipid membranes are often subject to current leak over time which can lead to bilayer breakage. This leads some data sets to be absent of full dose-response recordings. 


\subsubsection{Analysis}

\subsubsection{Event Detection}

Clampfit software (pClamp 9.0) is used to generate an events list of data using a halfamplitude threshold technique. A baseline level is manually set in the program along the closed state of the recording. A second level is set representing the open state of the channel; in cases where there is more than one channel, more levels can be set to represent openings for multiple channels. Any event that is at least $50 \%$ the open state conductance, is treated as an open event and the program measures the length (dwell time) of the event.

\subsubsection{Open Probability}

The main method of determining a compound's effectiveness in inhibiting RyR2 is determining the open probability (Po) over a range of concentrations. Po represents the probability of a channel being in an open state. A Po of 1 represents a channel is open $100 \%$ of the time and a Po of 0 represents a channel that is completely closed. Clampfit determines the Po as the fractional amount of time the channel is open:

Equation 2.3 $P o=\frac{T o}{T o+T c}$

Equation 2.4 To= $t_{o}^{1}+t_{o}^{2}+t_{o}^{3}+\ldots+t_{o}^{n}$

Equation 2.5 $T c=t_{c}^{1}+t_{c}^{2}+t_{c}^{3}+\ldots+t_{c}^{n}$

Dose-Response curves are generated from the open probabilities and fit to a fourparameter logistic equation. 


\subsubsection{Open and Closed Dwell Times}

In addition to measuring the open probabilities of channels, the open and closed dwell times are examined. Po depends on the open dwell time (To), closed dwell time (Tc), and frequency of fluctuation. The Po can be changed by modifying any of these parameters and to better understand the kinetics of gating and how a compound is changing the Po the average open and closed dwell times are examined.

Using the methods of Sigworth and Sine ${ }^{80}$ open and closed dwell times are compiled into a histogram. Events vs. dwell times are binned logarithmically which centers at the average dwell time.

\subsubsection{Determination of Statistical Significance of Dwell Times}

Statistical significance was determined for the dwell times. Dwell times at each concentrations were compared to the control using a t-test.

Equation 2.6

$$
t=\frac{X_{1}-X_{2}}{S_{X 1 X 2}\left(\sqrt{\frac{1}{n 1}+\frac{1}{n 2}}\right)}
$$

Equation 2.7 $S_{X 1 X 2}=\sqrt{\frac{(n 1-1) S_{X 1}^{2}+(n 2-1) S_{X 2}^{2}}{n 1+n 2-2}}$

Where $n 1, n 1$ are equal to the sample sizes. $X_{1}$ and $X_{2}$ are the averages. $S_{1}$ and $S_{2}$ are the standard deviations. $p$-values were then calculated and a p-value less than 0.05 was taken to be significant. 


\subsection{Ryanodine Binding}

\subsubsection{Equilibrium Binding}

Ryanodine is a plant alkaloid that binds to the ryanodine receptor with high affinity when the receptor is in an open conformation. Tritium labelled Ryanodine $\left({ }^{3} \mathrm{H}-\right.$ Ryandoine) can then be used to probe the "openness" of a population of SR vesicles under varying conditions. Strong inhibitors of the ryanodine receptor should place the receptor in a more closed conformation and therefore there should be less total ryanodine binding.

Varying concentrations of 84F2 and metabolites were combined in test tubes containing binding buffer ( $250 \mathrm{mM} \mathrm{KCl}, 15 \mathrm{mM} \mathrm{NaCl}, 20$ mM PIPES, pH 7.4 with $\mathrm{KOH}$ ), crude cardiac SR vesicles $(0.25 \mathrm{mg} / \mathrm{mL}), 25 \mu \mathrm{M} \mathrm{CaCl}_{2}, 13 \mathrm{nM}$ unlabeled ryanodine, and $2 \mathrm{nM}{ }^{3} \mathrm{H}$ Ryanodine. Test tubes are added to a hot water bath $\left(37^{\circ} \mathrm{C}\right)$ for 3 hours which allows ryanodine binding to reach equilibrium.

The incubation is quenched by rapid filtration through Whatman glass fiber filters using a 24 or 48 cell Brandel Cell Harvester. Filters are washed of excess non-bound ryanodine using a wash buffer $\left(250 \mathrm{mM} \mathrm{KCl}, 20 \mathrm{mM}\right.$ TRIS, $15 \mathrm{mM} \mathrm{NaCl}, 100 \mu \mathrm{M} \mathrm{CaCl}_{2}, \mathrm{pH} 7.4$ with 
$\mathrm{KOH})$. Filters are added to $5 \mathrm{~mL}$ scintillation vials and $3 \mathrm{~mL}$ of CytoScint scintillation fluid is added.

After the vials are shaken for 24 hours, a Beckman LS 6000 was used to measure the activity present in each vial. Using the activity measured, the known total activity used in each sample, and subtracting off for non-specific ryanodine binding (measured with $200 \mathrm{nM}$ unlabeled ryanodine and 4 mM EGTA), concentration of ryanodine bound per milligram of protein is calculated.

\subsubsection{Rate of Binding}

Ryanodine binding rates can be measured similar to equilibrium binding with the following changes: Incubation is started with the addition of ${ }^{3} \mathrm{H}$-ryanodine into the test tubes at $37^{\circ} \mathrm{C}$. Instead of a 3-hour incubation, samples are incubated for different time lengths (typically 5, 10, 15 and 20 minutes) and quenched immediately. Samples are measured as in equilibrium binding except no non-specific binding is subtracted. Each condition is plotted as the amount of ${ }^{3} \mathrm{H}$-ryanodine bound vs. time. Points are fit to a standard linear fit where "a" represents the rate of binding and yo is equivalent to nonspecific binding:

\section{Equation 2.8

$$
y=a x+y_{0}
$$

Rate of binding is examined as a function of concentration of compound to determine if inhibition of binding rate occurs. 


\section{Basis of Study}

$\mathrm{Ca}^{2+}$ leak from SR is often the underlying cause in many cardiovascular disorders. Dysfunction in RyR2, the source of $\mathrm{Ca}^{2+}$ leak, can lead to DADs, arrhythmia, reduced SR$\mathrm{Ca}^{2+}$ load, cardiac restructuring, reduced contractility, heart failure, and death.

CPVT is one disorder where a mutation in RyR2 is characterized by improper $\mathrm{Ca}^{2+}$ handling. Treatment for CPVT, as well as other RyR2-associated cardiovascular diseases, has primarily relied on medication targeted at preventing the onset of hyperadrenergic states of cardiomyocytes.

RyR2-dysfunction, as the main source of improper $\mathrm{Ca}^{2+}$ cycling in many of these disorders makes it a prime target for treatment. Preventing the leak of $\mathrm{Ca}^{2+}$ from RyR2, with drugs specifically designed to inhibit the channel, could be a novel approach of treatment for not only CPVT, but other RyR2/RyR1-associated diseases. In addition, by understanding the mechanisms and effects of these drugs, our understanding of RyR2dysfunction can be enhanced.

\subsection{Design of Therapeutic Drugs}

Treatment of RyR2 $\mathrm{Ca}^{2+}$-leak relies upon drugs which strongly inhibit the channel. There are many known inhibitors and activators of RyR2. Among these compounds a trend was observed. Compounds that inhibited RyR2 tended to be electron donating, while activators of RyR2 were electron acceptors. It was further shown that a correlation 
existed between the strength of the donor/acceptor and the potency of inhibition/activation ${ }^{81}$.

Working with the hypothesis that strong electron donors tend to be potent inhibitors of RyR2, many compounds were designed to target and inhibit RyR2. Using the structure of tetracaine as a base, a known inhibitor of RyR2, derivatives designed to have stronger electron donating properties, were systematically tested in a $\mathrm{Ca}^{2+}$-spark frequency (CaSPF) screening assay. Those compounds which exhibited promising results were further screened in a variety of assays for their potential to treat RyR2-dysfunction.

\subsubsection{F2 and Metabolites}

The primary focus of this work is on the novel compound, 84F2. 84F2 was designed with an ester linkage to allow easy passage across the sarcolemma. This ester linkage can be hydrolyzed in vivo, which upon cleavage breaks into the compounds, $78 \mathrm{~F} 3$, and 2 (Diethylamino)ethanol (DEAE). 


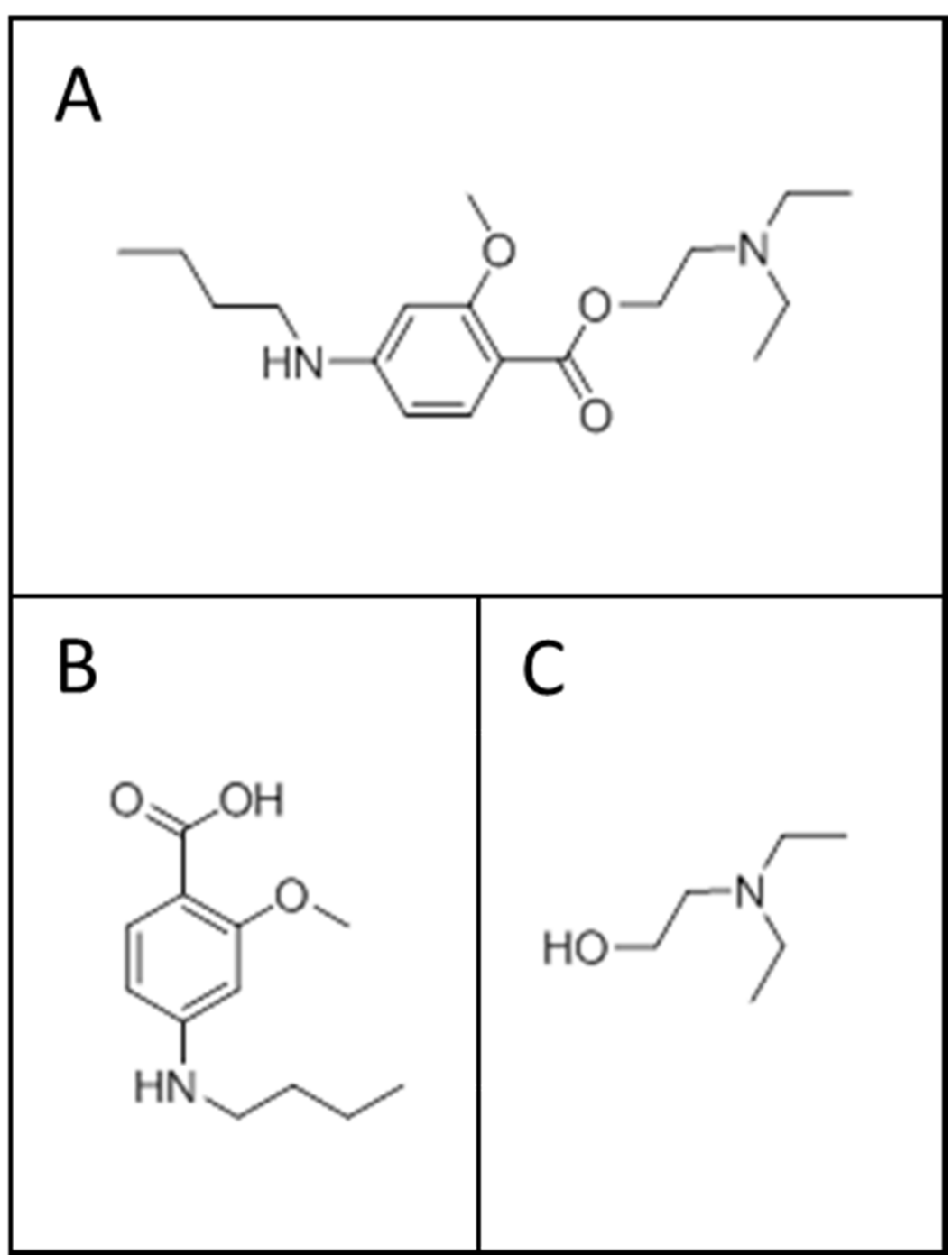

Figure 3.1 Structures of 84F2, 78F3, and DEAE

Chemical structures of 84F2 and its two metabolites following the ester cleavage. A) 84F2. B) 78F3. C) DEAE 


\subsection{Initial Results}

Experiments involving 84F2 were first carried out by our collaborators in the Wehren's Lab at Baylor School of Medicine. These laid the foundation for the work that follows.

\subsection{1 $\mathrm{Ca}^{2+}$ Spark Assay}

When the SR releases $\mathrm{Ca}^{2+}$, via RyR2, local spikes in $\mathrm{Ca}^{2+}$ trigger other nearby RyR2 molecules to open and release their $\mathrm{Ca}^{2+}$, resulting in a $\mathrm{Ca}^{2+}$ spark. Using a $\mathrm{Ca}^{2+}{ }^{2+}$ sensitive fluorescent dye, this release of $\mathrm{Ca}^{2+}$ can be measured. The frequency of the $\mathrm{Ca}^{2+}$ sparks gives insight into how the cell is functioning. An increase in the $\mathrm{Ca}^{2+}$ spark frequency (CaSPF) is characteristic of an arrhythmogenic state of the cell.

To initiate the increase in $\mathrm{Ca}^{2+}$ spark frequency (CaSPF) cells are treated with isoproterenol, mimicking a hyperadrenergic state associated with an arrhythmia. Healthy cells have very few $\mathrm{Ca}^{2+}$ sparks, even under adrenergic stimulation. Cells with RyR2-dysfunction, will show an increase in CaSPF following treatment with isoproterenol.

Cardiomyocytes taken from mice with CPVT mutation, RyR2 ${ }^{\mathrm{R} 176 \mathrm{O} /+}$, which have been shown to have increased CaSPF under adrenergic stimulation ${ }^{82}$, were used to test compounds for their effectiveness in treating arrhythmias resulting from CPVT.

84F2 was tested in a dose-dependent manner using these RyR2 ${ }^{\mathrm{R} 176 \mathrm{O} /+}$ cells. Figure 3.2 shows the dose-response of $84 \mathrm{~F} 2$ tested on CaSPF from RyR2 ${ }^{1760 /+}$ cells. $84 \mathrm{~F} 2$ shows a strong potency in inhibition of the CaSPF, resulting in an $\mathrm{IC}_{50}=35.40 \mathrm{nM}$. 
$84 \mathrm{~F} 2$ resulted in no decrease of Ca spark amplitude that was induced by field stimulation (data not shown here). The amplitude reflects changes in the $\mathrm{Ca}^{2+}$ transients related to ECC. This result with $84 \mathrm{~F} 2$ indicates that normal ECC was not altered. In addition to $84 \mathrm{~F} 2$, its metabolite, $78 \mathrm{~F} 3$, was tested in a manner identical to 84F2. Figure 3.3 shows the dose-response of $78 \mathrm{~F} 3$ on $\mathrm{RyR} 2^{\mathrm{R} 176 \mathrm{O} /+}$ cells in an arrhythmogenic state. $78 \mathrm{~F} 3$ shows an inhibition of the CaSPF with an $\mathrm{IC}_{50}=200 \mathrm{nM}$. 


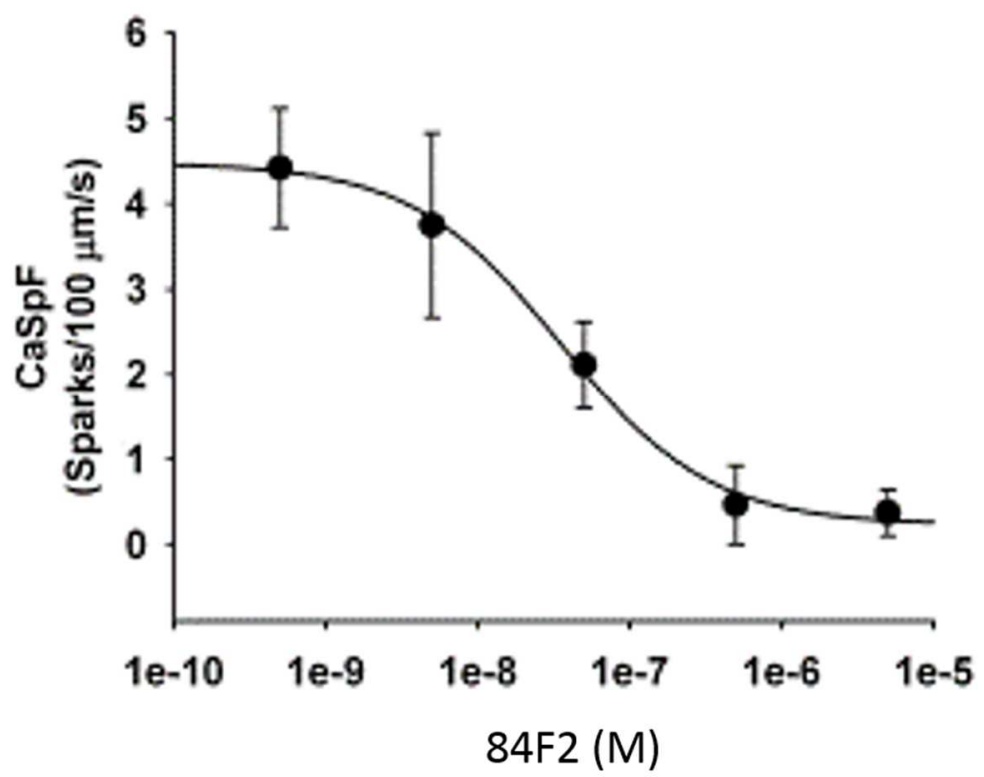

Figure 3.2 84F2 Inhibits CaSPF from RyR2 ${ }^{\mathrm{R} 176 \mathrm{Q} /+}$ Cardiomyocytes

84F2 inhibits stimulated $\mathrm{Ca}^{2+}$ spark activity in $\mathrm{RyR2}^{\mathrm{R} 176 \mathrm{O} /+}$ cardiomyocytes from mice. Cardiomyocytes with increased CaSPF, induced by adrenergic stimulation, were potently inhibited by $84 F 2$. IC $C_{50}=35.40 \mathrm{nM}$.

This work was carried out in the Wehren's Lab at Baylor School of Medicine. 


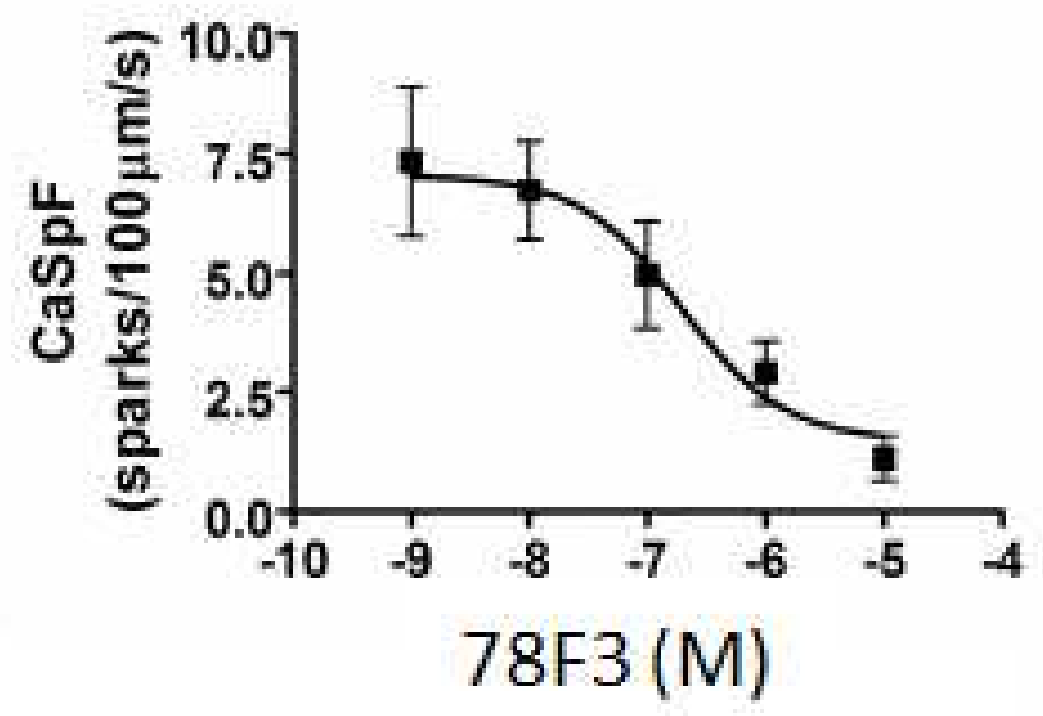

Figure 3.3 78F3 Inhibits CaSPF from RyR2R176Q/+ Cardiomyocytes

$78 \mathrm{~F} 3$ inhibits stimulated $\mathrm{Ca}^{2+}$ spark activity in $\mathrm{RyR}^{\mathrm{R} 1760 /+}$ cardiomyocytes from mice. Cardiomyocytes with increased CaSPF, induced by adrenergic stimulation, were potently inhibited by $78 \mathrm{~F} 3 . \mathrm{IC}_{50}=200 \mathrm{nM}$. This work was carried out in the Wehren's Lab at Baylor School of Medicine. 


\subsubsection{Monitoring Arrhythmia at Whole Animal Level}

$84 \mathrm{~F} 2$ was also tested at the whole animal level. Simultaneous recordings of 6 -lead ECG as well as intercardiac electrograms are simultaneously recorded in adult (2-3month old) mice with the RyR2 $2^{\mathrm{R} 1760 /+}$ mutation. After induction of anesthesia (1.5\% isoflurance in $95 \% \mathrm{O}_{2}$ ), baseline and intercardiac electrogram recordings are made. Next, inducibility of ventricular tachycardia is determined using overdrive pacing and premature stimulus. These measurements are carried out both with and without intraperitoneal (i.p.) injection of 84F2.

Figure 3.4 shows $84 \mathrm{~F} 2$ 's effect at suppressing arrhythmia at the whole animal level. Figure 3.4A shows that wild type (WT) mice exhibit normal sinus rhythm, while sinus rhythm from RyR2 ${ }^{\mathrm{R} 1760 /+}$ mice exhibit bidirectional and sustained ventricular tachycardia (VT) following treatment with isoproterenol and electrical stimulus (indicated by arrows). Following I.P. injection of 84F2, VT was clearly diminished in RyR2 ${ }^{\mathrm{R} 1760 /+}$ mice. Figure 3.4B summarizes the results, showing that the incidence of induced VT is greatly reduced in RyR2 ${ }^{\mathrm{R} 176 \mathrm{O} /+}$ mice, following treatment with $2.5 \mu \mathrm{g} / \mathrm{kg} 84 \mathrm{~F} 2$. 
A

L2-ECG

Atrial electrogram

Ventricular electrogram
WT

(Sinus rhythm)

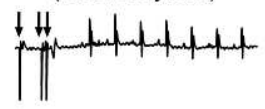

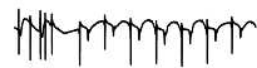

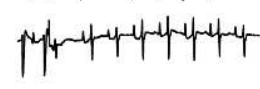

RyR2 $2^{\mathrm{R} 176 \mathrm{Q} /+}+$ placebo

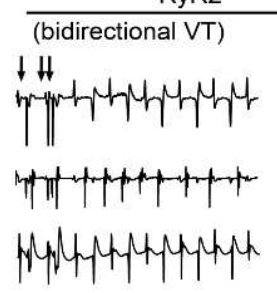

(sustained VT)

$\downarrow \downarrow$

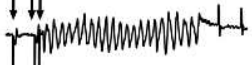

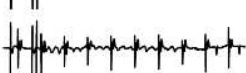

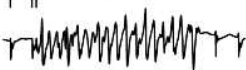

$\mathrm{RyR}^{\mathrm{R} 176 \mathrm{Q} /+}+02-84 \mathrm{~F} 2$

$\downarrow$ H
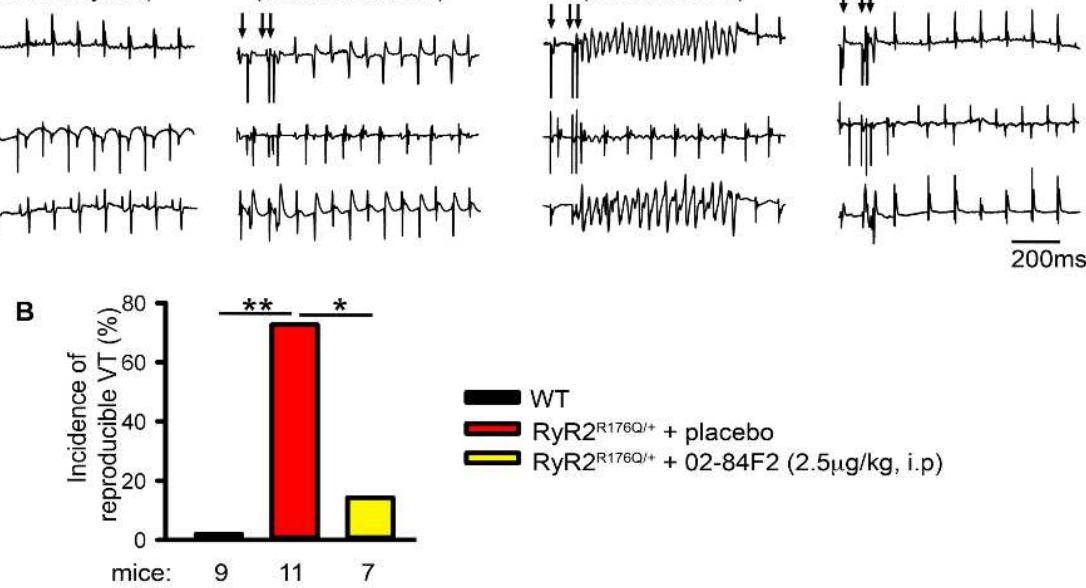

Figure 3.4 84F2 Suppresses the Induction of Ventricular Tachycardia (VT) in RyR2 $^{\mathrm{R} 176 \mathrm{Q} /+}$ mice

A) Representative simultaneous recordings of surface ECG and intracardiac electrograms after S1-S2 extrastimuli (indicated by arrows) revealed bidirectional and sustained VT in RyR2 ${ }^{\mathrm{R} 1760 /+}$ mice treated with placebo, or sinus rhythm in RyR2 ${ }^{\mathrm{R} 1760 /+}$ mice treated with 02-84F2. B) Bar graph summarizing incidence of reproducible VT induced by S1-S2 extrastimuli and significant decrease in VT treated with 02-84F2. This work was carried out in the Wehren's Lab at Baylor School of Medicine. 


\subsection{Goal of Study}

Initial experiments from our collaborators show that 84F2 potently inhibits arrhythmogenic states arising from adrenergic stimulation of CPVT cells, as well as significantly diminish the incidence of VT at the whole animal level. In addition, cells and whole animals treated with 84F2 show normal ECC.

These results show important characteristics of what an ideal drug designed to treat arrhythmia should have: alleviation of arrhythmia and no detrimental effects on cardiac function following treatment. $84 \mathrm{~F} 2$, having these properties, makes it a promising candidate for therapeutic treatment for CPVT and other RyR2-mediated $\mathrm{Ca}^{2+}$ leak diseases.

The results also present a fundamental question underlying RyR2-dysfunction. RyR2 leak of $\mathrm{Ca}^{2+}$ can lead to cardiac disorders, however, the release of $\mathrm{Ca}^{2+}$ from RyR2 is essential to ECC. By studying the mechanisms involved in 84F2's inhibition of RyR2, our understanding of what leads to RyR2-dysfunction may be enhanced. 


\section{Results: Determination if 84F2 Directly Inhibits Purified RyR2}

The decrease in CaSPF from RyR $2^{\mathrm{R} 1760 /+}$ cardiomyocytes indicates that RyR2 is inhibited in the presence of nanomolar concentrations of 84F2. However, it is not clear whether this inhibition of RyR2 is due to an indirect or direct effect on RyR2 activity. 84F2 was designed around the known RyR2 inhibitor, tetracaine, suggesting that the results seen at the cellular and whole animal level are the result of the direct inhibition of RyR2 by 84F2.

Hypothesis 1: 84F2's mechanism of treating arrhythmia is through the direct inhibition of RyR2.

To test this hypothesis, purified RyR2 single channels were used in a planar lipid bilayer to determine if 84F2 directly inhibits RyR2. 


\subsection{F2 Inhibition of Purified RyR2 at the Single Channel Level}

RyR2, purified and reconstituted into proteoliposomes as described in the methods, was incorporated into a planar bilayer and single channel activity was measured as a function of $84 \mathrm{~F} 2$ concentration.

Figure 4.1 shows representative single channel recordings of an experiment carried out with 84F2. Trace A shows a control recording done in the presence of $25 \mu \mathrm{M}$ cytosolic $\mathrm{Ca}^{2+}$ and no 84F2. This recording yielded an average open probability (Po) of 0.97, indicating that the channel spends $97 \%$ of the time in an open state. Subsequent recordings $B, C$, and $D$ show channels in the presence of $3 n M, 5 n M$, and $35 n M 84 F 2$. These recordings yield channels Po measurements of $0.43,0.28$, and 0.05 , respectively. Subsequent recordings on this channel at higher 84F2 concentrations completely inhibited all channel activity. 84F2 is seen here to inhibit purified RyR2 in a concentration-dependent manner.

Further channels were examined over a broad range of $84 \mathrm{~F} 2$ concentrations and a doseresponse curve was generated. The half-maximal inhibition concentration, or $\mathrm{IC}_{50}$ was calculated (as described in the methods) and used as a quantitative measure of 84F2's potency.

Figure 4.2 shows the dose-response curve for $84 \mathrm{~F} 2$ inhibition of purified RyR2 at the single channel level. 84F2 was added at concentrations ranging from $1 \mathrm{nM}$ to $100 \mu \mathrm{M}$ 
for a total 12 channels. The pIC 50 of $84 \mathrm{~F} 2$ is $8.09 \pm 0.139\left(\mathrm{IC}_{50}=8.21 \mathrm{nM}\right)$. Complete inhibition of channel activity (Po $<1 \%$ ) is seen at approximately $100 \mathrm{nM}$.

Concentrations of 84F2 up to $100 \mu \mathrm{M}$ did not result in any return of RyR2 activity.

Following complete inhibition of the channel, addition of $1 \mathrm{mM}$ ATP $(n=5)$, did not show any increase in channel activity. 
A

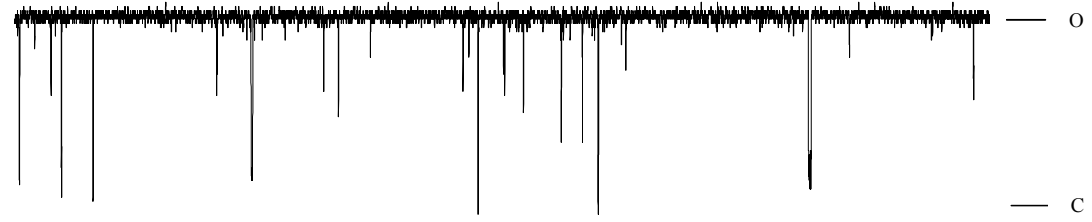

B

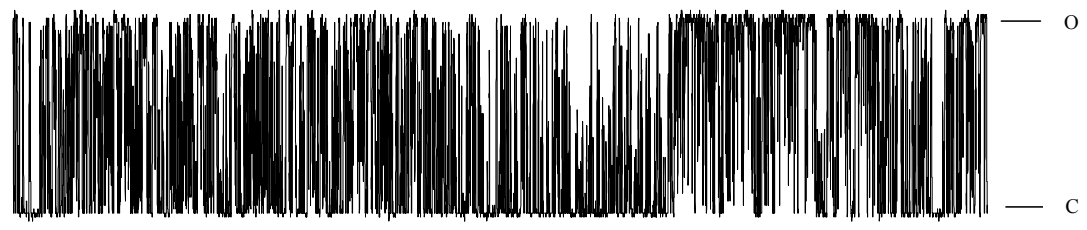

c

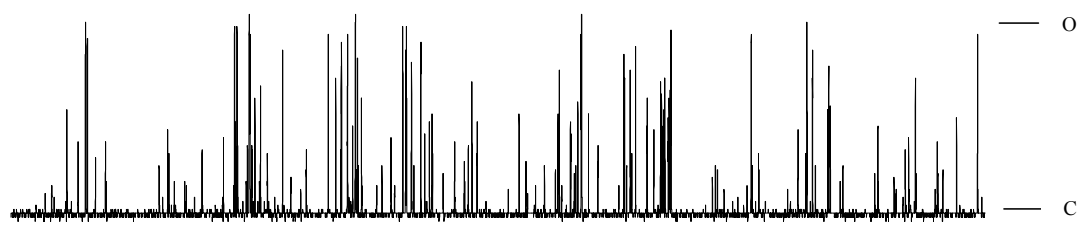

D

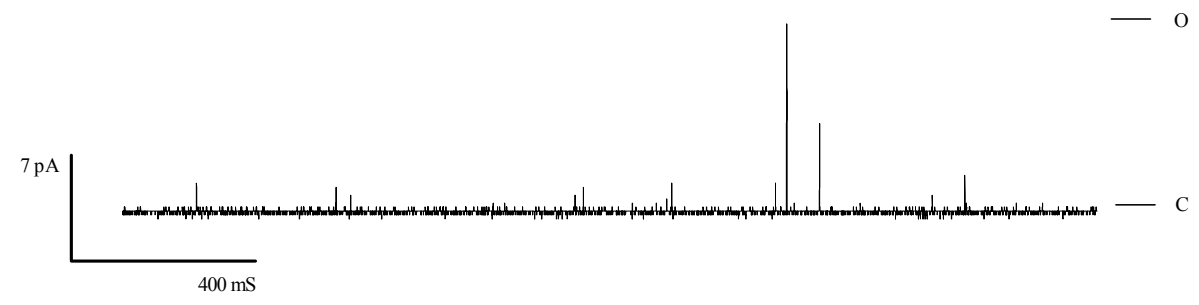

Figure 4.1 Purified RyR2 Single Channel Traces in the Presence of 84F2

Representative single channel recordings of $84 \mathrm{~F} 2$ tested on purified RyR2 reconstituted into Proteoliopsomes. A) Control recording with $25 \mu \mathrm{M} \mathrm{CaCl}_{2}$ and no $84 \mathrm{~F} 2$ present, Po $=0.97$. B) Recording after addition of $3 \mathrm{nM} 84 \mathrm{~F} 2$, Po $=0.43$. C) Recording after the addition of $5 \mathrm{nM} 84 \mathrm{~F} 2, \mathrm{Po}=0.28$. D) Recording after the addition of $35 \mathrm{nM} 84 \mathrm{~F} 2, \mathrm{Po}=0.05$. All traces are shown as two second sections of recordings of at least two minutes. $+36 \mathrm{mV}$ was applied relative to the TRANS chamber indicating channel openings upward as denoted by "o" in the plots. 


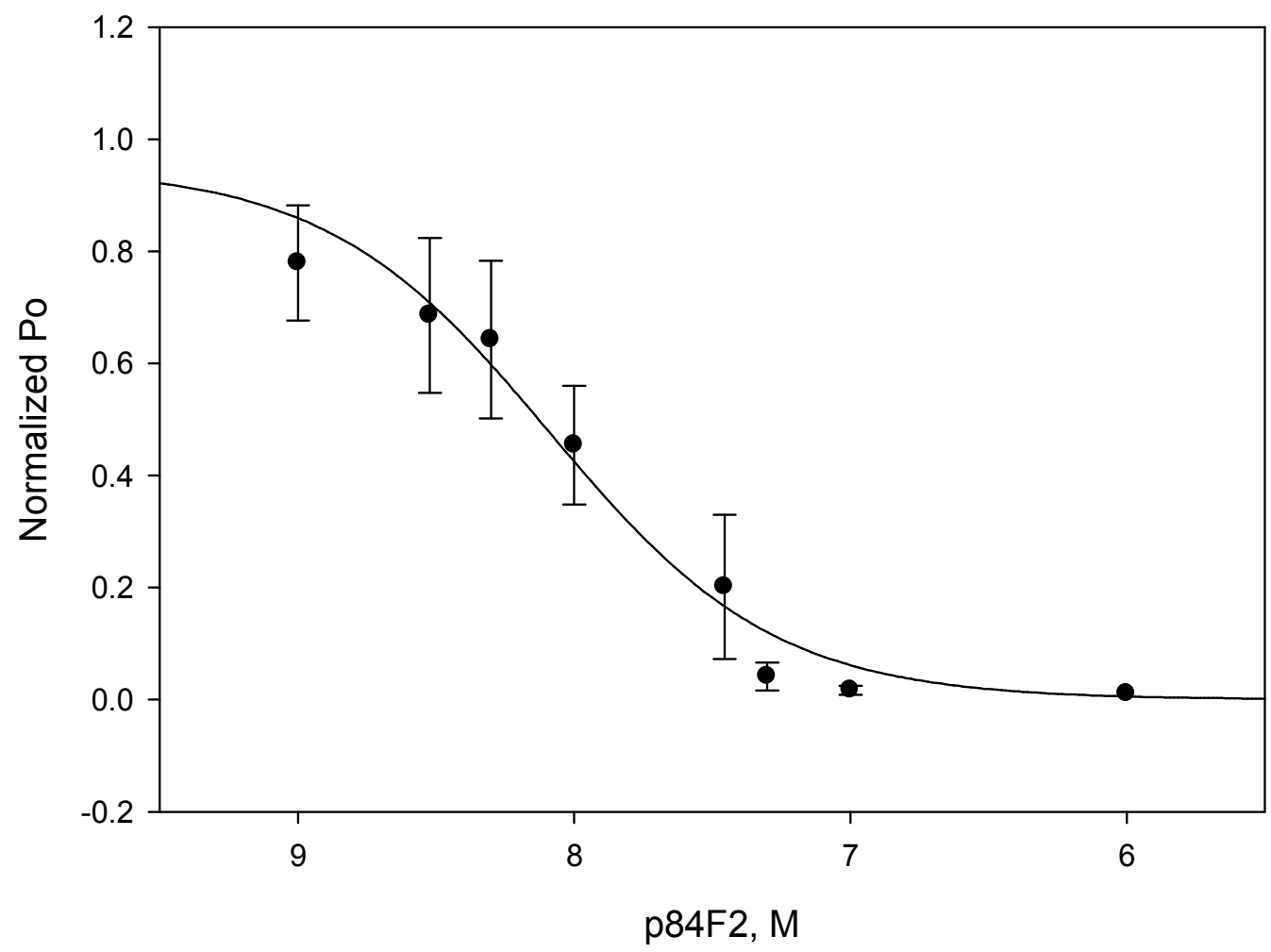

Figure 4.2 84F2 Inhibits Purified RyR2

84F2 inhibits purified RyR2 reconstituted into proteoliposomes from sheep cardiac muscle. Data is normalized as the percent of the control, $25 \mu \mathrm{M}$ calcium single channel recording. Each data point represents 3-12 separate single channel recordings of at least two minutes. Fitting of the curve yields a $\mathrm{pIC}_{50}=8.09 \pm 0.139$ ( \pm standard error), or $\mathrm{IC}_{50}=8.21 \mathrm{nM}$. 


\subsection{Effect of 84F2 on Purified RyR2 Dwell Times}

84F2 single channel data was analyzed to better understand the mechanism by which 84F2 inhibits the gating of RyR2. Average closed and open dwell times were calculated at each concentration of 84F2 tested and significance was calculated (as described in the methods).

Figure 4.3 shows the average closed dwell time (Tc) of RyR2 over a range of 84F2 concentrations ( $1 \mathrm{nM}$ to $100 \mathrm{nM}$ ). The general trend shows an increase in Tc as concentrations are increased. Calculation of $p$-values reveal a significant increase in the closed time over that of the control for concentrations indicated on the graph by "*".

Figure 4.4 shows the average open time (To) of RyR2 for the same data set shown in Figure 4.3. No significant decrease in the open time was seen at lower concentrations of 84F2. At higher concentrations of 84F2, To was decreased in a significant manner as indicated by "*". 


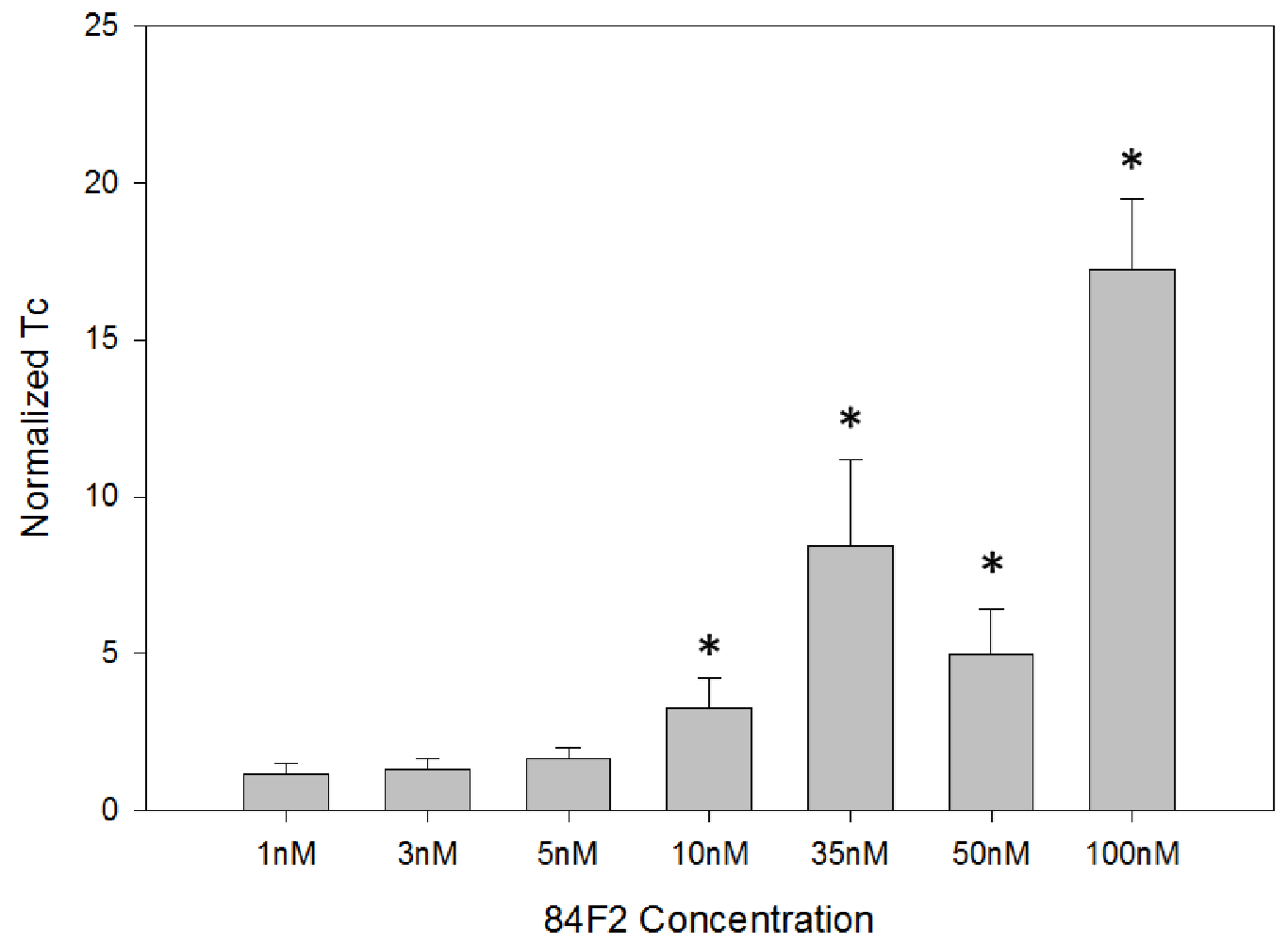

Figure 4.3 84F2 Increases the Closed Dwell Time of Purified RyR2

Calculation of the average closed dwell times of purified RyR2 incorporated into a planar lipid bilayer. Data is normalized as the percent of the control Tc value, measured in the absence of 84F2. Each concentration represents between 3-12 separate single channel recordings. Data which show a significant increase in the closed dwell times compared to the control are indicated by "*." 


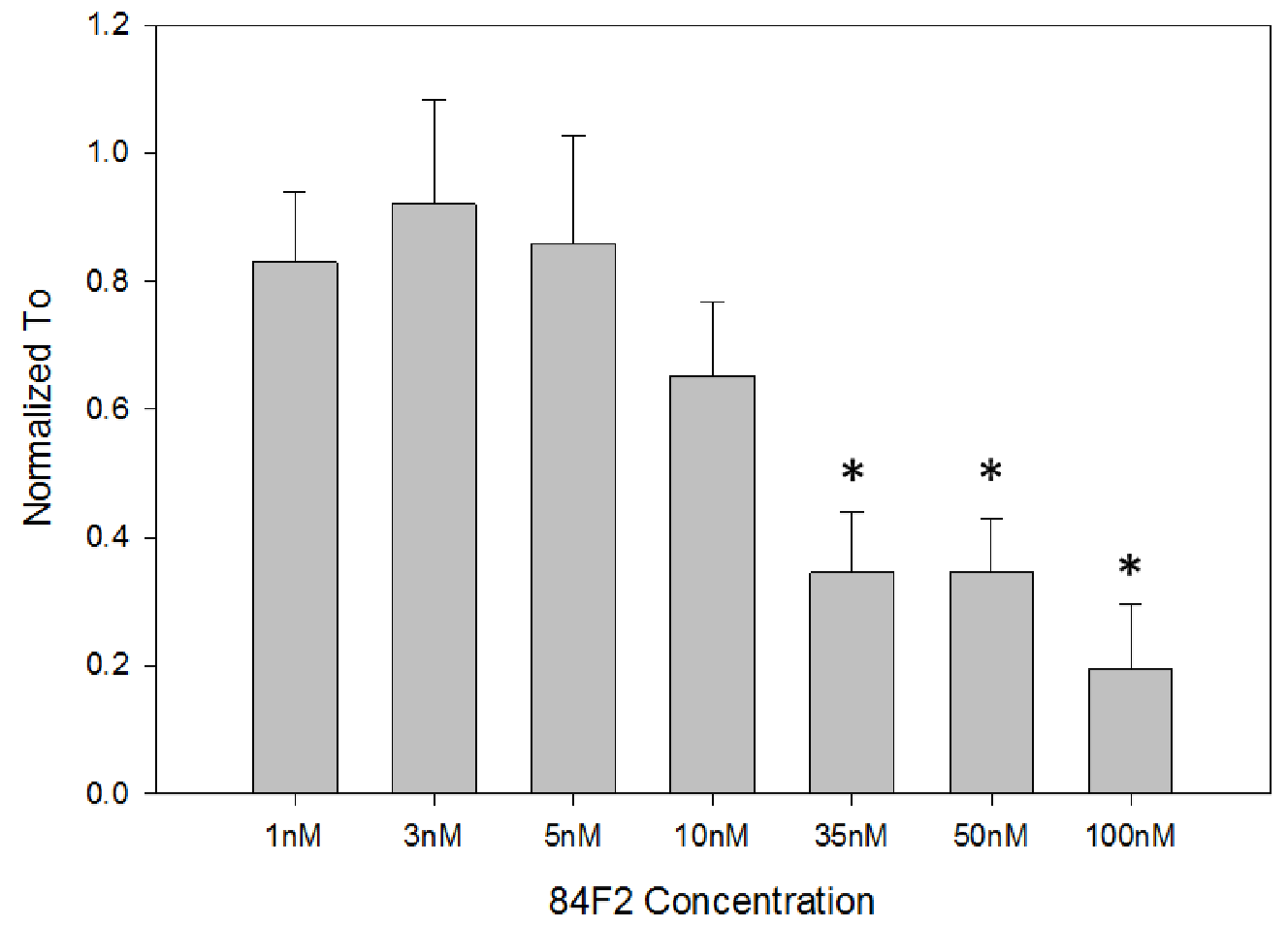

Figure 4.4 84F2 Decreases the Open Dwell Time of Purified RyR2 at High Concentrations

Calculation of the average open dwell times of purified RyR2 incorporated into a planar lipid bilayer. Data is normalized as the percent of the control To value, measured in the absence of 84F2. Each concentration represents between 3-12 separate single channel recordings. Data which show a significant increase in the closed dwell times compared to the control are indicated by "*." 


\subsection{Ionic Strength Dependence of 84F2 Inhibition of Purified RyR2}

$84 F 2$ was tested on purified RyR2 single channel activity at $400 \mathrm{mM} \mathrm{KCl}$ in a manner identical to the experiments in section 4.1. Figure 4.5 shows the dose-response curve of 84F2 added to purified RyR2 at both $250 \mathrm{mM} \mathrm{KCl}$ (closed circles) and $400 \mathrm{mM} \mathrm{KCl}$ (open triangles). $84 \mathrm{~F} 2$ yields $\mathrm{plC} 50$ values of $8.09 \pm 0.14$ and $7.12 \pm 0.16$ at $250 \mathrm{mM} \mathrm{KCl}$ and 400 $\mathrm{mM} \mathrm{KCl}$, respectively $\left(\mathrm{IC}_{50}=8.13 \mathrm{nM}\right.$ and $\left.75.8 \mathrm{nM}\right)$.

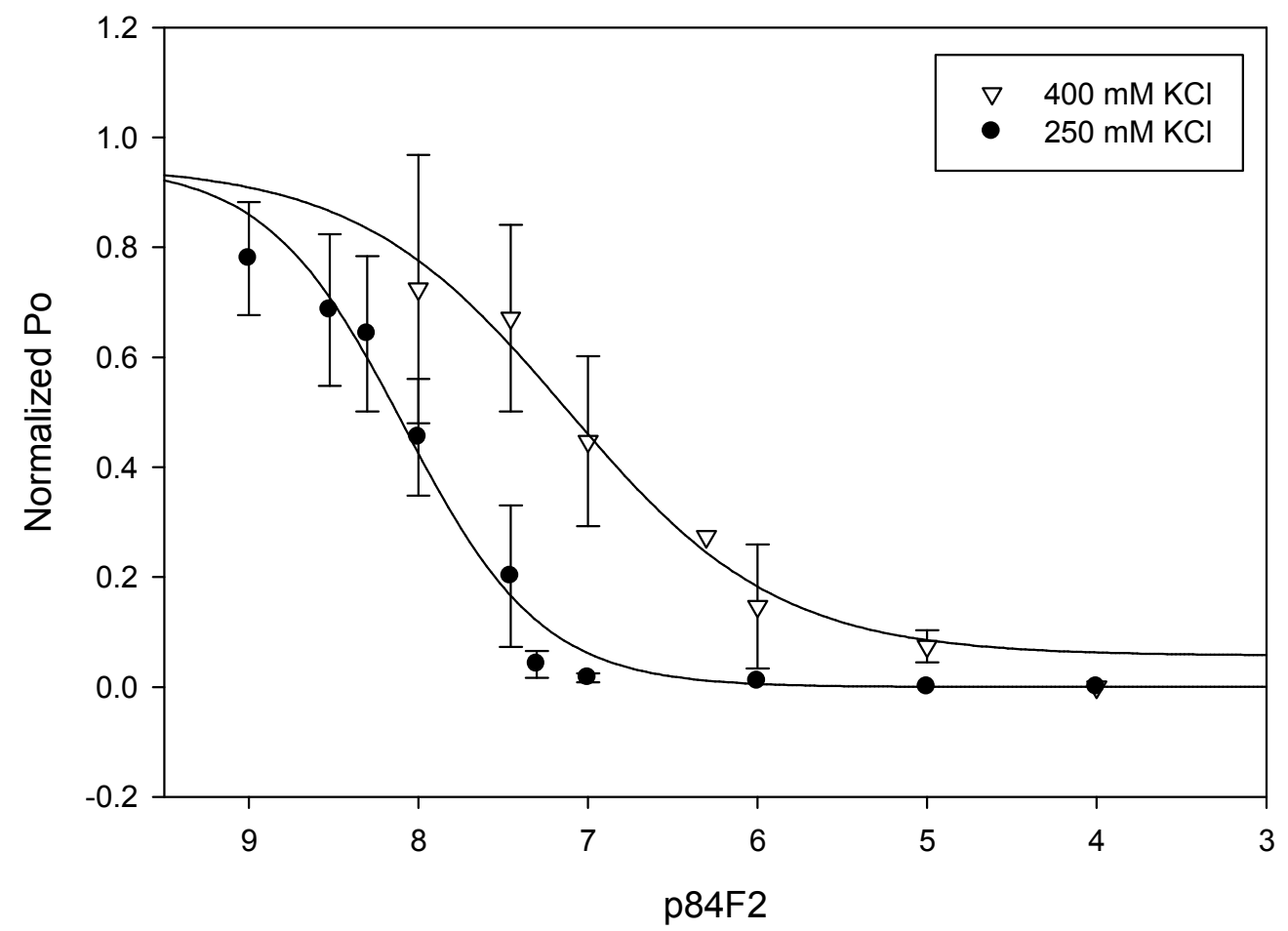

Figure 4.5 84F2 Inhibition of Purified RyR2 at Higher Ionic Strength

$84 F 2$ inhibition of purified RyR2 reconstituted into proteoliposomes. Data is normalized as the percent of the control, $25 \mu \mathrm{M}$ calcium single channel recording. As determined from the fit, $84 \mathrm{~F} 2$ at $250 \mathrm{mM} \mathrm{KCl}$ (closed circles) yields a pIC $50=8.09 \pm 0.14$ ( \pm standard error) $\left(\mathrm{IC}_{50}=8.21 \mathrm{nM}, \mathrm{n}=3-12\right) .84 \mathrm{~F} 2$ at $400 \mathrm{mM}$ $\mathrm{KCl}$ (open triangles) yields a pIC $50=7.12 \pm 0.16$ ( \pm standard error) $\left(\mathrm{IC}_{50}=75.6 \mathrm{nM}, \mathrm{n}=3-8\right)$. 


\subsection{Discussion: 84F2 Directly Inhibits RyR2}

The inhibition of CaSPF in RyR2 ${ }^{\mathrm{R} 1760 /+}$ cardiomyocytes, as well as the inhibition of VT in $\mathrm{RyR}^{\mathrm{R} 176 \mathrm{O} /+}$ mice indicated that $84 \mathrm{~F} 2$ was a potent inhibitor of arrhythmia resulting from RyR2-dysfunction. 84F2 was designed to inhibit RyR2 and the inhibition in CaSPF indicates that RyR2 was inhibited in the presence of $84 \mathrm{~F} 2$. For these reasons, it is believed that 84F2's mechanism of treating arrhythmia is a direct inhibition of RyR2.

Here we have shown that purified RyR2 channels in a planar lipid bilayer were inhibited in the presence 84F2, confirming that 84F2 can directly inhibit RyR2. Table 4.1 summarizes $84 \mathrm{~F} 2$ potency in inhibiting RyR2. The similarity in the potency $\left(\mathrm{IC}_{50}\right)$ of inhibition, in the single channel and $\mathrm{Ca}^{2+}$ spark assays, suggests that $84 \mathrm{~F} 2$ 's inhibition of CaSPF is due to direct inhibition of RyR2. Taken together, these results support the hypothesis that $84 \mathrm{~F} 2$ 's mechanism at treating arrhythmia is through the direct inhibition of $84 \mathrm{~F} 2$.

Table 4.1 Summary of 84F2 Inhibition of RyR2

\begin{tabular}{|l|l|}
\hline Assay & IC50 $(\mathbf{n M})$ \\
\hline Single Channel $(250 \mathrm{mM} \mathrm{KCl})$ & 8.21 \\
\hline Single Channel $(400 \mathrm{mM} \mathrm{KCl})$ & 75.6 \\
\hline $\mathrm{Ca}^{2+}$ Sparks & 35.40 \\
\hline
\end{tabular}

Single channel measurements made at $400 \mathrm{mM} \mathrm{KCl}$ indicated that $84 \mathrm{~F} 2$ has a reduced $\mathrm{IC}_{50}$ at higher ionic strengths. Increases in ionic strength can lead to shielding of charge 
groups, which can lead to weakened interactions. This results suggests the affinity for RyR2 for $84 \mathrm{~F} 2$ is dependent on a charge interaction.

$84 \mathrm{~F} 2$ inhibition was slightly greater at the single channel level $(250 \mathrm{mM} \mathrm{KCl})$ compared to in the $\mathrm{Ca}^{2+}$ spark assay. $\mathrm{IC}_{50}$ values of $8.09 \mathrm{nM}$ and $35.40 \mathrm{nM}$, respectively. These two assays are quite different in nature and small discrepancies in results are expected. However, it is very likely that local concentrations of 84F2 near RyR2 are different in these two assays. In the single channel assay, 84F2 immediately sees the RyR2 cytoplasmic face. 84F2 in the spark assay, first must cross the sarcolemma before interacting with RyR2. Some of the $84 \mathrm{~F} 2$ may be cleaved, prior to, and after entering the cell, thereby reducing concentrations of $84 \mathrm{~F} 2$ around RyR2.

How a compound affects the gating of the channel is important in terms of its usefulness in drug development. Decreasing the open time of the RyR2 results in reduced $\mathrm{Ca}^{2+}$ transient, which can lead to reduced contractile force of muscles. An increased closed time allows for more $\mathrm{Ca}^{2+}$ to be pumped into the SR during diastole, preventing SR $\mathrm{Ca}^{2+}$ load from diminishing. For this reason, ideal compounds to treat RyR2-dysfunction would inhibit RyR2 by increasing the closed time.

Analysis of the single channel dwell times in the presence of $84 \mathrm{~F} 2$ revealed that decreased Po results mainly from a significant increase of RyR2 closed time (Figure 4.3 and Figure 4.4). At high concentration of $84 \mathrm{~F} 2$, the open time was also seen to be significantly decreased. 


\section{Results: Determination of the Active Metabolite of 84F2}

$84 \mathrm{~F} 2$, can be rapidly cleaved at its ester linkage, in either the cell or in the extracellular space. Results at the whole animal level showed that i.p. injection of $84 \mathrm{~F} 2$ reproducibly prevents the incidence of arrhythmia in RyR2 ${ }^{\mathrm{R} 176 \mathrm{O} /+}$ mice for up to 15 minutes after injection. This could indicate that 84F2's reduction in the incidence of VT may be through some other mechanism, besides direct inhibition of RyR2.

This possibility seems remote given that $84 \mathrm{~F} 2$ inhibits RyR2 at similar concentrations the single channel and cellular level, which supported that 84F2's mechanism is through direct inhibition of RyR2. In addition, cellular data showed that 78F3 also inhibits CaSPF from RyR2 ${ }^{\mathrm{R} 1760 /+}$ mouse cardiomyocytes. This all seems to indicate that the cleavage of 84F2 results in a metabolite which may also inhibit RyR2 in a manner like 84F2.

Hypothesis 2: Following breakdown of $84 \mathrm{~F} 2$, either metabolite of $84 \mathrm{~F} 2$ may also inhibit RyR2.

To test this hypothesis, we used purified RyR2 single channels in a planar lipid bilayer to test the compounds, DEAE and 78F3, to determine if either inhibited RyR2 in a manner similar to $84 \mathrm{~F} 2$. 


\subsection{2-(Diethylamino)ethanol (DEAE) Inhibition of Purified RyR2 at the Single Channel}

Level

RyR2, purified and reconstituted into proteoliposomes as described in the methods, was incorporated into an artificial bilayer and single channel activity was tested as a function of DEAE concentration.

Figure 5.1 shows representative single channel recordings of purified RyR2 reconstituted into proteoliposomes in the presence of DEAE and $250 \mathrm{mM} \mathrm{KCl}$ on both sides of the bilayer. Trace A shows a control recording with no DEAE. This control recording yielded an average open probability of 0.70 . Subsequent recordings $B, C$, and D show channels in the presence of $100 \mathrm{nM}, 1 \mu \mathrm{M}$, and $10 \mu \mathrm{M}$ DEAE which resulted in open probabilities of $0.67,0.77$, and 0.76 , respectively.

Further channels were looked at under identical conditions over a range of DEAE concentrations and a dose-response curve was generated. Figure 5.2 shows the doseresponse curve for DEAE inhibition of purified RyR2 at the single channel level. Concentrations of DEAE were added ranging from $10 \mathrm{nM}$ to $10 \mu \mathrm{M}$ and fit as described in the methods. At no concentration of DEAE tested (up to $100 \mu \mathrm{M}$ ) was inhibition seen. 
A

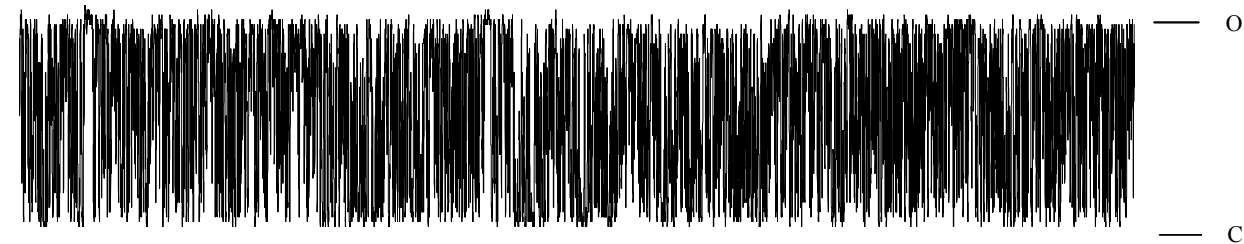

B

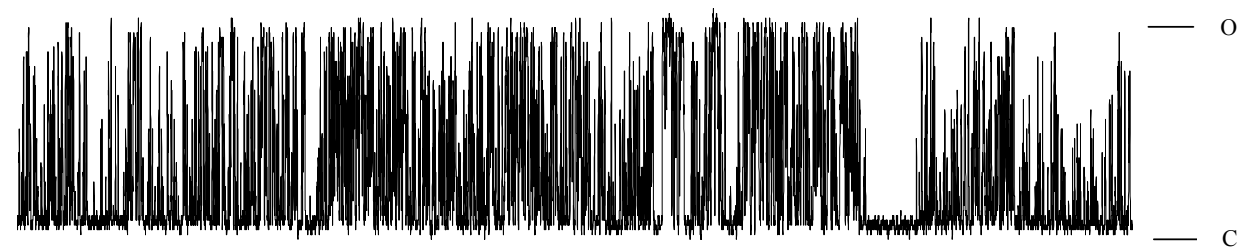

C
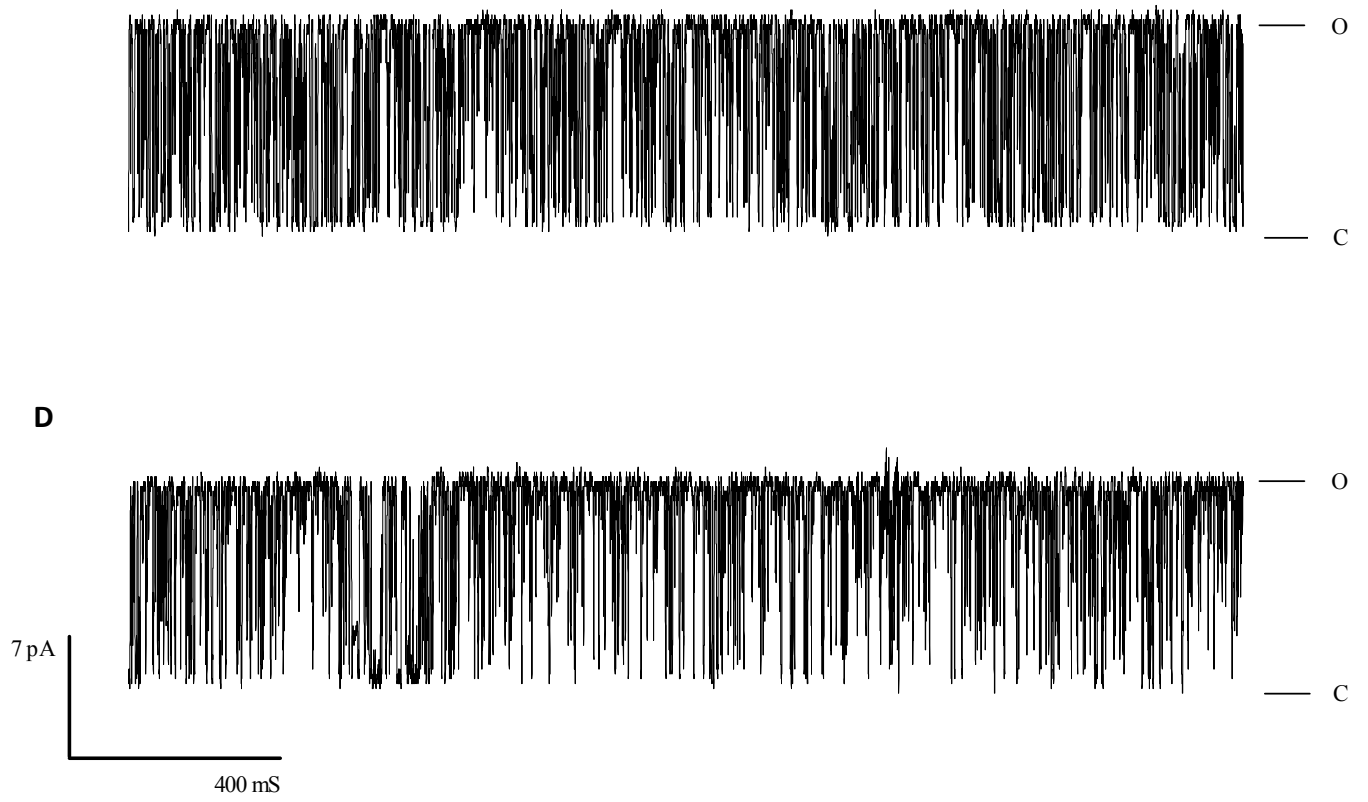

Figure 5.1 Purified RyR2 Single Channel Traces in the Presence of DEAE

Representative single channel recordings of DEAE's effect on purified RyR2 A) Control recording with 25 $\mu \mathrm{M} \mathrm{CaCl} 2$ and no DEAE present, Po $=0.70$. B) Recording after addition of $100 \mathrm{nM} \mathrm{DEAE}, \mathrm{Po}=0.67 . \mathrm{C}$ ) Recording after the addition of $1 \mu \mathrm{M}$ DEAE, Po $=0.77$. D) Recording after the addition $10 \mu \mathrm{M}$ DEAE, Po $=$ 0.76 . All traces are shown as two second sections of recordings of at least two minutes. $+36 \mathrm{mV}$ was applied relative to the TRANS chamber indicating channel openings upward as denoted by " $\mathrm{o}$ " in the plots. 


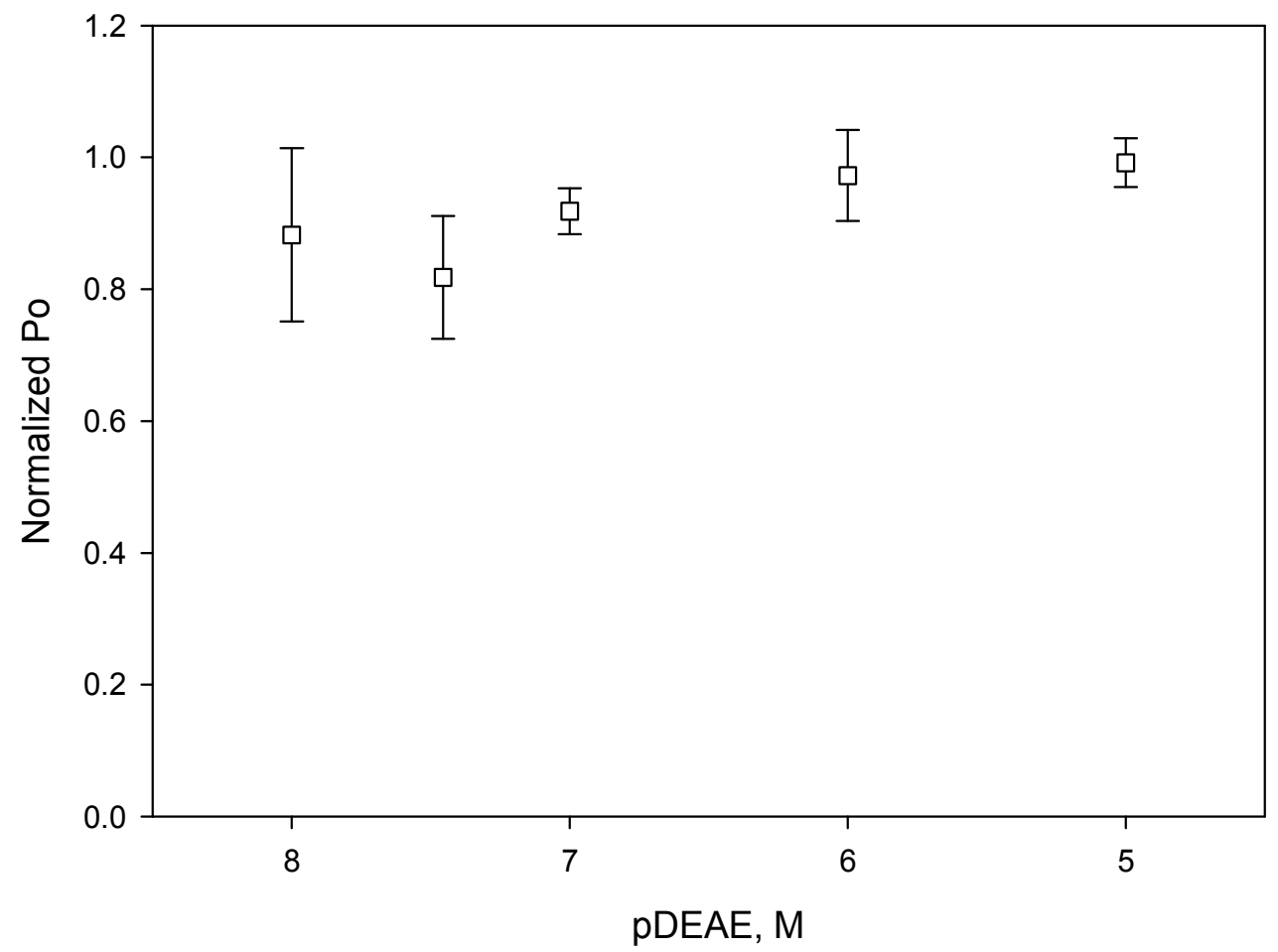

Figure 5.2 DEAE Does Not Inhibit Purified RyR2

DEAE does not inhibit purified RyR2 reconstituted into proteoliposomes. Data is normalized as the percent of the control, $25 \mu \mathrm{M}$ calcium, single channel recording. Each data point represents 3-5 separate single channel recordings of at least two minutes. 


\subsection{F3 Inhibition of Purified RyR2 at the Single Channel Level}

RyR2, purified and reconstituted into proteoliposomes as described in the methods, was incorporated into an artificial bilayer and single channel activity was tested as a function of 78F3 concentration.

Figure 5.3 shows representative single channel recordings of purified RyR2 in the presence of $78 \mathrm{~F} 3$ and $250 \mathrm{mM} \mathrm{KCl}$. Trace A shows a control recording with no $78 \mathrm{~F} 3$, this control recording yielded an average open probability of 0.98 . Subsequent recordings $B$, $\mathrm{C}$, and $\mathrm{D}$ show channels in the presence of $10 \mathrm{nM}, 35 \mathrm{nM}$, and $50 \mathrm{nM} 78 \mathrm{~F} 3$ result in open probabilities of $0.78,0.03$, and 0.003 , respectively. Subsequent recordings on this channel at higher 78F3 concentrations completely inhibited all channel activity. 78F3 is seen here to inhibit purified RyR2 in a concentration-dependent manner.

Further channels were looked at under identical conditions over a range of $78 \mathrm{~F} 3$ concentrations and a dose-response curve was generated. Figure 5.4 shows the doseresponse curve for 78F3 inhibition of purified RyR2 at the single channel level. Concentrations of 78F3 were added ranging from $1 \mathrm{nM}$ to $100 \mu \mathrm{M}$ and the doseresponse curve was fit as described in the methods. The $\mathrm{pIC}_{50}$ of $78 \mathrm{~F} 3$ is $8.02 \pm 0.10\left(\mathrm{IC}_{50}\right.$ $=9.45 \mathrm{nM})$. Complete inhibition of the channel is seen at approximately $100 \mathrm{nM}$. The addition of concentrations of 78F3 up to $100 \mu \mathrm{M}$ resulted in no return of RyR2 activity. 
A

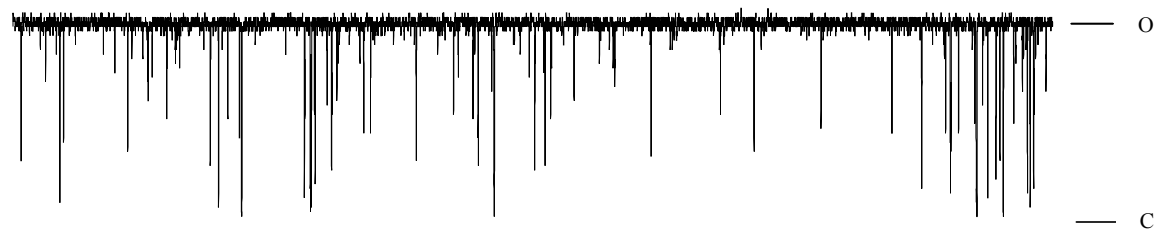

B

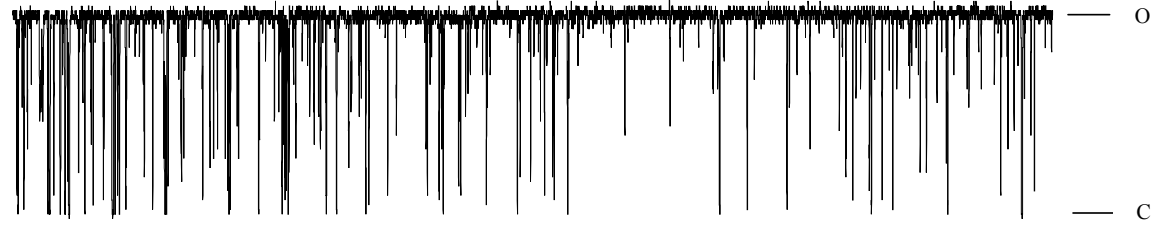

C

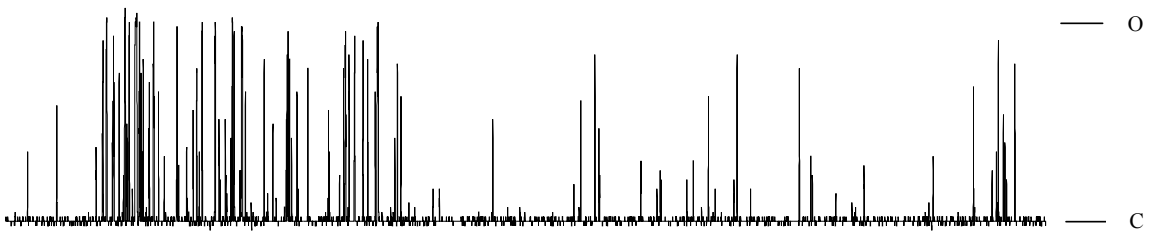

D

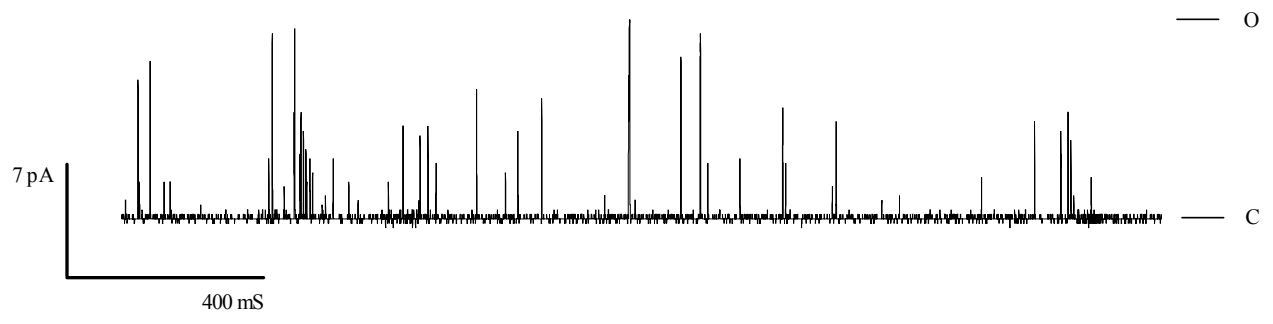

Figure 5.3 Purified RyR2 Single Channel Traces in the Presence of 78F3

Figure Representative single channel recordings of 78F3's effect on purified RyR2. A) Control recording with $25 \mu \mathrm{M} \mathrm{CaCl}_{2}$ and no 78F3 present, $\mathrm{Po}=0.98$. B) Recording after addition of $\left.10 \mathrm{nM} 78 \mathrm{~F} 3, \mathrm{Po}=0.78 . \mathrm{C}\right)$ Recording after the addition of $35 \mathrm{nM} 78 \mathrm{F3}, \mathrm{Po}=0.03$. D) Recording after the addition of $50 \mathrm{nM} 78 \mathrm{F3}, \mathrm{Po}$ $=0.003$. All traces are shown as two second sections of recordings of at least two minutes. $+36 \mathrm{mV}$ was applied relative to the TRANS chamber, indicating channel openings upward as denoted by "o" in the plots. 


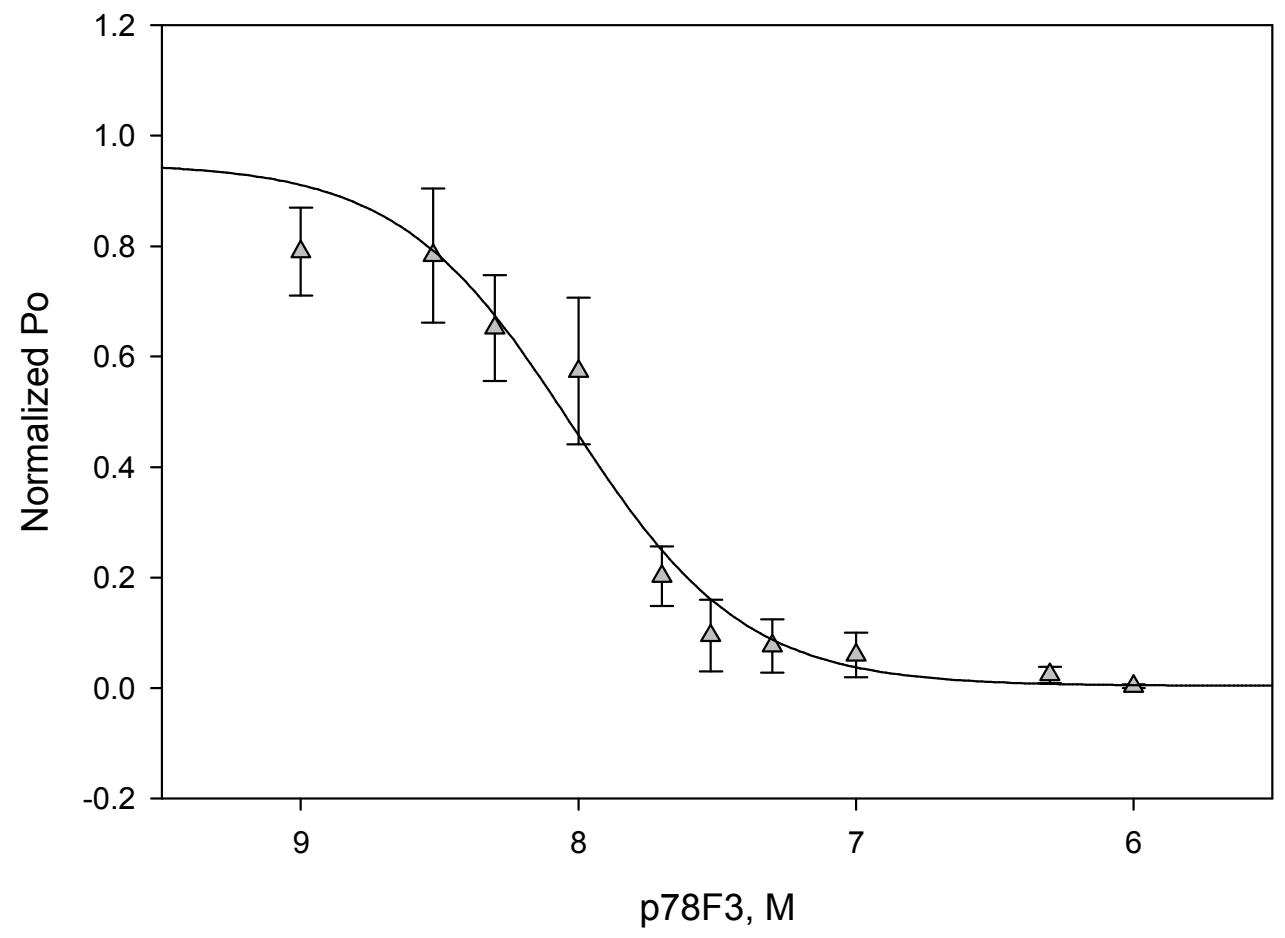

Figure 5.4 78F3 Inhibits Purified RyR2

$78 \mathrm{~F} 3$ inhibits purified RyR2 reconstituted into proteoliposomes. Data is normalized as the percent of the control, $25 \mu \mathrm{M}$ calcium, single channel recording. Each data point represents 3-12 separate single channel recordings of at least two minutes. Determined from the fit, $78 \mathrm{~F} 3$ yields a $\mathrm{plC}_{50}=8.02 \pm 0.10$ ( \pm standard error), $\mathrm{IC} \mathrm{C}_{50}=9.45 \mathrm{nM}$. 


\subsection{Discussion: Following Breakdown of 84F2, 78F3 Inhibits RyR2}

The $\mathrm{Ca}^{2+}$-spark and whole animal data from $\mathrm{RyR} 2^{\mathrm{R} 176 \mathrm{Q} /+}$ mice showed that $84 \mathrm{~F} 2$ was effective in reducing arrhythmogenic states. Results at the single channel level supported the hypothesis that 84F2's mechanism at treating arrhythmia is through direct inhibition of RyR2. However, 84F2's ester can be rapidly cleaved in-vivo making the data at the whole animal level difficult to explain if our hypothesis is correct. This contradiction might be explained if one of the metabolites, following the breakdown of 84F2 could also inhibit RyR2.

Figure 5.5 shows dose-response curves for $84 \mathrm{~F} 2$, and its two metabolites following its cleavage. 78F3 and 84F2 shared near identical dose-response curves, while DEAE showed no inhibition of RyR2. The potency $\left(\mathrm{IC}_{50}\right)$ of these three compounds is summarized in Table 5.1. IC 50 values for $84 \mathrm{~F} 2$ and $78 \mathrm{~F} 3$, were again nearly identical. This data supports our hypothesis that one of 84F2's metabolites, can also inhibit RyR2 in a manner similar to 84F2.

Table 5.1 Summary of 84F2, 78F3, and DEAE Inhibition of Purified RyR2

\begin{tabular}{|l|l|}
\hline Compound & IC $\mathbf{5 0}(\mathbf{n M})$ \\
\hline $84 \mathrm{~F} 2$ & 8.21 \\
\hline $78 \mathrm{~F} 3$ & 9.45 \\
\hline DEAE & No inhibition \\
\hline
\end{tabular}


In the $\mathrm{Ca}^{2+}$-spark assay, 84F2 showed an $\mathrm{IC}_{50}=35.40 \mathrm{nM}$, while $78 \mathrm{~F} 3$ showed a reduced $\mathrm{IC}_{50}=200 \mathrm{nM}$. This does not reflect single channel data, which resulted in identical potency of the two compounds.

The difference in potency in the $\mathrm{Ca}^{2+}$-spark assay may be explained by how each compound enters the cell. 84F2's ester allows it to readily cross the sarcolemma. 78F3, likely does cross the membrane as easily, resulting in lower local concentrations near RyR2. 


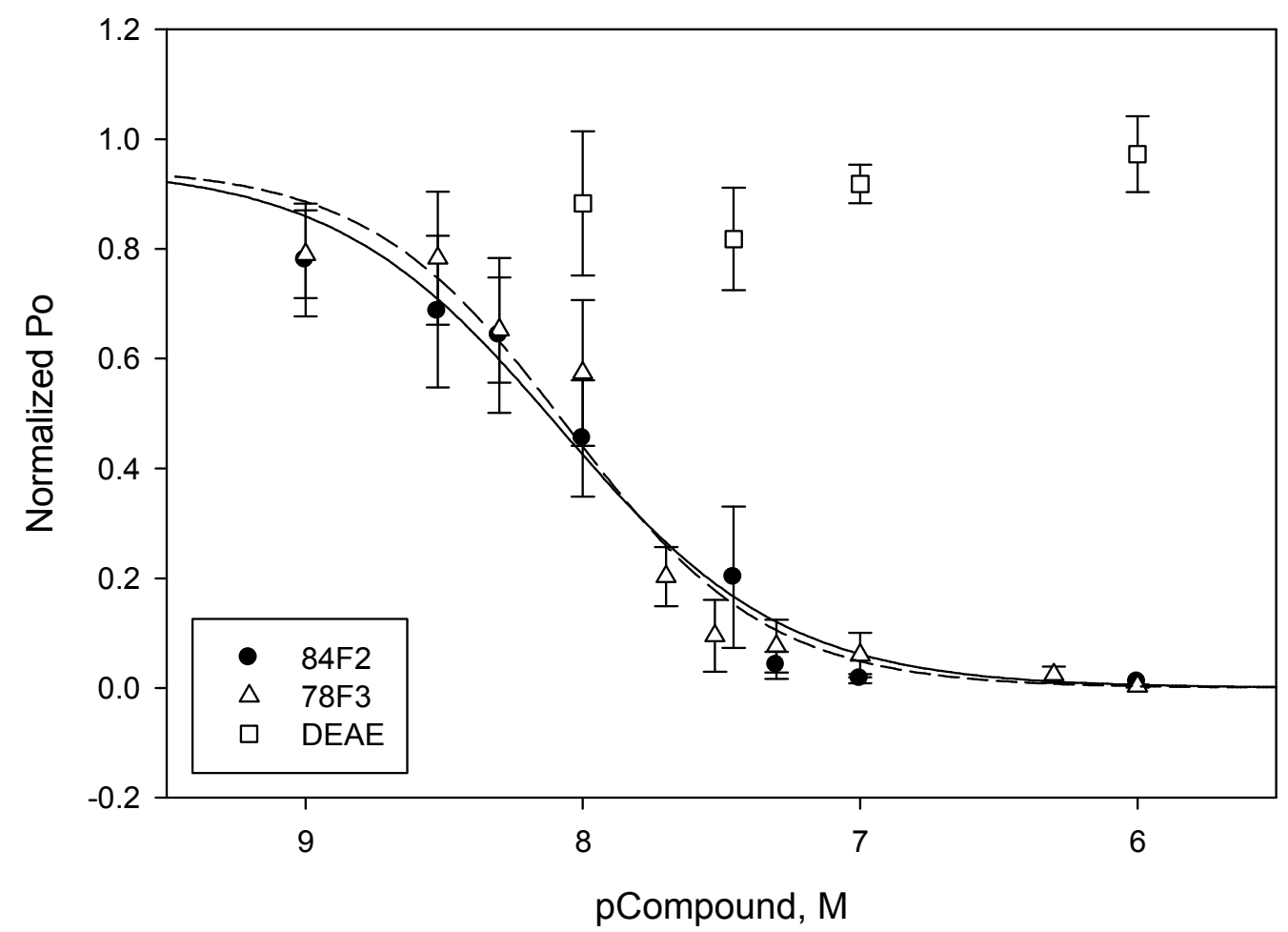

Figure 5.5 84F2, 78F3 and DEAE Inhibition of Purified RyR2

Dose-response curves for 84F2 and its two metabolites, 78F3 and DEAE. Compounds 84F2 (closed circles) and $78 \mathrm{~F} 3$ (open triangles) share near identical dose-responses, with overlapping $\mathrm{plC}_{50}$ values, $8.09 \pm 0.139$ and $8.02 \pm 0.10\left(I C_{50}=8.21 \mathrm{nM}, 9.45 \mathrm{nM}\right)$, respectively. DEAE (open squares) does not show any inhibition of purified RyR2. 


\section{Results: Determination if 84F2 Inhibits RyR2 from Crude Cardiac SR}

Arrhythmogenic states of RyR2 $2^{\mathrm{R} 176 \mathrm{Q} /+}$ cells and RyR2 ${ }^{\mathrm{R} 176 \mathrm{Q} /+}$ which were induced by adrenergic stimulation, were diminished with treatment of 84F2. Normal ECC in these assays was not affected following treatment with 84F2. This suggests that 84F2 may only inhibit RyR2 following its dysfunction, which is known to occur following adrenergic stimulation in CPVT. However, 84F2 was shown at the single channel level to inhibit purified RyR2 in the absence of CPVT mutation or adrenergic stimulation. This suggests an inherent similarity between the purified RyR2 and RyR $2^{\mathrm{R} 1760 /+}$ that undergoes adrenergic stimulation.

The literature suggests that the onset of arrhythmias in CPVT may be due to a dissociation of key regulating proteins from RyR2 during adrenergic stimulation, leading to a leak of $\mathrm{Ca}^{2+}$. In addition, the purification of RyR2 is known to remove associated proteins. This may account for why $84 \mathrm{~F} 2$ can inhibit the purified RyR2, while not affecting normal ECC in RyR2 ${ }^{\mathrm{R} 176 \mathrm{Q} /+}$ animals and cells.

Hypothesis 3: 84F2's inhibition of RyR2 is dependent on the absence of key regulating proteins.

To test this hypothesis, 84F2 was tested on RyR2 from crude cardiac SR which still retains associated proteins. 


\subsection{F2 Single Channel Inhibition of RyR2 from Crude Cardiac SR}

RyR2 from crude cardiac SR vesicles, were incorporated into an artificial bilayer and single channel activity was tested as a function of $84 \mathrm{~F} 2$ concentration. Figure 6.1 shows representative single channel recordings of RyR2 from crude cardiac SR incorporated into a planar bilayer in the presence of $84 \mathrm{~F} 2$. Trace A shows a control recording with no $84 \mathrm{~F} 2$, this control recording yielded an average open probability of 0.95 . Subsequent recordings $B, C$, and $D$ show channels in the presence of $100 \mathrm{nM}, 1 \mu \mathrm{M}$, and $10 \mu \mathrm{M} 84 \mathrm{~F} 2$ resulting in open probabilities of $0.82,0.79$, and 0.52 , respectively. Concentrations tested at $30 \mu \mathrm{M}$ and $100 \mu \mathrm{M}$ showed that complete inhibition of the channel was seen only at a concentration of $100 \mu \mathrm{M}$.

Further channels were examined under identical conditions over a range of 84F2 concentrations and a dose-response curve was generated. Figure 6.2 shows the doseresponse curve for $84 F 2$ inhibition of RyR2 from crude cardiac SR at the single channel level. Concentrations of $84 \mathrm{~F} 2$ ranging from $10 \mathrm{nM}$ to $100 \mu \mathrm{M}$ were added to the $\mathrm{CIS}$ chamber and fit as described in the methods. The pIC 50 of $84 \mathrm{~F} 2$ tested on RyR2 from crude cardiac SR is $5.09 \pm 0.44\left(\mathrm{IC}_{50}=8.11 \mu \mathrm{M}\right)$. Complete inhibition of the channel was seen at $100 \mu \mathrm{M}$ on all channels tested at this concentration $(n=4)$. Each data point on the graph represents between 3 and 7 separate single channel recordings. 
A

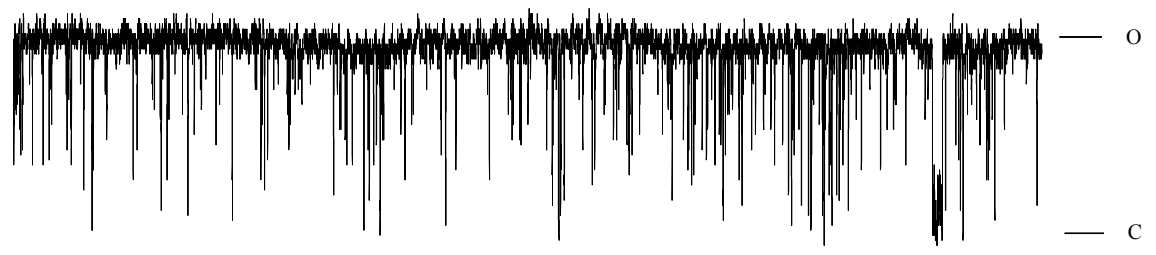

B

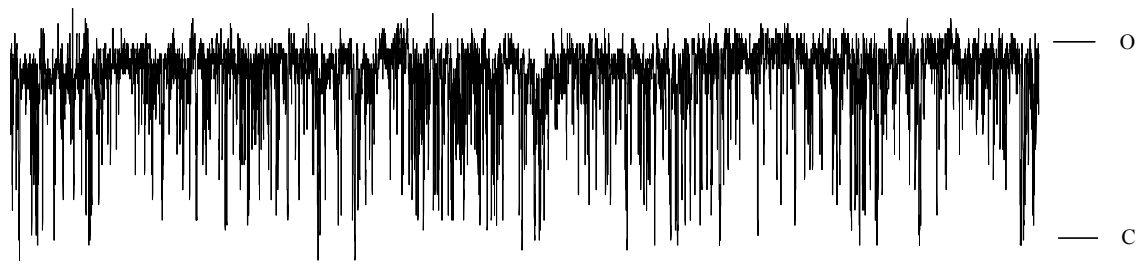

C

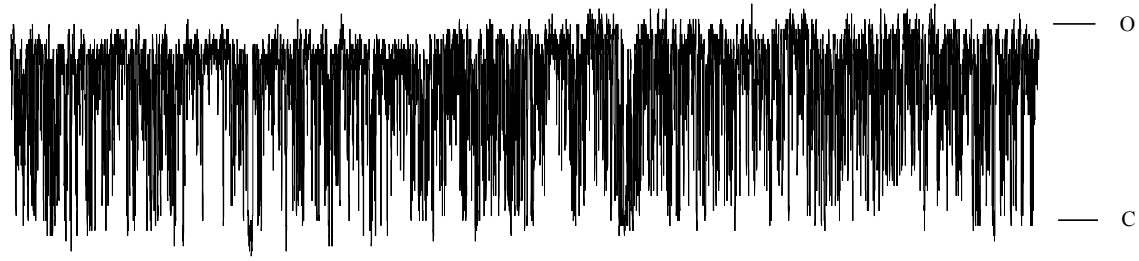

D

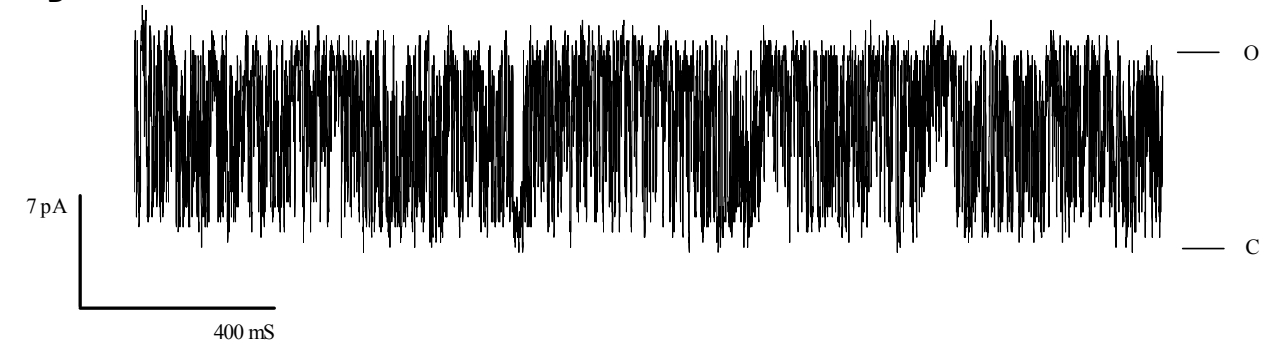

Figure 6.1 Non-Purified RyR2 Single Channel Traces in the Presence of 84F2

Representative single channel recordings of RyR2 from crude cardiac SR in the presence of 84F2. A) Control recording with $25 \mu \mathrm{M} \mathrm{CaCl} 2$ and no $84 \mathrm{~F} 2$ present, $\mathrm{Po}=0.95$. B) Recording after addition of 100 $\mathrm{nM} 84 \mathrm{~F} 2, \mathrm{Po}=0.82$. C) Recording after the addition of $1 \mu \mathrm{M} 84 \mathrm{~F} 2, \mathrm{Po}=0.79$. D) Recording after the addition of $10 \mu \mathrm{M} 84 \mathrm{~F} 2, \mathrm{Po}=0.52$. All traces are shown as two second sections of recordings of at least two minutes. $+36 \mathrm{mV}$ was applied relative to the TRANS chamber, indicating channel openings upward as denoted by "o" in the plots. 


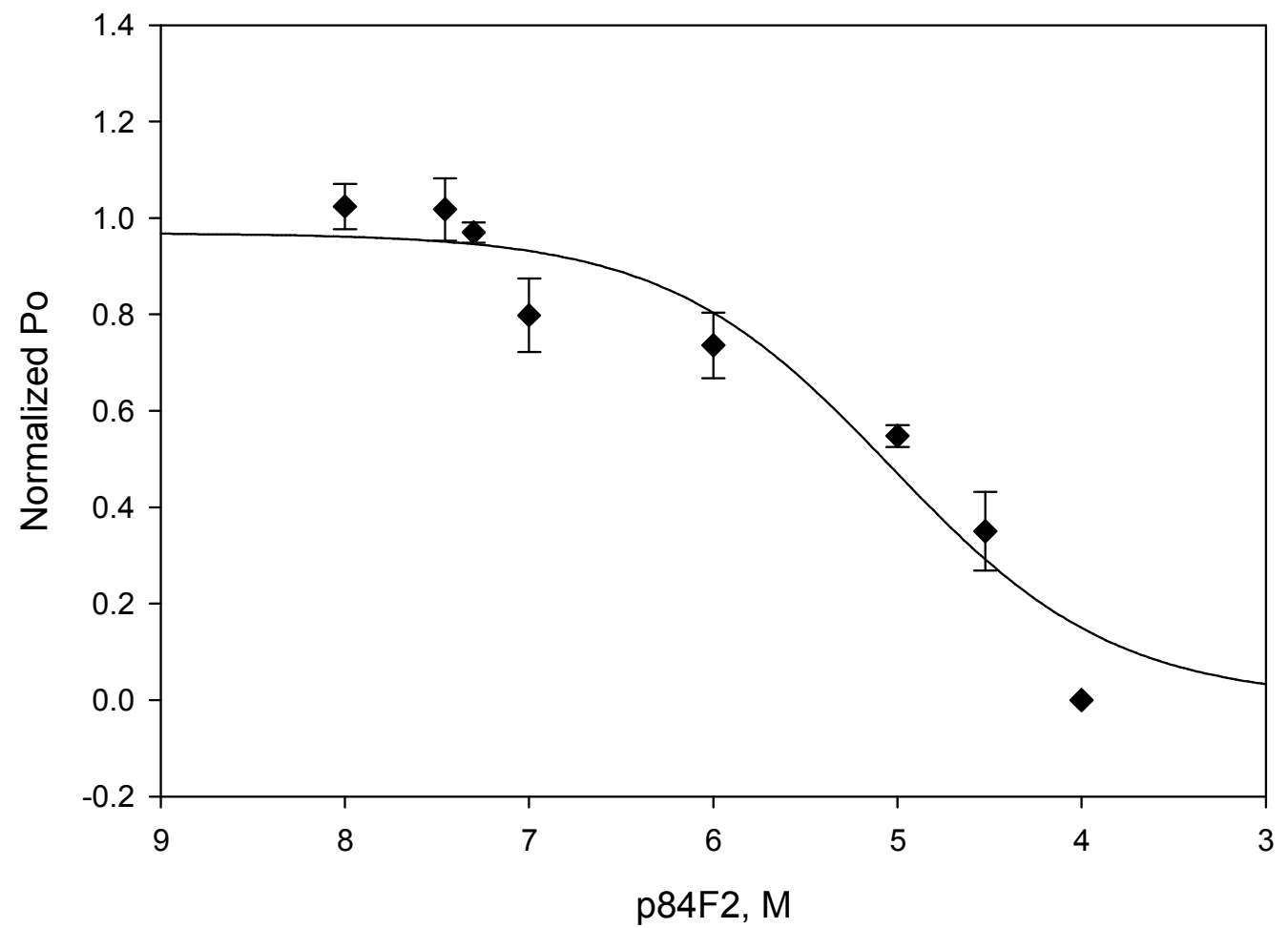

Figure 6.2 84F2 Shows Diminished Inhibition of RyR2 from Crude Cardiac SR

Inhibition of single channel activity on RyR2 from crude cardiac SR in the presence of 84F2. Data is normalized as the percent of the control, $25 \mu \mathrm{M}$ calcium, single channel recording. Each data point represents 3-7 separate single channel recordings of at least two minutes. Fitting of the curve yields a $\mathrm{plC}_{50}=5.09 \pm 0.44$ ( \pm standard error), $\mathrm{IC}_{50}=8.11 \mu \mathrm{M}$. 


\subsection{F2, 78F3, and DEAE Inhibition of Equilibrium ${ }^{3} \mathrm{H}-$ Ryanodine Binding to RyR2 from Crude Cardiac SR}

Equilibrium ${ }^{3} \mathrm{H}$-ryanodine binding to RyR2 from crude cardiac SR was measured in the presence of $84 \mathrm{~F} 2,78 \mathrm{~F} 3$, and DEAE. This assay was carried out in the presence of 250 $\mathrm{mM} \mathrm{KCl}$, at $37^{\circ} \mathrm{C}$ for 3 hours (details described in methods).

Figure 6.3 shows how these compounds affect equilibrium binding. Both $84 \mathrm{~F} 2$ and $78 \mathrm{~F} 3$ showed very weak inhibition of ryanodine binding to RyR2 from crude cardiac SR, with inhibition only occurring at concentrations greater than $10 \mu \mathrm{M}$. DEAE showed no inhibition at concentrations as high as $100 \mu \mathrm{M}$.

Control binding measurements were made at the same time under identical conditions in the absence of any compound. Control equilibrium binding reached a maximum value 1.3 pmol ryanodine / mg SR. Each concentration was repeated in triplicate, normalized as the percent of the control, averaged and standard error was calculated. 


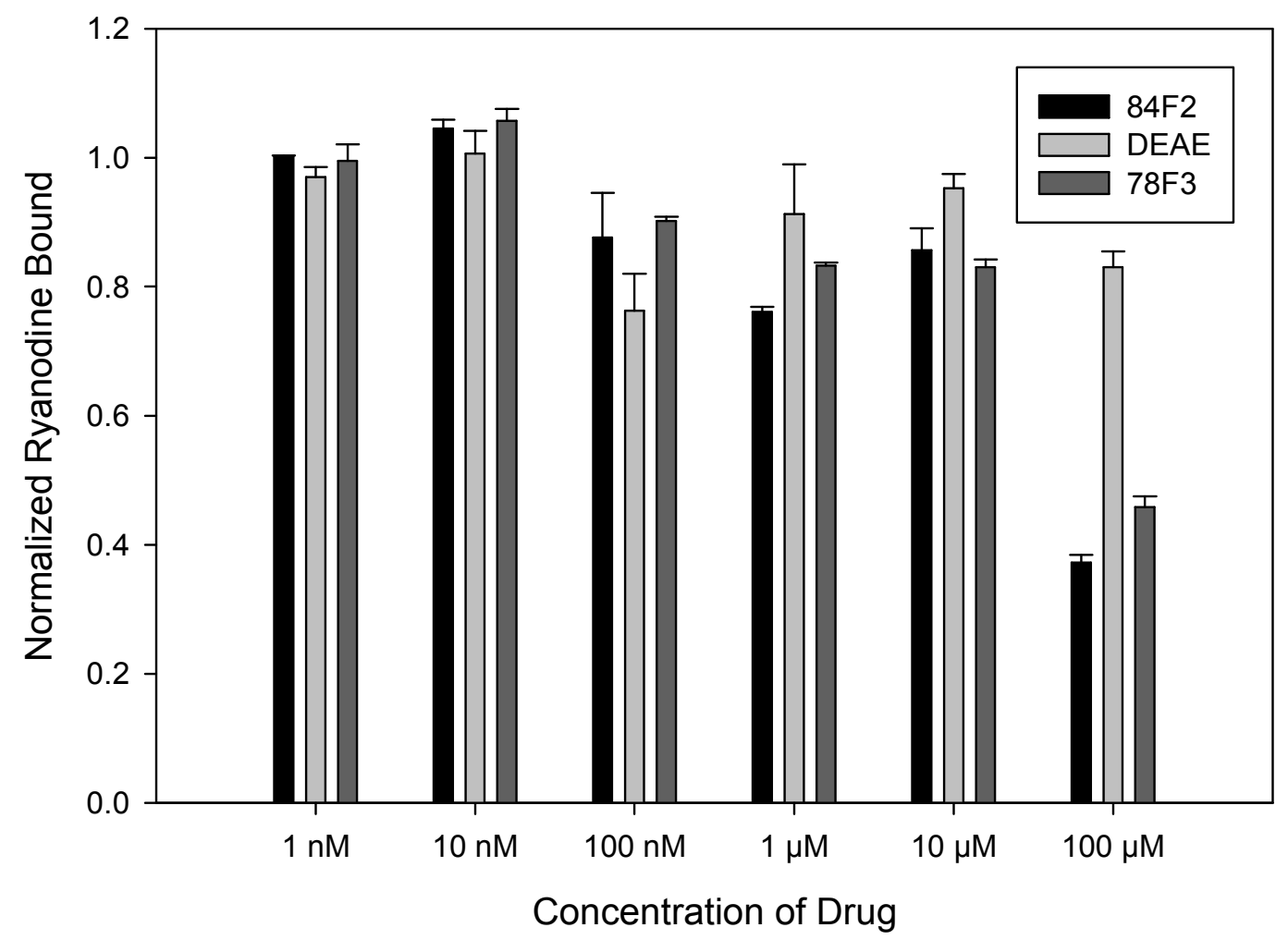

Figure 6.3 Equilibrium ${ }^{3} \mathrm{H}$-Ryanodine Binding in the Presence of 84F2, 78F3, and DEAE

84F2 shows no inhibition of ${ }^{3} \mathrm{H}$-ryanodine binding to crude cardiac SR except at very high concentrations (> $10 \mu \mathrm{M})$. 78F3 shows a similar inhibition to 84F2 and DEAE showed no inhibition at all concentrations tested. Each bar of the graph represents measurements made in triplicate, normalized to the percentage of control, \pm standard error. Control equilibrium binding measured 1.3 pmol ryanodine / mg SR and was normalized to 1 . 


\subsection{Rate of ${ }^{3} \mathrm{H}$-Ryanodine Binding in the Presence of $84 \mathrm{~F} 2$}

Figure 6.4 shows how $84 \mathrm{~F} 2$ affects the rate of ${ }^{3} \mathrm{H}$-ryanodine binding to crude cardiac SR vesicles. 84F2 was tested at concentrations of $0 \mu \mathrm{M}$ (closed circles), $1 \mu \mathrm{M}$ (open circles), $5 \mu \mathrm{M}$ (closed triangles) and $10 \mu \mathrm{M}$ (open triangles). The amount of ${ }^{3} \mathrm{H}$-ryanodine bound was measured at 5, 10, 15, and 20 minutes and fit as described in the methods.

Results, as summarized in Table 6.1, show that $84 \mathrm{~F} 2$ inhibits the rate of ${ }^{3} \mathrm{H}$-ryanodine binding to crude cardiac SR in a concentration dependent manner. An estimate of the concentration of half-maximal inhibition (using 15.53 as the maximal rate and 0 as the minimal rate) yields an $\mathrm{IC}_{50}$ is between $1 \mu \mathrm{M}$ and $5 \mu \mathrm{M} 84 \mathrm{~F} 2$.

Table 6.1 Summary of 84F2 Inhibition in the rate of ${ }^{3} \mathrm{H}$-Ryanodine Binding

\begin{tabular}{|l|l|l|}
\hline Concentration $84 \mathrm{~F} 2(\mu \mathrm{M})$ & $\begin{array}{l}\text { Rate } \pm \text { Standard Error } \\
(\mathrm{fmol} / \mathrm{mg} / \mathrm{min})\end{array}$ & $\begin{array}{l}\text { Y-Intercept } \pm \text { Standard } \\
\text { Error }(\mathrm{fmol} / \mathrm{mg})\end{array}$ \\
\hline 0 & $15.5 \pm 0.66$ & $118.01 \pm 9.05$ \\
\hline 1 & $10.7 \pm 2.02$ & $132.43 \pm 27.65$ \\
\hline 5 & $6.8 \pm 1.69$ & $116.40 \pm 23.09$ \\
\hline 10 & $3.8 \pm 0.48$ & $116.33 \pm 6.62$ \\
\hline
\end{tabular}




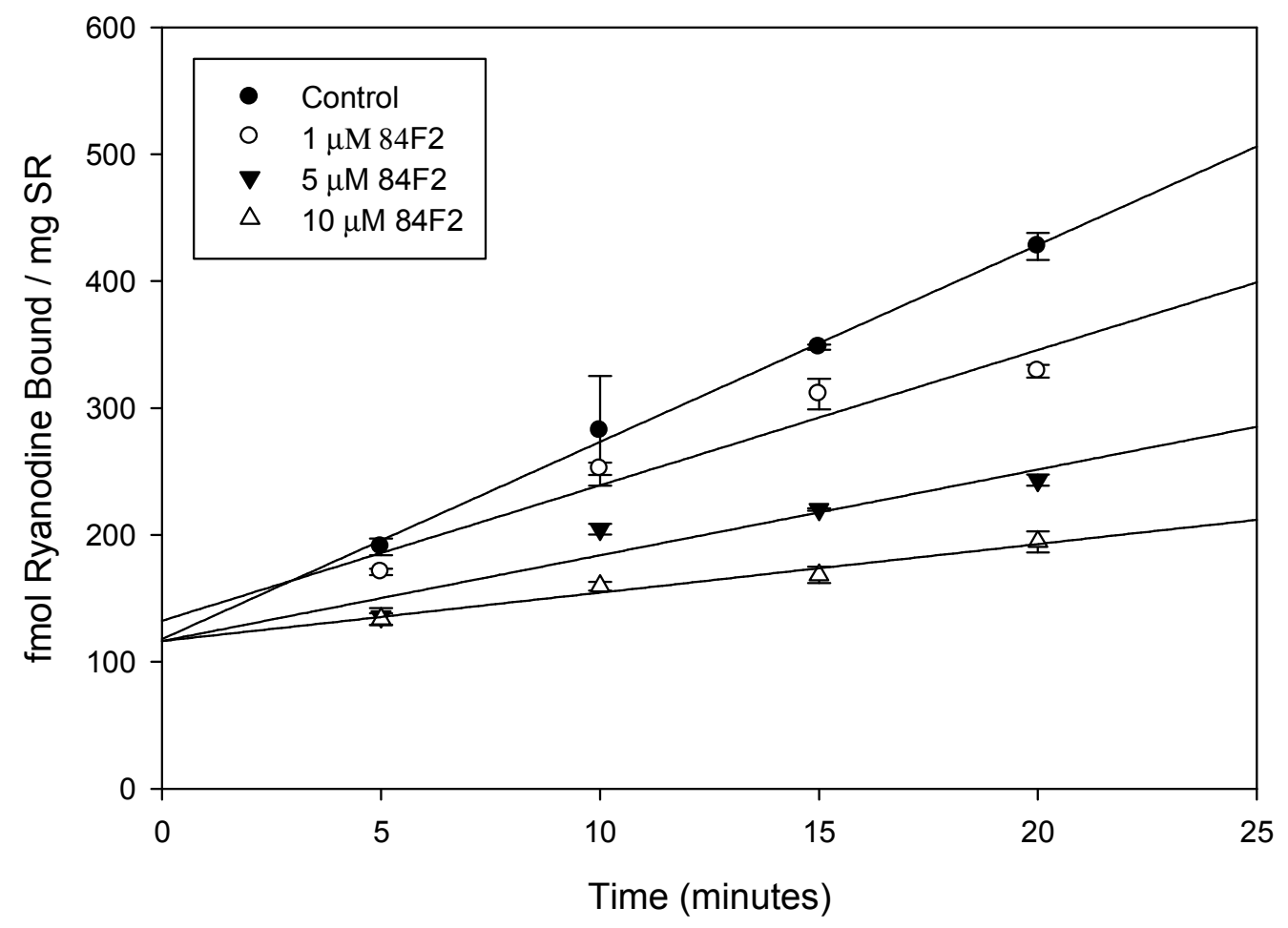

Figure 6.4 84F2 Decreases the Rate of ${ }^{3} \mathrm{H}$-Ryanodine Binding to RyR2 from Crude Cardiac SR

84F2 shows a moderate inhibition in the rate of ${ }^{3} \mathrm{H}$-ryanodine binding to RyR2 from crude cardiac SR. Rates were determined as the slope of the linear fits. Control time points (closed circle) at 5, 10, 15 and 20 minutes yield the maximum rate of $15.5 \mathrm{fmol} / \mathrm{mg} / \mathrm{min} \pm 0.66 \mathrm{fmol} / \mathrm{mg} / \mathrm{min}$. Identical time points were used with $84 \mathrm{~F} 2$ concentrations of $1 \mu \mathrm{M}$ (open circles), $5 \mu \mathrm{M}$ (closed triangles) and $10 \mu \mathrm{M}$ (open triangles). These concentrations yielded rates of $10.7 \mathrm{fmol} / \mathrm{mg} / \mathrm{min} \pm 2.02 \mathrm{fmol} / \mathrm{mg} / \mathrm{min}, 6.8 \mathrm{fmol} / \mathrm{mg} / \mathrm{min} \pm 1.69$ $\mathrm{fmol} / \mathrm{mg} / \mathrm{min}, 3.8 \mathrm{fmol} / \mathrm{mg} / \mathrm{min} \pm 0.48 \mathrm{fmol} / \mathrm{mg} / \mathrm{min}$, respectively. Each Data point is carried out in triplicate and represents the mean \pm standard error. 


\subsection{Discussion: 84F2 has Reduced Inhibition of RyR2 from Crude Cardiac SR}

84F2 has been shown to inhibit arrhythmogenic states resulting from RyR2-dysfunction induced by adrenergic stimulation in a CPVT animal model. 84F2 had not effect on normal ECC in these animals suggesting 84F2 only treated RyR2 in a dysfunctional state. Our single channel results showed that 84F2 inhibited purified RyR2 at similar concentrations to the inhibition of CaSPF in RyR2 ${ }^{\mathrm{R} 176 \mathrm{Q} /+}$ cardiomyocytes. This suggested that purified RyR2 has inherent similarities to RyR2 ${ }^{\mathrm{R} 1760 /+}$ following adrenergic stimulation.

Here we have shown that 84F2 Inhibition of RyR2 from crude cardiac SR was reduced compared to inhibition seen on both the purified RyR2 single channels and CaSPF of RyR2 ${ }^{\mathrm{R} 1760 /+}$ myocytes. Results, as summarized in Table 6.2, support the hypothesis that $84 \mathrm{~F} 2$ is more potent at inhibiting RyR2 in the absence of key regulating proteins.

Table 6.2 Summary of 84F2 Inhibition in the Presence of Associated Proteins

\begin{tabular}{|l|l|l|}
\hline Assay & RyR2 Preparation & IC50 (nM) \\
\hline Single Channel & Purified & 8.21 \\
\hline CaSPF & RyR2 ${ }^{\mathrm{R} 176 \mathrm{Q} /+}$ & 35.40 \\
\hline Single Channel & Crude Cardiac SR & 8,110 \\
\hline $\begin{array}{l}\text { Rate of }{ }^{3} \mathrm{H}-\text { Ryanodine } \\
\text { Binding }\end{array}$ & Crude Cardiac SR & $\sim 1,000-5,000$ \\
\hline $\begin{array}{l}\text { Equilibrium }{ }^{3} \mathrm{H}-\text { Ryanodine } \\
\text { Binding }\end{array}$ & Crude Cardiac SR & $>10,000$ \\
\hline
\end{tabular}


The strongest evidence supporting this hypothesis is the large difference in single channel results as shown in Figure 6.5. Figure 6.5 Comparison of Single Channel Inhibition of RyR2 from Crude Cardiac SR and Purified RyR2 84F2 showed a reduction in inhibition of RyR2 from crude cardiac SR compared to measurements carried out on purified RyR2 under identical conditions. No overlap in inhibition can be seen at any concentration of $84 \mathrm{~F} 2$ and the half-maximal inhibition concentration increased approximately 1,000 fold, from $8.21 \mathrm{nM}$ to $8.11 \mu \mathrm{M}$, when tested on RyR2 from crude cardiac SR. Inhibition in the rate of ${ }^{3} \mathrm{H}$-ryanodine binding is seen at concentrations closely resembling concentrations seen with single channel results of RyR2 from crude cardiac SR.

Equilibrium experiments had differing results, showing a greater reduction in inhibition of RyR2 from crude cardiac SR by 84F2. Only concentrations $>10 \mu \mathrm{M}$ of $84 \mathrm{~F} 2$ were effective in inhibiting binding of ${ }^{3} \mathrm{H}$-ryanodine and complete inhibition was not achievable at concentrations as high as $100 \mu \mathrm{M}$.

The ${ }^{3} \mathrm{H}$-ryanodine binding results indicate that rates more closely reflect experiments carried out at the single channel level. Numerous equilibrium ${ }^{3} \mathrm{H}$-ryanodine binding experiments were carried out with $84 \mathrm{~F} 2$ to try to characterize these differences, however, under no conditions tested was 84F2 inhibition of ${ }^{3} \mathrm{H}$-ryanodine binding altered (Data not shown in this work). Several compounds are known to inhibit RyR2 single channel activity while not inhibiting ${ }^{3} \mathrm{H}$-ryanodine binding, but the cause remains unknown. 
One interesting result from the equilibrium experiments was that, like in the single channel studies, 84F2 and 78F3 shared similar inhibition concentrations of ${ }^{3} \mathrm{H}$-ryanodine equilibrium binding, while DEAE showed no inhibition. This is further evidence that $78 \mathrm{~F} 3$ can also inhibit RyR2 following the breakdown of 84F2. 


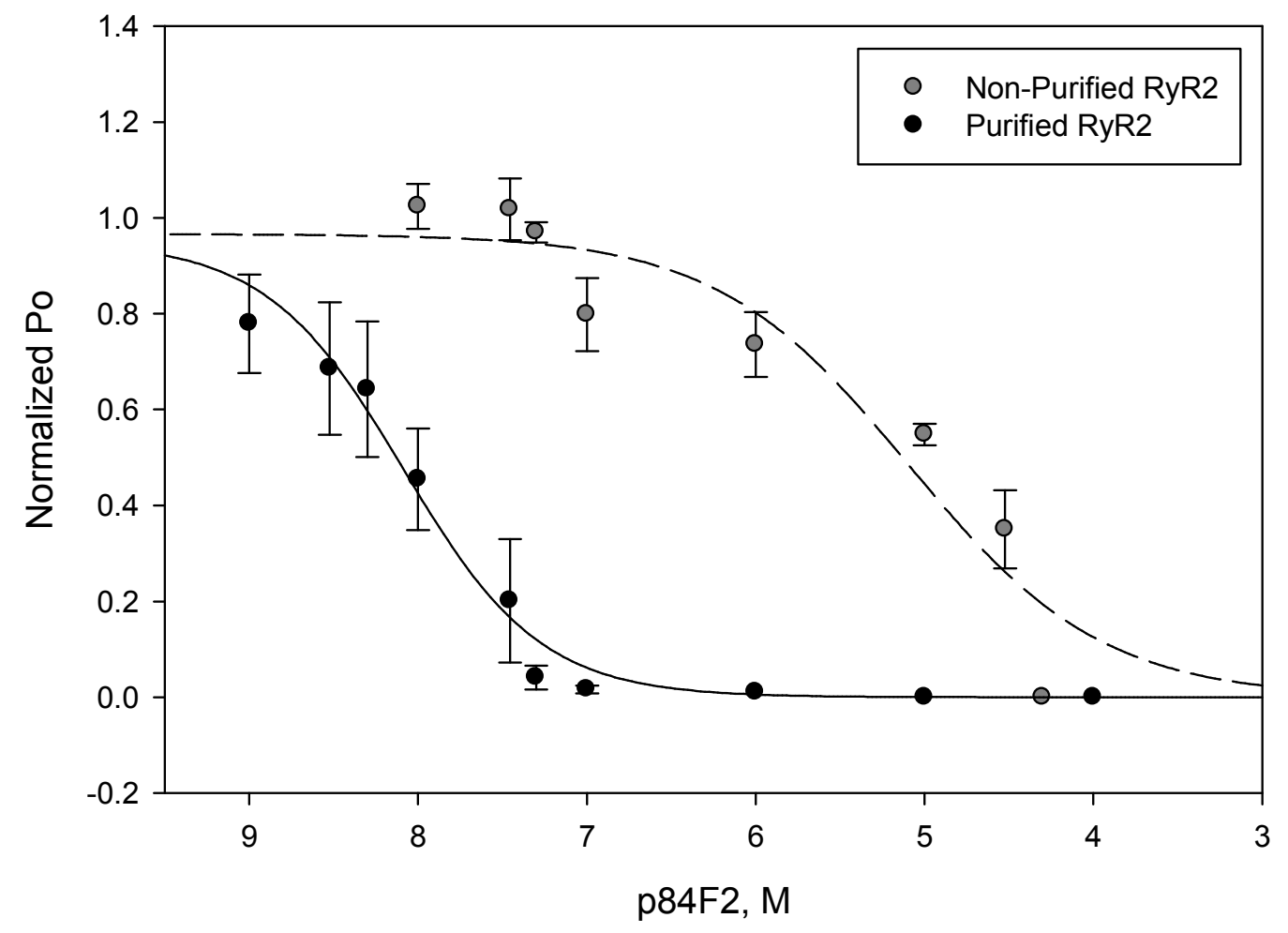

Figure 6.5 Comparison of Single Channel Inhibition of RyR2 from Crude Cardiac SR and Purified RyR2 in the Presence of 84F2

Dose-response curves for 84F2 inhibition of purified RyR2 (black circles) and non-purified RyR2 (grey circles). 84F2 shows clear differences in its strength of inhibition of purified and non-purified RyR2, yielding $\mathrm{IC}_{50}$ values of $8.13 \mathrm{nM}$ and $8.11 \mu \mathrm{M}$, respectively. 


\section{Results: Determination of Calmodulin's Role in 84F2's Inhibition of RyR2}

The ability of 84F2 to treat arrhythmogenic states in CPVT animals, while not affecting normal ECC, was hypothesized to be due to 84F2 only inhibiting RyR2 in a dysfunctional state. In support of this hypothesis, $84 \mathrm{~F} 2$ was a significantly less potent inhibitor of RyR2 in crude cardiac SR (in the presence of associated proteins) than in our purified preparation.

Particularly interesting is the similarity between the concentrations of $84 \mathrm{~F} 2$ that inhibit the purified RyR2 at the single channel level, and inhibit CaSpF in RyR2 ${ }^{\mathrm{R} 1760 /+}$ cardiomyocytes. This indicates that the purified RyR2 is in a conformation similar to dysfunctional RyR2 which arises from adrenergic stimulation of CPVT cells.

Evidence in the literature suggests that RyR2-dysfunction in CPVT is due to a dissociation of CaM from RyR2 during adrenergic stimulation ${ }^{83,84,85}$. CaM is also known to be absent from RyR2 following the purification protocols we follow.

Hypothesis 4: Inhibition of RyR2 by $84 \mathrm{~F} 2$ is dependent on CaM dissociation from RyR2. To test this hypothesis, we compared the sensitivity of the purified RyR2 at the single level to inhibition by $84 \mathrm{~F} 2$ in the absence and presence of CaM. 


\subsection{Inhibition of Purified RyR2 Single Channel Activity by $84 \mathrm{~F} 2$ in the Presence of Calmodulin}

Inhibition of purified RyR2 by $84 \mathrm{~F} 2$ was measured in a manner identical to the experiments in Chapter 4, with CaM added to the CIS chamber prior to the addition of 84F2. Following the addition CaM, increasing concentrations of $84 \mathrm{~F} 2$ were added to the CIS chamber and single channel activity was recorded.

Figure 7.1 shows the dose-response curve for $84 \mathrm{~F} 2$ inhibition of RyR2 in the presence of either 100 nM CaM (open circles), or 1 MM CaM (closed circles). 84F2 has nearly an identical dose-response curve at both concentrations of CaM. 84F2 in the presence of 1 $\mu \mathrm{M}$ CaM resulted in a $\mathrm{plC}_{50}=5.75 \pm 0.39\left(\mathrm{IC}_{50}=1.77 \mu \mathrm{M}\right) .84 \mathrm{~F} 2$ in the presence of $100 \mathrm{nM}$ CaM resulted in a pIC $50=5.73 \pm 0.29\left(\mathrm{IC}_{50}=1.86 \mu \mathrm{M}\right)$. 


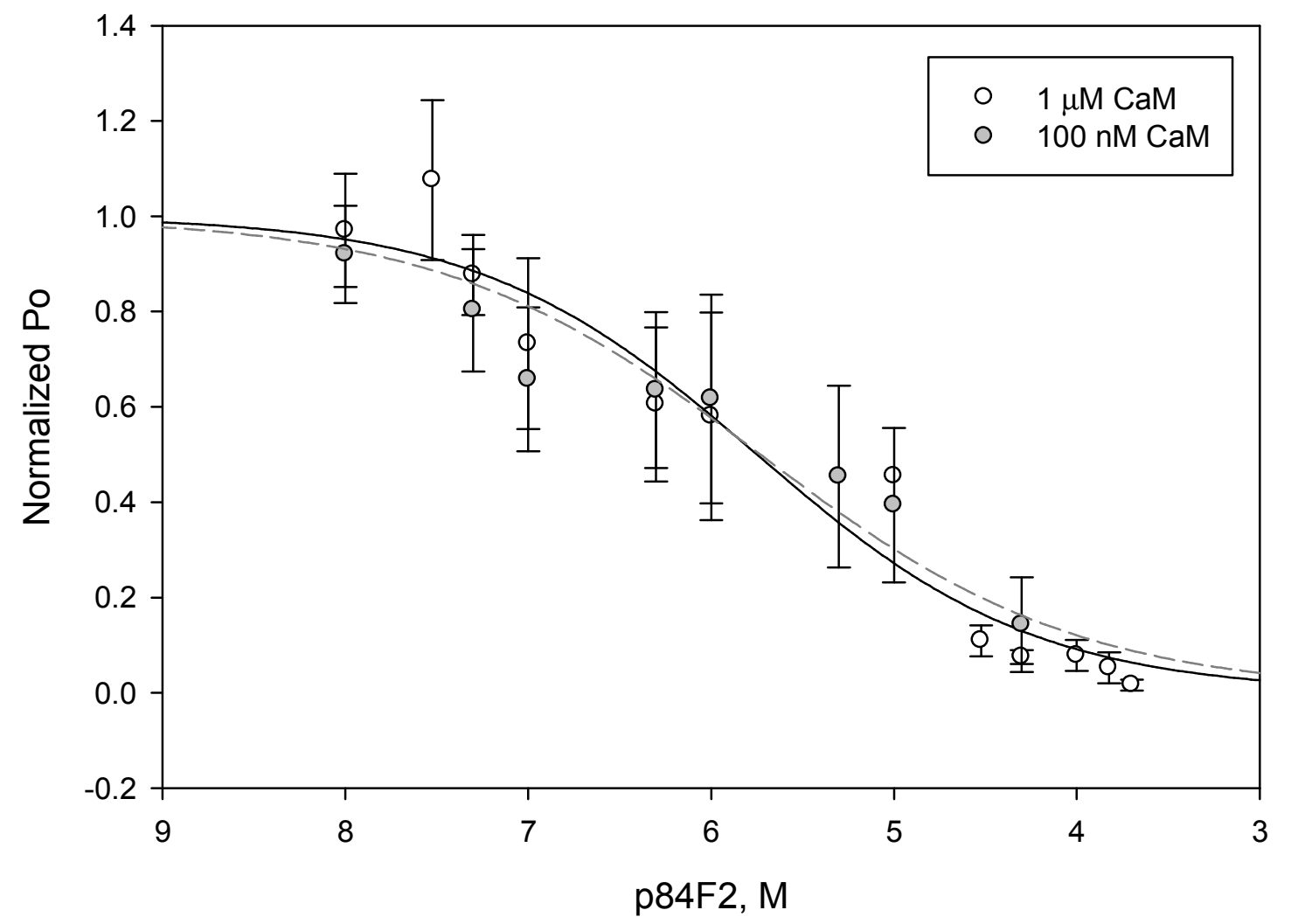

Figure 7.1 Inhibition of Purified RyR2 by 84F2 in the Presence of Calmodulin

$84 F 2$ has a reduced inhibition of purified RyR2 in the presence of calmodulin. Each data point normalized as the percent of the control $100 \mathrm{nM}$ (grey circles) or $1 \mu \mathrm{M}$ (open circles) calmodulin recording. Each data point represents 3-9 separate single channel recordings of at least two minutes. Fitting of the curve for $84 \mathrm{~F} 2$ in the presence of $1 \mu \mathrm{M} \mathrm{CaM}$ yields a $\mathrm{pIC}_{50}=5.75 \pm 0.39$ ( \pm standard error), $\mathrm{IC}_{50}=1.77 \mu \mathrm{M}, \mathrm{n}=3-9$. $84 \mathrm{~F} 2$ in the presence of $100 \mathrm{nM}$ CaM yields a pIC $\mathrm{C}_{50}=5.73 \pm 0.29$ ( \pm standard error), $\mathrm{IC}_{50}=1.86 \mu \mathrm{M}, \mathrm{n}=3-9$. 


\subsection{Calmodulin Inhibition of RyR2}

In addition to 84F2's inhibition of RyR2, CaM is also known to modulate channel activity. Single channel measurements of Po were examined prior to, and following, the addition of CaM in both the purified RyR2 and RyR2 from crude cardiac SR.

Table 7.1 summarizes CaM's effect on RyR2 Po. Data shows similar inhibition of purified RyR2 at both concentrations of CaM tested, with Po values decreasing to approximately $80 \%$ of control values. CaM showed no inhibition of RyR2 from crude cardiac SR channel activity.

\section{Table 7.1 Calmodulin Inhibition of RyR2}

\begin{tabular}{|l|l|l|l|l|}
\hline RyR2 Type & CaM $(\boldsymbol{\mu M})$ & Control Po & CaM Addition Po & $\mathbf{n}$ \\
\hline Purified & 0.1 & $0.96 \pm 0.17$ & $0.79 \pm 0.12$ & 9 \\
\hline Purified & 1 & $0.98 \pm 0.04$ & $0.80 \pm 0.09$ & 9 \\
\hline Non-Purified & 1 & $0.72 \pm 0.05$ & $0.76 \pm 0.14$ & 4 \\
\hline
\end{tabular}




\subsection{UV-Vis Spectrum of 84F2 and CaM}

84F2 and CaM's UV-Vis spectrums were examined to determine if interactions between CaM and 84F2 may exist.

Figure 7.2 shows the resulting UV-Vis spectrums of $84 \mathrm{~F} 2, \mathrm{CaM}$, as well as the combination of CaM and 84F2. A concentration of $1 \mu \mathrm{M} \mathrm{CaM}$ and was used to mimic the concentration used in single channel studies. CaM shows a strong absorption peak at $\sim 220 \mathrm{~nm} .3 \mu \mathrm{M}$ 84F2 resulted in a similarly sized absorption peak at $\sim 315 \mathrm{~nm}$. These two individual absorption spectrums were added together and plotted as the "calculated sum," shown on the graph. Absorption measurements made in the presence of both 3 $\mu \mathrm{M} 84 \mathrm{~F} 2$ and $1 \mu \mathrm{M}$ CaM resulted in an identical absorption spectrum as the calculated sum. 


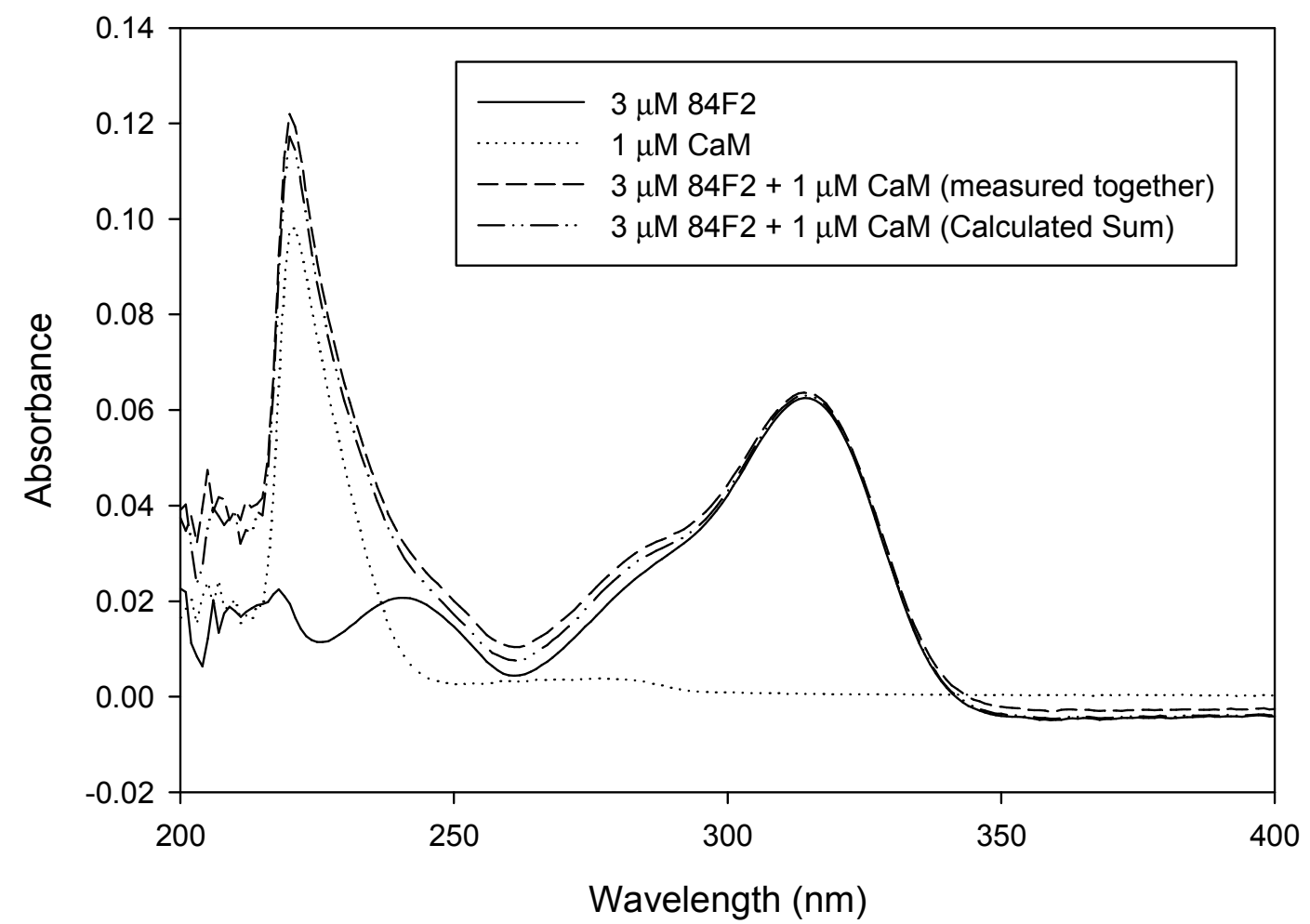

Figure 7.2 UV-Vis Spectrums of 84F2 and Calmodulin

Absorption spectrum measurements made in the presence of $84 \mathrm{~F} 2$ and CaM. $1 \mu \mathrm{M}$ CaM resulted in an absorption peak at $220 \mathrm{~nm}$. $3 \mu \mathrm{M} 84 \mathrm{~F} 2$ resulted in an absorption peak at $315 \mathrm{~nm}$. Individual spectrum measurements for the CaM and 84F2 were combined and plotted as the "calculated sum." Absorption measurements made in the presence of both CaM and 84F2 resulted in an identical spectrum to that of the "calculated sum." 


\subsection{Discussion: CaM Reduces 84F2's Potency in Inhibiting Purified RyR2}

Data from the RyR2 ${ }^{\mathrm{R} 176 \mathrm{O} /+}$ mouse model suggested that $84 \mathrm{~F} 2$ only inhibits a dysfunctional RyR2. Our single channel results supported this and indicate that this dysfunction is due to the absence of key regulatory proteins. These results along with the evidence in the literature all pointed towards CaM dissociation as a key factor in the ability of $84 F 2$ to inhibit RyR2 channel activity.

This hypothesis, which states that the absence of $\mathrm{CaM}$ is responsible for differences in 84F2's potency, was dependent on the presence, or absence, of CaM in our different RyR2 preparations used at the single channel level. The literature suggests that our preparation of purified RyR2 does not contain CaM. However, the presence of CaM in cardiac SR preparation in the bilayer is less certain. To test whether CaM was present in our two preparations, CaM modulation of single channel activity was measured.

CaM is known to modulate the activity of RyR2, acting as an inhibitor in its $\mathrm{Ca}^{2+}$-bound form $\left(\mathrm{Ca}^{2+}>1 \mu \mathrm{M}\right)$. Therefore, RyR2 activity at the single channel level following the addition of CaM to the cytoplasmic solution $\left(\mathrm{Ca}^{2+}=25 \mu \mathrm{M}\right)$, should indicate the presence or absence of native CaM.

Data, which is shown in Table 7.1, indicates a reduction in Po with the addition of CaM to purified RyR2. This $20 \%$ reduction in Po agrees with values in the literature for CaCaM inhibition of purified RyR2. The identical reduction in Po at both concentrations of CaM 
indicate that $100 \mathrm{nM}$ CaM is sufficient in saturating RyR2 binding sites. This is in strong support of the literature evidence that CaM is not present in our purified RyR2.

RyR2 from crude cardiac SR did not show a reduction in Po with the addition of saturating levels of CaM. In addition, control activity measurements of RyR2 from crude cardiac SR (Po $=0.72$ ) were lower than those for the purified RyR2 (Po $=0.98)$. RyR2 from crude cardiac SR control activity more closely resembled activity of purified RyR2 in the presence of CaM $(\mathrm{Po}=0.80)$. This indicates that CaM is likely still present in RyR2 from crude cardiac SR added to the bilayer.

The next question was whether the presence of CaM would decrease the inhibition of purified RyR2 by $84 \mathrm{~F} 2$. Figure 7.3 shows the dose-response curves for $84 \mathrm{~F} 2$ inhibition of purified RyR2 under identical conditions, with (grey circles) and without (black circles) 1 $\mu \mathrm{M}$ CaM present. The addition of $1 \mu \mathrm{M}$ CaM strongly reduced 84F2's potency. No overlap in the curves at any concentration of $84 \mathrm{~F} 2$ was observed until RyR2 was completely inhibited. The resulting half-maximal inhibition concentration increased from $8.13 \mathrm{nM}$ to $1.77 \mu \mathrm{M}$ with the addition of CaM. This result is in support of our hypothesis that CaM-dissociation from RyR2 is a key factor in 84F2's mechanism of inhibition.

One mechanism that could have accounted for this reduced inhibition of RyR2 by $84 \mathrm{~F} 2$, was an interaction between CaM and 84F2. The resulting in a CaM-84F2 complex may prevent 84F2 from inhibiting RyR2, effectively decreasing its potency. UV-Vis measurements (Figure 7.2) indicated no changes in absorption peaks for the CaM and 86 
$84 \mathrm{~F} 2$ when combined. This result indicated that no obvious interaction between the two existed, but did not rule out possible interactions that may not affect the absorption spectrums. However, the results showing that $84 \mathrm{~F} 2$ 's dose-response was nearly identical at 10-fold differences in CaM (Figure 7.1) indicates that a CaM-84F2 interaction is a highly unlikely as the mechanism of reduced inhibition of RyR2. 


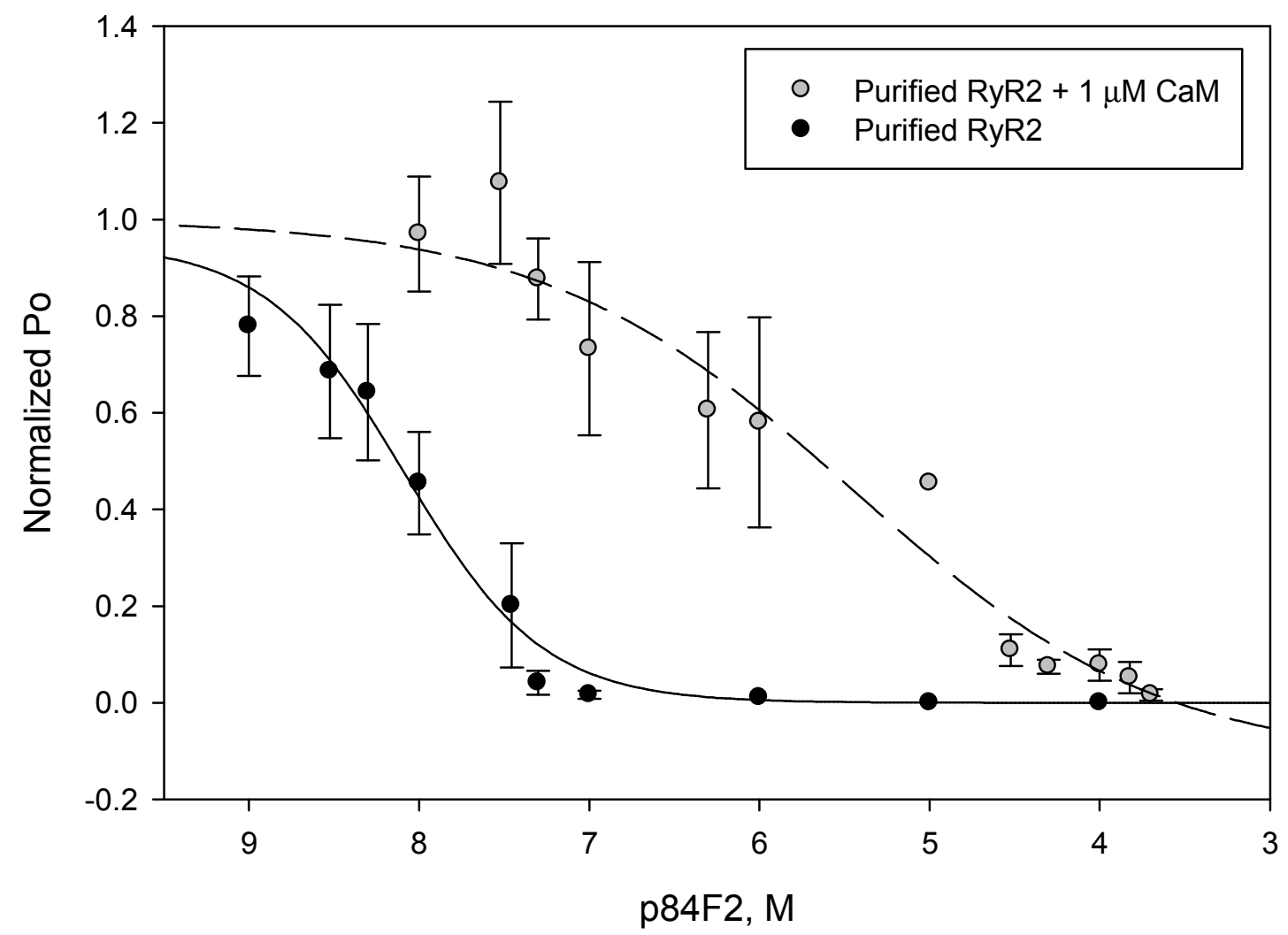

Figure 7.3 Calmodulin Decreases Inhibition of Purified RyR2 by 84F2

Dose-response curves for 84F2 inhibition of purified RyR2 purified RyR2 with (grey circles) and without (black circles) the addition of $1 \mu \mathrm{M}$ CaM. 84F2 a clear reduction in its strength of inhibition of purified RyR2 in the presence of $1 \mu \mathrm{M}$ CaM present. 84F2 in the absence of CaM resulted in an $\mathrm{IC}_{50}=8.13 \mathrm{nM}$. $84 \mathrm{~F} 2$ with the addition of $1 \mu \mathrm{M}$ CaM resulted in an $\mathrm{IC}_{50}=1.77 \mu \mathrm{M}$. 


\section{Conclusions and Model of 84F2's Mechanism}

Cardiac disorders resulting in improper $\mathrm{Ca}^{2+}$-cycling in cardiomyocytes can lead to life threatening conditions. $\mathrm{Ca}^{2+}$ leak from SR arises because of RyR2-dysfunction associated with these disorders. CPVT, is one such disorder in which mutations in RyR2 lead to its dysfunction during adrenergic stimulation. How adrenergic stimulation in CPVT leads to RyR2-dysfunction remains unclear. The literature suggests that phosphorylation of RyR2, during adrenergic stimulation, results in dissociation of key regulatory proteins ${ }^{86}$.

Here we have introduced a novel compound, 84F2, designed to inhibit RyR2-Ca ${ }^{2+}$ leak. Using a CPVT mouse model, RyR2 ${ }^{\mathrm{R} 1760 /+}$, we have shown $84 \mathrm{~F} 2$ to be effective in treating arrhythmogenic states that result from adrenergic stimulation, while not altering normal ECC. These are two ideal characteristics of a drug used to treat arrhythmias.

In addition to showing 84F2's efficacy in treating arrhythmia, we have investigated the underlying mechanisms involved in how 84F2 inhibits RyR2. The following key results were shown:

- 84F2 treats arrhythmia through direct RyR2 Inhibition. (Chapter 4)

- Following breakdown of 84F2, 78F3 can also inhibit RyR2 (Chapter 5). This is supported by data showing that 78F3 inhibits single channel activity and CaSPF in cells derived from a CPVT mouse model.

- $84 \mathrm{~F} 2$ 's inhibition of RyR2 is dependent on the absence of key regulating proteins. (Chapter 6)

- Inhibition of RyR2 by 84F2 is dependent on CaM-dissociation from RyR2. (Chapter 7) 
These findings supported the hypothesis in the literature which suggests that dissociation of regulatory proteins during adrenergic stimulation is the underlying mechanism of RyR2-dysfunction. We have further shown that the addition of one of these proteins, CaM, can prevent 84F2 from inhibiting purified RyR2.

Figure 8.1 shows three dose-response curves for $84 \mathrm{~F} 2$ inhibition of: purified RyR2 (black circles), RyR2 from crude cardiac SR (open circles), and purified RyR2 with $1 \mu \mathrm{M}$ CaM (grey circles). The RyR2 from crude cardiac SR is only inhibited at high concentrations of 84F2. Purified RyR2 was potently inhibited by 84F2 at similar concentrations as those seen in the CPVT model. The addition of $1 \mu \mathrm{M}$ CaM to the purified RyR2, shifted 84F2's inhibition to concentrations like those seen in RyR2 from crude cardiac SR.

These results, as summarized in Table 8.1, support the theory that CaM-dissociation is an underlying cause of RyR2-dysfunction in CPVT.

Table 8.1 Summary of 84F2 Inhibition of RyR2

\begin{tabular}{|l|l|l|}
\hline Assay & RyR2 & IC50 (nM) \\
\hline Single Channel & Purified & 8.21 \\
\hline CaSPF & RyR2 ${ }^{\mathrm{R} 176 \mathrm{Q} /+}$ & 35.40 \\
\hline Single Channel & Crude Cardiac SR & 8,110 \\
\hline Single Channel & Purified $+1 \mu \mathrm{M}$ CaM & 1,770 \\
\hline
\end{tabular}




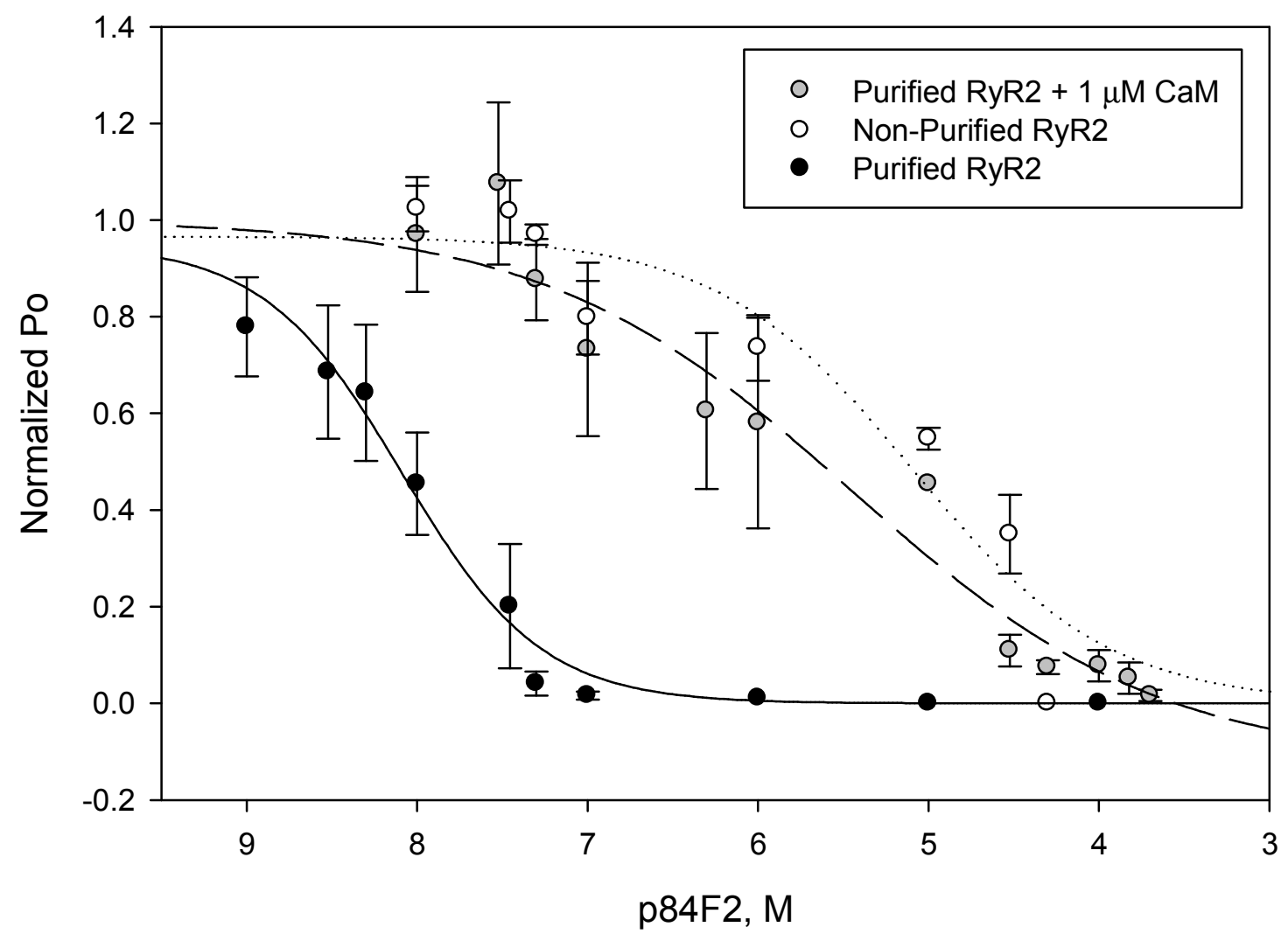

Figure 8.1 Comparison of RyR2 Single Channel Activity by 84F2

84F2 showed weak inhibition of RyR2 from crude cardiac SR (Open Circles). 84F2 potently inhibited purified RyR2 (black circles). Addition of $1 \mu \mathrm{M}$ CaM decreased 84F2's inhibition of purified RyR2 (grey circles). All experiments carried out under identical conditions, as described in the methods. 
Based on our findings we propose the following model, underlying the mechanism of RyR2 ${ }^{\mathrm{R} 176 \mathrm{q} /+}$ arrhythmia and 84F2's targeted treatment.

- Adrenergic stimulation of RyR2 ${ }^{\mathrm{R} 1760 /+}$ cells results in the dissociation of CaM.

- $\mathrm{RyR2}^{\mathrm{R} 176 \mathrm{O} /+}$, deficient in CaM, becomes leaky to $\mathrm{Ca}^{2+}$, which leads to arrhythmias.

- $84 \mathrm{~F} 2$ then binds to, and stabilizes the receptor, decreases the $\mathrm{Ca}^{2+}$ leak, and arrhythmias.

- In the absence of adrenergic stimulation, RyR2 ${ }^{\mathrm{R} 1760 /+}$ is still associated with CaM.

- CaM stabilizes RyR2 and prevents 84F2 from inhibiting ECC. 


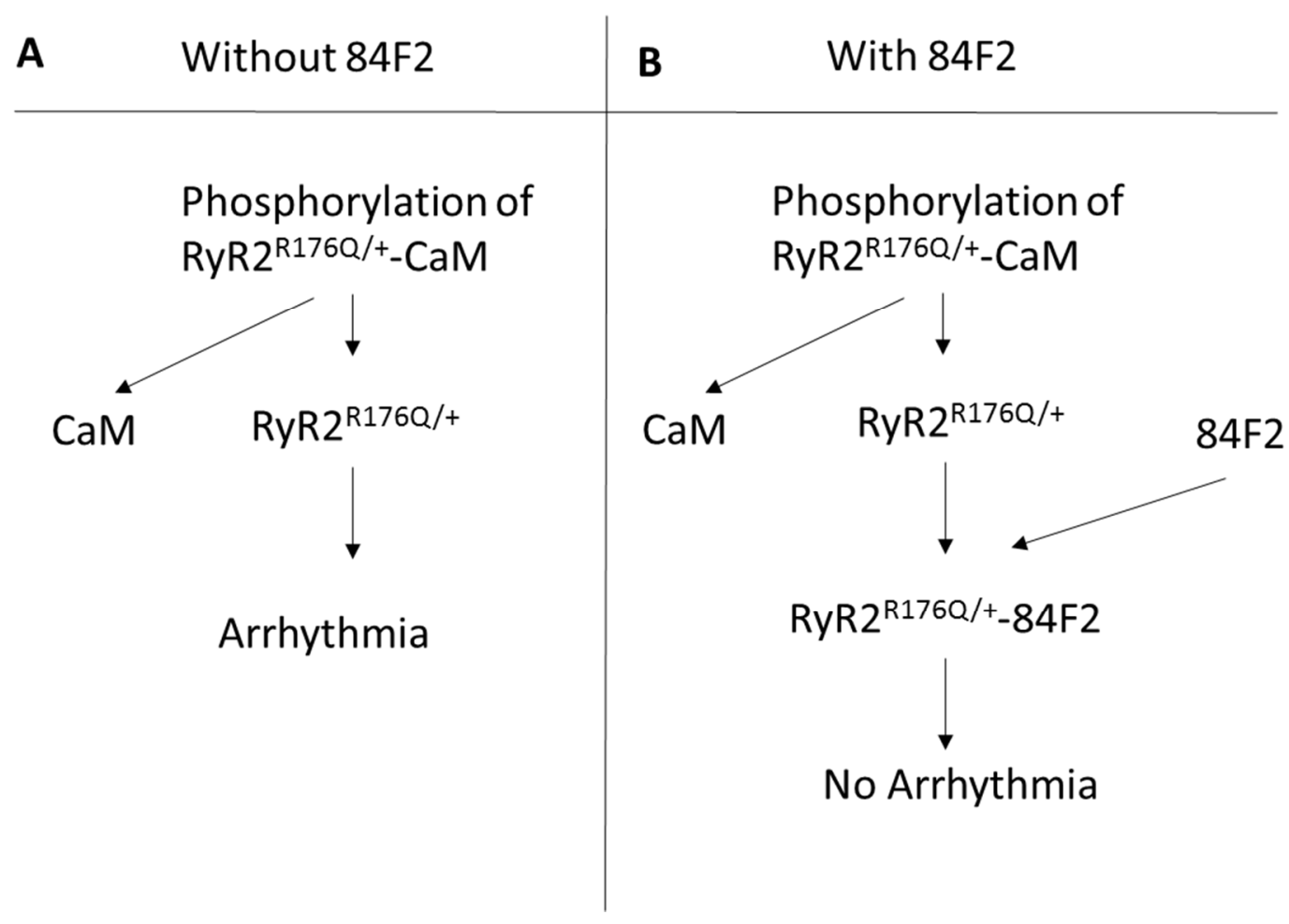

Figure 8.2 Model of 84F2 Inhibition of Arrhythmia resulting from RyR2 ${ }^{R 176 Q /+}$

A) RyR2 ${ }^{\mathrm{R} 1760 /+}$ loses their associated CaM following adrenergic stimulation. This can lead to arrhythmia. B) 84F2 stabilizes RyR2 ${ }^{\mathrm{R} 1760 /+}$ following the dissociation of CaM. This diminishes the arrhythmia. 


\section{References}

${ }^{1}$ Don W. Fawcett and N. Scott McNutt, "The Ultrastructure of the Cat Myocardium," The Journal of Cell Biology 42, no. 1 (July 1, 1969): 1-45, doi:10.1083/jcb.42.1.1.

${ }^{2}$ T. Imagawa et al., "Purified Ryanodine Receptor from Skeletal Muscle Sarcoplasmic Reticulum Is the Ca2+-Permeable Pore of the Calcium Release Channel.," Journal of Biological Chemistry 262, no. 34 (December 5, 1987): 16636-43.

3 I. I. Serysheva et al., "Electron Cryomicroscopy and Angular Reconstitution Used to Visualize the Skeletal Muscle Calcium Release Channel," Nature Structural Biology 2, no. 1 (January 1995): 18-24.

${ }^{4}$ H. Cheng, W. J. Lederer, and M. B. Cannell, "Calcium Sparks: Elementary Events Underlying Excitation-Contraction Coupling in Heart Muscle," Science (New York, N.Y.) 262, no. 5134 (October 29, 1993): 740-44.

${ }^{5}$ M B Cannell, H Cheng, and W J Lederer, "Spatial Non-Uniformities in [Ca2+]i during Excitation-Contraction Coupling in Cardiac Myocytes.," Biophysical Journal 67, no. 5 (November 1994): 1942-56.

${ }^{6}$ T. Jayaraman et al., "FK506 Binding Protein Associated with the Calcium Release Channel (Ryanodine Receptor).," Journal of Biological Chemistry 267, no. 14 (May 15, 1992): 9474-77.

${ }^{7}$ Masafumi Yano et al., "FKBP12.6-Mediated Stabilization of Calcium-Release Channel (Ryanodine Receptor) as a Novel Therapeutic Strategy Against Heart Failure," Circulation 107, no. 3 (January 28, 2003): 6, doi:10.1161/01.CIR.0000044917.74408.BE.

8 Tao Guo et al., "Kinetics of FKBP12.6 Binding to Ryanodine Receptors in Permeabilized Cardiac Myocytes and Effects on Ca Sparks," Circulation Research 106, no. 11 (June 11, 2010): 12, doi:10.1161/CIRCRESAHA.110.219816.

${ }^{9}$ David H. MacLennan and P. T. S. Wong, "Isolation of a Calcium-Sequestering Protein from Sarcoplasmic Reticulum," Proceedings of the National Academy of Sciences 68, no. 6 (June 1, 1971): 1231-35.

${ }^{10}$ Lin Zhang et al., "Complex Formation between Junctin, Triadin, Calsequestrin, and the Ryanodine Receptor PROTEINS OF THE CARDIAC JUNCTIONAL SARCOPLASMIC RETICULUM MEMBRANE," Journal of Biological Chemistry 272, no. 37 (September 12, 1997): 23389-97, doi:10.1074/jbc.272.37.23389.

11 S. O. Marx et al., "PKA Phosphorylation Dissociates FKBP12.6 from the Calcium Release Channel (Ryanodine Receptor): Defective Regulation in Failing Hearts," Cell 101, no. 4 (May 12, 2000): 365-76.

${ }^{12}$ Xander H. T. Wehrens et al., "Ca2+/calmodulin-Dependent Protein Kinase II Phosphorylation Regulates the Cardiac Ryanodine Receptor," Circulation Research 94, no. 6 (April 2, 2004): e61-70, doi:10.1161/01.RES.0000125626.33738.E2.

${ }^{13}$ A. J. Lokuta et al., "Modulation of Cardiac Ryanodine Receptors of Swine and Rabbit by a Phosphorylation-Dephosphorylation Mechanism," The Journal of Physiology 487 ( Pt 3) (September 15, 1995): 609-22. 
${ }^{14}$ Steven O. Marx et al., "Phosphorylation-Dependent Regulation of Ryanodine Receptors," The Journal of Cell Biology 153, no. 4 (May 14, 2001): 699-708.

${ }^{15} \mathrm{C}$. Callaway et al., "Localization of the High and Low Affinity [3H]ryanodine Binding Sites on the Skeletal Muscle Ca2+ Release Channel," The Journal of Biological Chemistry 269, no. 22 (June 3, 1994): 15876-84.

${ }^{16} \mathrm{~A}$. Chu et al., "Ryanodine as a Probe for the Functional State of the Skeletal Muscle Sarcoplasmic Reticulum Calcium Release Channel," Molecular Pharmacology 37, no. 5 (May 1990): 735-41.

${ }^{17}$ R Sitsapesan and A J Williams, "Mechanisms of Caffeine Activation of Single CalciumRelease Channels of Sheep Cardiac Sarcoplasmic Reticulum.," The Journal of Physiology 423 (April 1990): 425-39.

${ }^{18}$ A. B. Brillantes et al., "Stabilization of Calcium Release Channel (Ryanodine Receptor) Function by FK506-Binding Protein," Cell 77, no. 4 (May 20, 1994): 513-23.

${ }^{19} \mathrm{G}$. Meissner, E. Darling, and J. Eveleth, "Kinetics of Rapid Ca2+ Release by Sarcoplasmic Reticulum. Effects of $\mathrm{Ca} 2+, \mathrm{Mg} 2+$, and Adenine Nucleotides," Biochemistry 25, no. 1 (January 14, 1986): 236-44.

20 Ibid.

${ }^{21}$ D. R. Laver et al., "Cytoplasmic Ca2+ Inhibits the Ryanodine Receptor from Cardiac Muscle," The Journal of Membrane Biology 147, no. 1 (n.d.): 7-22, doi:10.1007/BF00235394.

${ }^{22}$ S. Györke, V. Lukyanenko, and I. Györke, "Dual Effects of Tetracaine on Spontaneous Calcium Release in Rat Ventricular Myocytes," The Journal of Physiology 500 ( Pt 2) (April 15, 1997): 297-309.

${ }^{23}$ Steven M Pogwizd and Donald M Bers, "Cellular Basis of Triggered Arrhythmias in Heart Failure," Trends in Cardiovascular Medicine 14, no. 2 (February 2004): 61-66, doi:10.1016/j.tcm.2003.12.002.

${ }^{24}$ Steven M. Pogwizd et al., "Upregulation of $\mathrm{Na}+\mathrm{Ca} 2+$ Exchanger Expression and Function in an Arrhythmogenic Rabbit Model of Heart Failure," Circulation Research 85, no. 11 (November 26, 1999): 1009-19, doi:10.1161/01.RES.85.11.1009.

${ }^{25}$ Donald M. Bers, David A. Eisner, and Héctor H. Valdivia, "Sarcoplasmic Reticulum Ca2+ and Heart Failure," Circulation Research 93, no. 6 (September 19, 2003): 487-90, doi:10.1161/01.RES.0000091871.54907.6B.

${ }^{26}$ Meiso Hayashi et al., "Incidence and Risk Factors of Arrhythmic Events in Catecholaminergic Polymorphic Ventricular Tachycardia," Circulation 119, no. 18 (May 12, 2009): 2426-34, doi:10.1161/CIRCULATIONAHA.108.829267.

${ }^{27}$ Hadas Lahat et al., "A Missense Mutation in a Highly Conserved Region of CASQ2 Is Associated with Autosomal Recessive Catecholamine-Induced Polymorphic Ventricular Tachycardia in Bedouin Families from Israel," American Journal of Human Genetics 69, no. 6 (December 2001): 1378-84. 
${ }^{28}$ Mette Nyegaard et al., "Mutations in Calmodulin Cause Ventricular Tachycardia and Sudden Cardiac Death," The American Journal of Human Genetics 91, no. 4 (October 5, 2012): 703-12, doi:10.1016/j.ajhg.2012.08.015.

${ }^{29}$ Päivi J. Laitinen et al., "Mutations of the Cardiac Ryanodine Receptor (RyR2) Gene in Familial Polymorphic Ventricular Tachycardia," Circulation 103, no. 4 (January 30, 2001): 485-90, doi:10.1161/01.CIR.103.4.485.

30 Silvia G. Priori and S. R. Wayne Chen, "Inherited Dysfunction of Sarcoplasmic Reticulum Ca2+ Handling and Arrhythmogenesis," Circulation Research 108, no. 7 (April 1, 2011): 871-83, doi:10.1161/CIRCRESAHA.110.226845.

${ }^{31}$ Masafumi Yano et al., "Abnormal Ryanodine Receptor Function in Heart Failure," Pharmacology \& Therapeutics 107, no. 3 (September 2005): 377-91, doi:10.1016/j.pharmthera.2005.04.003.

32 Marx et al., "Phosphorylation-Dependent Regulation of Ryanodine Receptors."

${ }^{33}$ Hayashi et al., "Incidence and Risk Factors of Arrhythmic Events in Catecholaminergic Polymorphic Ventricular Tachycardia."

${ }^{34}$ Nian Liu et al., "Abnormal Propagation of Calcium Waves and Ultrastructural Remodeling in Recessive Catecholaminergic Polymorphic Ventricular TachycardiaNovelty and Significance," Circulation Research 113, no. 2 (July 5, 2013): 142-52, doi:10.1161/CIRCRESAHA.113.301783.

${ }^{35}$ Christian van der Werf et al., "Flecainide Therapy Reduces Exercise-Induced Ventricular Arrhythmias in Patients With Catecholaminergic Polymorphic Ventricular Tachycardia," Journal of the American College of Cardiology 57, no. 22 (May 31, 2011): 2244-54, doi:10.1016/j.jacc.2011.01.026.

${ }^{36}$ Yano et al., "FKBP12.6-Mediated Stabilization of Calcium-Release Channel (Ryanodine Receptor) as a Novel Therapeutic Strategy Against Heart Failure."

${ }^{37}$ Stephan E. Lehnart et al., "Leaky Ca2+ Release Channel/ryanodine Receptor 2 Causes Seizures and Sudden Cardiac Death in Mice," The Journal of Clinical Investigation 118, no. 6 (June 2008): 2230-45, doi:10.1172/JCl35346.

${ }^{38}$ Xander H. T. Wehrens et al., "FKBP12.6 Deficiency and Defective Calcium Release Channel (Ryanodine Receptor) Function Linked to Exercise-Induced Sudden Cardiac Death," Cell 113, no. 7 (June 27, 2003): 829-40, doi:10.1016/S0092-8674(03)00434-3. ${ }^{39}$ Guo et al., "Kinetics of FKBP12.6 Binding to Ryanodine Receptors in Permeabilized Cardiac Myocytes and Effects on Ca Sparks."

${ }^{40}$ Natascia Tiso et al., "The Binding of the RyR2 Calcium Channel to Its Gating Protein FKBP12.6 Is Oppositely Affected by ARVD2 and VTSIP Mutations," Biochemical and Biophysical Research Communications 299, no. 4 (December 13, 2002): 594-98, doi:10.1016/S0006-291X(02)02689-X.

${ }^{41}$ Dawei Jiang et al., "Enhanced Store Overload-Induced Ca2+ Release and Channel Sensitivity to Luminal Ca2+ Activation Are Common Defects of RyR2 Mutations Linked to Ventricular Tachycardia and Sudden Death," Circulation Research 97, no. 11 (November 25, 2005): 1173-81, doi:10.1161/01.RES.0000192146.85173.4b. 
${ }^{42}$ Christopher H. George, Gemma V. Higgs, and F. Anthony Lai, "Ryanodine Receptor Mutations Associated With Stress-Induced Ventricular Tachycardia Mediate Increased Calcium Release in Stimulated Cardiomyocytes," Circulation Research 93, no. 6 (September 19, 2003): 531-40, doi:10.1161/01.RES.0000091335.07574.86.

${ }^{43}$ Le Xu et al., "Activation of the Cardiac Calcium Release Channel (Ryanodine Receptor) by Poly-S-Nitrosylation," Science 279, no. 5348 (January 9, 1998): 234-37, doi:10.1126/science.279.5348.234.

${ }^{44}$ Jonathan J. Abramson and Guy Salama, "Critical Sulfhydryls Regulate Calcium Release from Sarcoplasmic Reticulum," Journal of Bioenergetics and Biomembranes 21, no. 2 (n.d.): 283-94, doi:10.1007/BF00812073.

${ }^{45}$ Noriaki lkemoto and Takeshi Yamamoto, "Regulation of Calcium Release by Interdomain Interaction within Ryanodine Receptors," Frontiers in Bioscience: A Journal and Virtual Library 7 (March 1, 2002): d671-683.

${ }^{46}$ Tetsuro Oda et al., "In Cardiomyocytes, Binding of Unzipping Peptide Activates Ryanodine Receptor 2 and Reciprocally Inhibits Calmodulin Binding," Circulation Research 112, no. 3 (February 1, 2013): 487-97, doi:10.1161/CIRCRESAHA.111.300290.

${ }^{47}$ Naohiro Yamaguchi et al., "Early Cardiac Hypertrophy in Mice with Impaired Calmodulin Regulation of Cardiac Muscle Ca2+ Release Channel," Journal of Clinical Investigation 117, no. 5 (May 1, 2007): 1344-53, doi:10.1172/JCI29515.

${ }^{48}$ Y. Sudhakar Babu et al., "Three-Dimensional Structure of Calmodulin," Nature 315, no. 6014 (May 2, 1985): 37-40, doi:10.1038/315037a0.

${ }^{49}$ Mingjie Zhang, Toshiyuki Tanaka, and Mitsuhiko Ikura, "Calcium-Induced Conformational Transition Revealed by the Solution Structure of Apo Calmodulin," Nature Structural \& Molecular Biology 2, no. 9 (September 1995): 758-67, doi:10.1038/nsb0995-758.

${ }^{50}$ Lars S. Maier et al., "Dynamic Changes in Free Ca-Calmodulin Levels in Adult Cardiac Myocytes," Journal of Molecular and Cellular Cardiology 41, no. 3 (September 2006): 451-58, doi:10.1016/j.yjmcc.2006.04.020.

${ }^{51}$ Shiro Kakiuchi et al., "Quantitative Determinations of Calmodulin in the Supernatant and Particulate Fractions of Mammalian Tissues," Journal of Biochemistry 92, no. 4 (October 1, 1982): 1041-48.

${ }^{52} \mathrm{Xu}$ Wu and Donald M. Bers, "Free and Bound Intracellular Calmodulin Measurements in Cardiac Myocytes," Cell Calcium 41, no. 4 (April 2007): 353-64, doi:10.1016/j.ceca.2006.07.011.

${ }^{53} \mathrm{C}$. Ban et al., "Structure of the Recombinant Paramecium Tetraurelia Calmodulin at 1.68 A Resolution," Acta Crystallographica. Section D, Biological Crystallography 50, no. Pt 1 (January 1, 1994): 50-63, doi:10.1107/S09074444993007991.

${ }^{54}$ A Tripathy et al., "Calmodulin Activation and Inhibition of Skeletal Muscle Ca2+ Release Channel (Ryanodine Receptor).," Biophysical Journal 69, no. 1 (July 1995): 10619. 
${ }^{55}$ David M. Balshaw et al., "Calmodulin Binding and Inhibition of Cardiac Muscle Calcium Release Channel (Ryanodine Receptor)," Journal of Biological Chemistry 276, no. 23 (June 8, 2001): 20144-53, doi:10.1074/jbc.M010771200.

${ }^{56} \mathrm{~T}$. Ikemoto et al., "Effect of Calmodulin on Ca2+-Induced Ca2+ Release of Skeletal Muscle from Mutant Mice Expressing Either Ryanodine Receptor Type 1 or Type 3," Pflügers Archiv 437, no. 1 (n.d.): 43-48, doi:10.1007/s004240050744.

${ }^{57}$ Catherine Porter Moore et al., "Apocalmodulin and Ca2+Calmodulin Bind to the Same Region on the Skeletal Muscle Ca2+ Release Channel," Biochemistry 38, no. 26 (June 1, 1999): 8532-37, doi:10.1021/bi9907431.

${ }^{58}$ George G. Rodney et al., "Calcium Binding to Calmodulin Leads to an N-Terminal Shift in Its Binding Site on the Ryanodine Receptor," Journal of Biological Chemistry 276, no. 3 (January 19, 2001): 2069-74, doi:10.1074/jbc.M008891200.

${ }^{59}$ Moore et al., "Apocalmodulin and Ca2+ Calmodulin Bind to the Same Region on the Skeletal Muscle Ca2+ Release Channel."

${ }^{60}$ Naohiro Yamaguchi et al., "Molecular Basis of Calmodulin Binding to Cardiac Muscle Ca2+ Release Channel (Ryanodine Receptor)," Journal of Biological Chemistry 278, no. 26 (June 27, 2003): 23480-86, doi:10.1074/jbc.M301125200.

${ }^{61}$ Balshaw et al., "Calmodulin Binding and Inhibition of Cardiac Muscle Calcium Release Channel (Ryanodine Receptor)."

${ }^{62}$ Ikemoto et al., "Effect of Calmodulin on Ca2+-Induced Ca2+ Release of Skeletal Muscle from Mutant Mice Expressing Either Ryanodine Receptor Type 1 or Type 3."

${ }^{63}$ Tripathy et al., "Calmodulin Activation and Inhibition of Skeletal Muscle Ca2+ Release Channel (Ryanodine Receptor)."

${ }^{64} \mathrm{G}$. Meissner and J. S. Henderson, "Rapid Calcium Release from Cardiac Sarcoplasmic Reticulum Vesicles Is Dependent on $\mathrm{Ca} 2+$ and Is Modulated by Mg2+, Adenine Nucleotide, and Calmodulin," The Journal of Biological Chemistry 262, no. 7 (March 5, 1987): 3065-73.

${ }^{65}$ Bradley R. Fruen et al., "Differential Ca2+ Sensitivity of Skeletal and Cardiac Muscle Ryanodine Receptors in the Presence of Calmodulin," American Journal of Physiology Cell Physiology 279, no. 3 (September 1, 2000): C724-33.

${ }^{66}$ Le Xu and Gerhard Meissner, "Mechanism of Calmodulin Inhibition of Cardiac Sarcoplasmic Reticulum Ca2+ Release Channel (Ryanodine Receptor)," Biophysical Journal 86, no. 2 (February 2004): 797-804, doi:10.1016/S0006-3495(04)74155-7. ${ }^{67}$ Balshaw et al., "Calmodulin Binding and Inhibition of Cardiac Muscle Calcium Release Channel (Ryanodine Receptor)."

${ }^{68}$ Meissner and Henderson, "Rapid Calcium Release from Cardiac Sarcoplasmic Reticulum Vesicles Is Dependent on Ca2+ and Is Modulated by Mg2+, Adenine Nucleotide, and Calmodulin."

${ }^{69}$ Yamaguchi et al., "Early Cardiac Hypertrophy in Mice with Impaired Calmodulin Regulation of Cardiac Muscle Ca2+ Release Channel." 
70 Makoto Ono et al., "Dissociation of Calmodulin from Cardiac Ryanodine Receptor Causes Aberrant $\mathrm{Ca}(2+)$ Release in Heart Failure," Cardiovascular Research 87, no. 4 (September 1, 2010): 609-17, doi:10.1093/cvr/cvq108.

${ }^{71}$ Xiaojuan Xu et al., "Defective Calmodulin Binding to the Cardiac Ryanodine Receptor Plays a Key Role in CPVT-Associated Channel Dysfunction," Biochemical and Biophysical Research Communications 394, no. 3 (April 9, 2010): 660-66, doi:10.1016/j.bbrc.2010.03.046.

72 Xun Ai et al., "Ca2+/Calmodulin-Dependent Protein Kinase Modulates Cardiac Ryanodine Receptor Phosphorylation and Sarcoplasmic Reticulum Ca2+ Leak in Heart Failure," Circulation Research 97, no. 12 (December 9, 2005): 1314-22, doi:10.1161/01.RES.0000194329.41863.89.

${ }^{73}$ Yi Yang et al., "Cardiac Myocyte Z-Line Calmodulin Is Mainly RyR2-Bound, and Reduction Is Arrhythmogenic and Occurs in Heart FailureNovelty and Significance," Circulation Research 114, no. 2 (January 17, 2014): 295-306, doi:10.1161/CIRCRESAHA.114.302857.

74 Oda et al., "In Cardiomyocytes, Binding of Unzipping Peptide Activates Ryanodine Receptor 2 and Reciprocally Inhibits Calmodulin Binding."

${ }^{75} \mathrm{R}$ Sitsapesan and A J Williams, "Mechanisms of Caffeine Activation of Single CalciumRelease Channels of Sheep Cardiac Sarcoplasmic Reticulum.," The Journal of Physiology 423 (April 1990): 425-39.

${ }^{76}$ H. M. Kalckar, "Differential Spectrophotometry of Purine Compounds by Means of Specific Enzymes; Studies of the Enzymes of Purine Metabolism," The Journal of Biological Chemistry 167, no. 2 (February 1947): 461-75.

${ }^{77}$ F. Anthony Lai et al., "Purification and Reconstitution of the Calcium Release Channel from Skeletal Muscle," Nature 331, no. 6154 (January 28, 1988): 315-19, doi:10.1038/331315a0.

78 Paul Mueller et al., "Reconstitution of Cell Membrane Structure in Vitro and Its Transformation into an Excitable System," Nature 194 (June 1, 1962): 979-80, doi:10.1038/194979a0.

${ }^{79}$ Laver et al., "Cytoplasmic Ca2+ Inhibits the Ryanodine Receptor from Cardiac Muscle." ${ }^{80}$ F. J. Sigworth and S. M. Sine, "Data Transformations for Improved Display and Fitting of Single-Channel Dwell Time Histograms," Biophysical Journal 52, no. 6 (December 1, 1987): 1047-54, doi:10.1016/S0006-3495(87)83298-8.

81 Benjamin S. Marinov et al., "Non-Thiol Reagents Regulate Ryanodine Receptor Function by Redox Interactions That Modify Reactive Thiols," Antioxidants \& Redox Signaling 9, no. 5 (May 2007): 609-21, doi:10.1089/ars.2006.1426.

82 Masafumi Yano, "Ryanodine Receptor as a New Therapeutic Target of Heart Failure and Lethal Arrhythmia," ResearchGate 72, no. 4 (May 1, 2008): 509-14, doi:10.1253/circj.72.509. 83 Ono et al., "Dissociation of Calmodulin from Cardiac Ryanodine Receptor Causes Aberrant $\mathrm{Ca}(2+)$ Release in Heart Failure." 
${ }^{84}$ Yamaguchi et al., "Early Cardiac Hypertrophy in Mice with Impaired Calmodulin Regulation of Cardiac Muscle Ca2+ Release Channel."

${ }^{85}$ Xu et al., "Defective Calmodulin Binding to the Cardiac Ryanodine Receptor Plays a Key Role in CPVT-Associated Channel Dysfunction."

86 Ibid. 